\title{
Rates of compact object coalescences
}

\author{
Ilya Mandel ${ }^{1,2,3}$ (D) Floor S. Broekgaarden ${ }^{4}$
}

Received: 13 July 2021 / Accepted: 6 December 2021 / Published online: 17 February 2022

(C) The Author(s) 2022

\begin{abstract}
Gravitational-wave detections are enabling measurements of the rate of coalescences of binaries composed of two compact objects-neutron stars and/or black holes. The coalescence rate of binaries containing neutron stars is further constrained by electromagnetic observations, including Galactic radio binary pulsars and short gamma-ray bursts. Meanwhile, increasingly sophisticated models of compact objects merging through a variety of evolutionary channels produce a range of theoretically predicted rates. Rapid improvements in instrument sensitivity, along with plans for new and improved surveys, make this an opportune time to summarise the existing observational and theoretical knowledge of compact-binary coalescence rates.
\end{abstract}

Keywords Black holes · Neutron stars · Stellar binaries · Gravitational waves

\section{Contents}

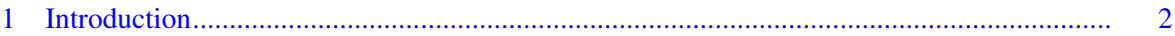

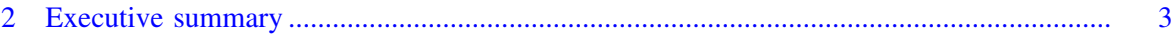

2.1 Gravitational waves .................................................................................... 13

Ilya Mandel

ilya.mandel@monash.edu

Floor S. Broekgaarden

floor.broekgaarden@cfa.harvard.edu

1 Monash Centre for Astrophysics, School of Physics and Astronomy, Monash University, Clayton, VIC 3800, Australia

2 OzGrav, ARC Centre of Excellence for Gravitational Wave Discovery, Hawthorn, Australia

3 Institute of Gravitational Wave Astronomy and School of Physics and Astronomy, University of Birmingham, Birmingham B15 2TT, UK

4 Institute for Theory and Computation (ITC), Center for Astrophysics I Harvard \& Smithsonian, 60 Garden St., Cambridge, MA 02138, USA 
2.2 Short gamma-ray bursts and kilonovae .................................................................... 14

2.3 Pulsars in Galactic compact object binaries........................................................... 15

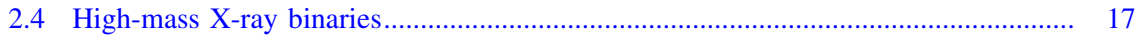

2.5 Other observational constraints ............................................................................... 17

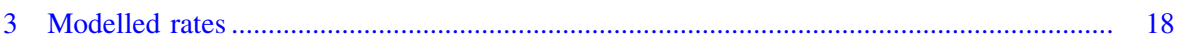

3.1 Isolated binary evolution modelling (population synthesis) ................................... 19

3.2 Dynamical formation ............................................................................................ 24

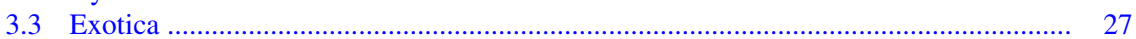

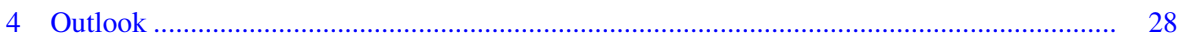

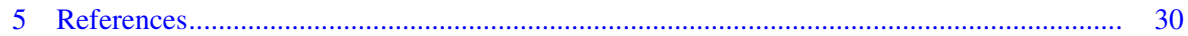

\section{Introduction}

It has been more than a decade since Abadie (2010) reviewed the literature on compact-object merger rate predictions. At that time, a handful of ultimately merging double neutron star (NS) systems in the Milky Way Galaxy were known from radio pulsar observations, starting with the discovery of Hulse and Taylor (1975). It was believed that short gamma-ray bursts (SGRBs) were associated with mergers of two NSs. But no actual compact object mergers were definitively observed. Therefore, most of the rate predictions, particularly for mergers involving black holes (BHs), were based purely on theoretical models (see, e.g., Mandel and O'Shaughnessy 2010 for a review of the status of models at that time).

The intervening decade has completely changed this landscape. On 14 September, 2015, the advanced Laser Interferometer Gravitational-wave Observatory (LIGO, Aasi et al. 2015) detected the first chirp of gravitational waves from a binary BH merger, GW150914 (Abbott et al. 2016b). Since then, nine more binary $\mathrm{BH}$ coalescences were observed during the first and second observing runs of advanced LIGO and Virgo detectors (Abbott et al. 2019b). The data release from the third observing run brought the total number of confidently detected binary $\mathrm{BH}$ coalescences to approximately 70 (Abbott et al. 2021d). The first detection of a binary neutron star merger, GW170817 (Abbott et al. 2017b), was accompanied by a short GRB (Abbott et al. 2017a) and a kilonova (Abbott et al. 2017c), firmly establishing the connection between these phenomena. A second confident binary neutron star detection, GW190425 (Abbott et al. 2020a), followed, along with detections of two NS-BH mergers, GW200105 and GW200115 (Abbott et al. 2021a).

At the same time, significant progress has been made in the theoretical modelling of the sources of compact binary coalescences. This has included more detailed exploration of the classical channel of isolated binary evolution, typically through the common-envelope phase (van den Heuvel 1976; Smarr and Blandford 1976; Tutukov and Yungelson 1993) (or possibly through stable mass transfer alone, van den Heuvel et al. 2017; Inayoshi et al. 2017), along with significant new developments in the investigation of alternative channels, including dynamical interaction in dense stellar environments (Sigurdsson and Hernquist 1993; Kulkarni et al. 1993; Portegies Zwart and McMillan 2000); mergers in binaries of rapidly 
rotating stars undergoing chemically homogeneous evolution (Mandel and de Mink 2016; Marchant et al. 2016); mergers facilitated by the Lidov-Kozai resonance (Lidov 1962; Kozai 1962) in hierarchical triple systems; and even mergers of primordial black holes of cosmological rather than astrophysical origin (Bird et al. 2016; Ali-Haïmoud et al. 2017).

The coupled advances in observations and theory are making it possible to quantitatively compare rate predictions against observations for the first time (e.g., Abbott et al. 2019a, 2021e; Belczynski et al. 2018a; Wysocki et al. 2018; Neijssel et al. 2019; Belczynski et al. 2020; Farmer et al. 2020; Bavera et al. 2021). Beyond this, they provide critical input into understanding the chemical enrichment of the Universe, particularly with elements created through r-process nucleosynthesis likely associated with mergers involving NSs with their neutron-rich material (Kasen et al. 2017; Côté et al. 2018; Metzger 2019; Kobayashi et al. 2020). The merger rates determine prospects for future Earth-based and space-borne gravitational-wave detectors, and will impact detector design (Amaro-Seoane et al. 2017; Adhikari et al. 2019; Reitze et al. 2019). Merger rates feed into constraints or predictions on other observables, ranging from kilonovae associated with binary NS mergers (e.g., Kasliwal et al. 2020; Mochkovitch et al. 2021) to gravitational-wave stochastic backgrounds (Abbott et al. 2016a) to, possibly, fast radio bursts (e.g., Zhang 2020). This, then, is an opportune time for a review of the current state of the observations and predictions of compact object merger rates-and, given the expectation of continuing rapid development in this field, it particularly calls for a Living Review.

This review is organised as follows. We provide an executive summary of the observed and theoretically predicted compact-binary merger rates in Sect. 2. We then provide more detailed information and discussion of the observations in Sect. 3 and of the models in Sect. 4 before concluding in Sect. 5. The collated data and code to reproduce all tables and figures in this review are publicly available through Broekgaarden and Mandel (2021), ${ }^{1}$ where we also provide supplementary information on how we obtained the data in the tables and figures.

\section{Executive summary}

We summarise the coalescence rates in tables and figures below: NS-NS binaries in Table 1 and Fig. 1, NS-BH binaries (where we do not distinguish whether the BH or NS formed first) in Table 2 and Fig. 2, and BH-BH binaries in Tables 3 and 4 and Fig. 3.

Coalescence rates are, in general, functions of redshift; we quote current local rates at redshift $z=0$ per unit source time per unit comoving volume in units of $\mathrm{Gpc}^{-3} \mathrm{yr}^{-1}$, but caution that these could be an order of magnitude larger at higher redshifts. Where initially stated in different units, we convert these, using, as appropriate, factors of $1.7 \times 10^{10}$ solar blue-light luminosities per Milky Way equivalent galaxy (MWEG), $1.17 \times 10^{-2}$ MWEG per $\mathrm{Mpc}^{3}$ (Kopparapu et al.

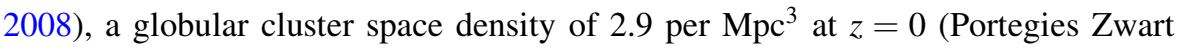

\footnotetext{
${ }^{1}$ The current paper is based on version 5 of the Zenodo data; supplementary material is available on Github.
} 
Table 1 Summary of local NS-NS coalescence rates (GitHub and Zenodo)

\begin{tabular}{|c|c|c|}
\hline Source & Rate $\left[\mathrm{Gpc}^{-3} \mathrm{yr}^{-1}\right]$ & References \\
\hline \multicolumn{3}{|l|}{ Observations: } \\
\hline Gravitational-wave observations, PDB (ind) & $250_{-200}^{+640}$ & GWTC-3; Abbott et al. (2021e) \\
\hline Gravitational-wave observations, MS & $470_{-410}^{+1400}$ & GWTC-3; Abbott et al. (2021e) \\
\hline Gravitational-wave observations, BGP & $99_{-86}^{+260}$ & GWTC-3; Abbott et al. (2021e) \\
\hline Short GRBs & {$[5,1800]$} & Coward et al. (2012) \\
\hline Short GRBs & {$[500,1500]$} & Petrillo et al. (2013) \\
\hline Short GRBs & $270_{-180}^{+1580}$ & Fong et al. (2015) \\
\hline Short GRBs, based on GW170817 & $352_{-281}^{+810}$ & Della Valle et al. (2018) \\
\hline Short GRBs & $1109_{-657}^{+1432}$ & Jin et al. (2018) \\
\hline Short GRBs, based on GW170817 & $190_{-160}^{+440}$ & Zhang et al. (2018) \\
\hline Short GRB, based on GW170817, SWIFT & $160_{-100}^{+200}$ & Dichiara et al. (2020) \\
\hline Kilonovae lower limit & $>8.1$ & Jin et al. (2016) \\
\hline Kilonovae, DES, upper limit & $<24000$ & Doctor et al. (2017) \\
\hline Kilonovae, PTF, upper limit & $<800$ & Kasliwal et al. (2017) \\
\hline Kilonovae, ATLAS, upper limit & $<30000$ & Smartt et al. (2017) \\
\hline Kilonovae, DLT40, upper limit & $<99000$ & Yang et al. (2017) \\
\hline Kilonovae, ZTF, upper limit & $<900$ & Andreoni et al. (2021) \\
\hline Galactic pulsar binaries & $\approx 830_{-680}^{+2110}$ & O’Shaughnessy and Kim (2010) \\
\hline Galactic pulsar binaries & $250_{-160}^{+330}$ & Kim et al. (2015) \\
\hline Galactic pulsar binaries & $450_{-140}^{+290}$ & Pol et al. (2020) \\
\hline Galactic pulsar binaries & $370_{-100}^{+230}$ & Grunthal et al. (2021) \\
\hline \multicolumn{3}{|l|}{ Models: } \\
\hline Isolated binary population synthesis, StarTrack & {$[30,1700]$} & O'Shaughnessy et al. (2010) \\
\hline Isolated binary population synthesis, Scenario Machine & {$[1050,3860]$} & Lipunov and Pruzhinskaya (2014) \\
\hline Isolated binary population synthesis, Brussels code & {$[\leq 1.3,1800]$} & Mennekens and Vanbeveren (2014) \\
\hline Isolated binary population synthesis, StarTrack & {$[30,540]$} & de Mink and Belczynski (2015) \\
\hline Isolated binary population synthesis, StarTrack & {$[52,162]$} & Dominik et al. (2015) \\
\hline Isolated binary population synthesis, BSE & {$[240,1800]$} & Ablimit and Maeda (2018) \\
\hline Isolated binary population synthesis, StarTrack & {$[8,50]$} & Belczynski et al. (2018a) \\
\hline Isolated binary population synthesis, StarTrack & {$[1.5,631]$} & Chruslinska et al. (2018) \\
\hline Isolated binary population synthesis, MOBSE & {$[10,510]$} & Giacobbo and Mapelli (2018) \\
\hline Isolated binary population synthesis, StarTrack & {$[24,68]$} & Klencki et al. (2018) \\
\hline Isolated binary population synthesis, COMBINE & {$[2.7,159]$} & Kruckow et al. (2018) \\
\hline Isolated binary population synthesis, MOBSE & {$[19,591]$} & Mapelli and Giacobbo (2018) \\
\hline Isolated binary population synthesis, COMPAS & {$[61.5,362]$} & Vigna-Gómez et al. (2018) \\
\hline Isolated binary population synthesis, MOBSE & 238 & Artale et al. (2019) \\
\hline Isolated binary population synthesis, MOBSE & {$[12,400]$} & Baibhav et al. (2019) \\
\hline Isolated binary population synthesis, SEVN & 70 & Boco et al. (2019) \\
\hline Isolated binary population synthesis, StarTrack & {$[48,885]$} & Chruslinska et al. (2019) \\
\hline Isolated binary population synthesis, BPASS & {$[339,2178]$} & Eldridge et al. (2019) \\
\hline Isolated binary population synthesis, COMPAS & {$[20,245]$} & Neijssel et al. (2019) \\
\hline
\end{tabular}


Table 1 continued

\begin{tabular}{|c|c|c|}
\hline Source & Rate $\left[\mathrm{Gpc}^{-3} \mathrm{yr}^{-1}\right]$ & References \\
\hline Isolated binary population synthesis, StarTrack & {$[49.3,524]$} & Belczynski et al. (2020) \\
\hline Isolated binary population synthesis, MOBSE & {$[20,640]$} & Giacobbo and Mapelli (2020) \\
\hline Isolated binary population synthesis, MOBSE & $283_{-75}^{+97}$ & Santoliquido et al. (2020) \\
\hline Isolated binary population synthesis, BPASS & {$[394,3190]$} & Tang et al. (2020) \\
\hline Isolated binary population synthesis, COSMIC & {$[600,8900]$} & Zevin et al. (2020) \\
\hline Isolated binary population synthesis, COMPAS & {$[0.32,330]$} & Broekgaarden et al. (2021a, b) \\
\hline Isolated binary population synthesis, BSE, & {$[0.4,1404]$} & Chu et al. (2021) \\
\hline Isolated binary population synthesis, BPASS & {$[43,745]$} & Ghodla et al. (2021) \\
\hline Isolated binary population synthesis, StarTrack & {$[148,322]$} & Olejak et al. (2021) \\
\hline Isolated binary population synthesis, MOBSE & {$[4.3,1036.8]$} & Santoliquido et al. (2021) \\
\hline Hierarchical triples, SecularMultiple & {$[164,3793]$} & Hamers and Thompson (2019) \\
\hline Hierarchical quadruples, MSE & {$[0.8,30.2]$} & Vynatheya and Hamers (2021) \\
\hline Globular cluster dynamics & 30 & Lee et al. (2010) \\
\hline Globular cluster dynamics & {$[0.32,3.2]$} & Bae et al. (2014) \\
\hline Globular cluster dynamics & 121 & Samsing et al. (2014) \\
\hline Globular cluster dynamics, MOCCA & {$[0.02,0.5]$} & Belczynski et al. (2018a) \\
\hline Globular cluster dynamics, CMC & {$[0.009, \lesssim 25.5]$} & Ye et al. (2020) \\
\hline Nuclear star cluster dynamics with SMBH & {$[0.004,1.4]$} & Antonini and Perets (2012) \\
\hline Nuclear star cluster dynamics, with SMBH & $\lesssim 0.02$ & Petrovich and Antonini (2017) \\
\hline Nuclear star cluster dynamics & {$[0.007,0.1]$} & Belczynski et al. (2018a) \\
\hline Nuclear star cluster dynamics, with SMBH & $\lesssim 400$ & McKernan et al. (2020) \\
\hline Nuclear star cluster dynamics, with SMBH & $\gtrsim[0.15,0.3]$ & Wang et al. (2021) \\
\hline Young star clusters & {$[0.03,0.15]$} & Ziosi et al. (2014) \\
\hline Young/Open star clusters, Nbody7 & {$[0.01-0.1]$} & Fragione and Banerjee (2020) \\
\hline Young star clusters, MOBSE & $151_{-38}^{+59}$ & Santoliquido et al. (2020) \\
\hline
\end{tabular}

and McMillan 2000) and a local supernova rate of $1.06 \times 10^{5} \mathrm{Gpc}^{-3} \mathrm{yr}^{-1}$ (Taylor et al. 2014). Of course, such simple re-scalings do not account for the dependence of merger rates on the star formation history and metallicity (de Freitas Pacheco et al. 2006; Belczynski et al. 2010; Dvorkin et al. 2016; Neijssel et al. 2019; Chruslinska and Nelemans 2019; Mapelli 2021).

In general, we follow original papers in stating the rates; a discussion follows in the next two sections. Where uncertainties are stated, we try to indicate them with \pm subscripts and superscripts; however, these are not always available, and may not be attributable to a specific confidence interval when available. Sometimes, we state a range in square brackets without a central value.

The vast literature on compact-object merger rate predictions dating back to at least 1979 (Clark et al. 1979) makes a complete historical review implausible. Therefore, we focus on the latest contributions from each group, except where contributions use significantly different methodology or examine the impact of 


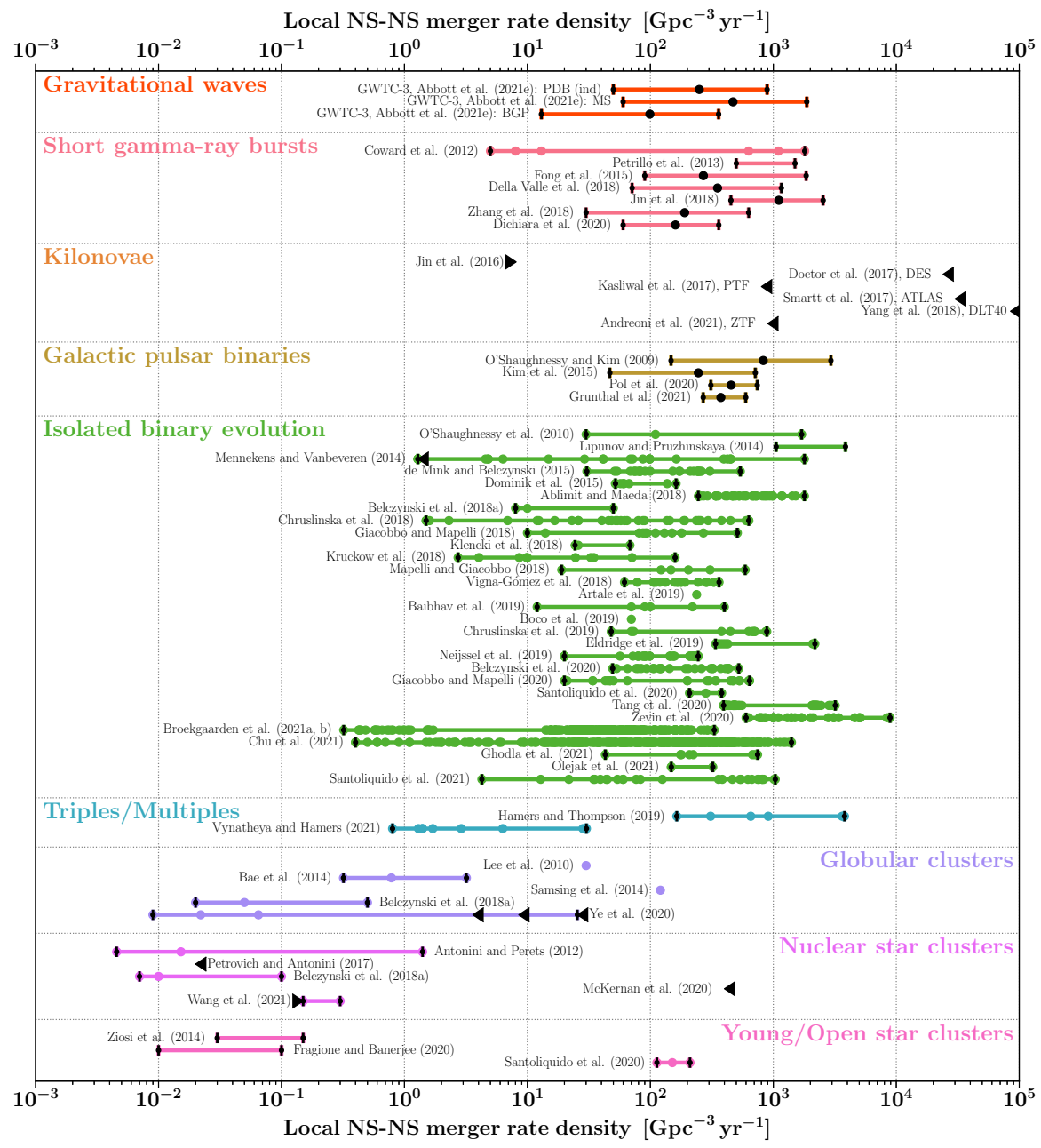

Fig. 1 NS-NS merger rates from Table 1. Black lines denote the interval boundaries. Triangles mark upper and lower limits. Colored circles mark inferred or simulated values, where multiple values are given in a study. Black circles mark the median or mean of a confidence interval, where one is provided. (Standalone PDF image in this article's Supplementary Material. Source code: GitHub)

different assumptions. We generally eschew papers published before 2010 as these have typically been superseded by observational and theoretical advances.

We combine rates into several broad categories for ease of viewing, but multiple different channels with a broad range of physics may be at play within any one category. For example, the category of "nuclear star clusters" may include dynamical captures in nuclear clusters without a massive black hole, mergers in hierarchical triples involving a massive black hole, or mergers aided by an accretion disk in an active galactic nucleus. We do not include some very rare channels with predicted rates below the range shown in the figures. 
Table 2 Summary of local NS-BH coalescence rates (GitHub and Zenodo)

Source Rate $\left[\mathrm{Gpc}^{-3} \mathrm{yr}^{-1}\right]$ References

Observations:

Gravitational-wave observations, PDB (ind)

Gravitational-wave observations, MS

Gravitational-wave observations, BGP

Galactic pulsar binaries

Models:

Isolated binary population synthesis, StarTrack Isolated binary population synthesis, Brussels code Isolated binary population synthesis, StarTrack Isolated binary population synthesis, StarTrack Isolated binary population synthesis, BSE Isolated binary population synthesis, MOBSE Isolated binary population synthesis, StarTrack Isolated binary population synthesis, COMBINE Isolated binary population synthesis, MOBSE Isolated binary population synthesis, MOBSE Isolated binary population synthesis, MOBSE Isolated binary population synthesis, SEVN Isolated binary population synthesis, StarTrack Isolated binary population synthesis, BPASS Isolated binary population synthesis, COMPAS Isolated binary population synthesis, StarTrack Isolated binary population synthesis, MOBSE Isolated binary population synthesis, MOBSE Isolated binary population synthesis, BPASS Isolated binary population synthesis, COSMIC Isolated binary population synthesis, COMPAS Isolated binary population synthesis, BPASS Isolated binary population synthesis, StarTrack Isolated binary population synthesis, POSYDON Isolated binary population synthesis, MOBSE Isolated binary population synthesis, BSE

Chemically homogeneous evolution, MESA

Population III stars

Hierarchical triples

Hierarchical triples, SecularMultiple

Hierarchical triples in young star clusters, OKINAMI, Hierarchical quadruples, MSE

Globular cluster dynamics

Globular cluster dynamics, ARCHAIN

Globular cluster dynamics, CMC

$170_{-89}^{+150}$
$57_{-42}^{+120}$
$32_{-25}^{+62}$
$\lesssim 1800$

GWTC-3; Abbott et al. (2021e)

GWTC-3; Abbott et al. (2021e)

GWTC-3; Abbott et al. (2021e)

Pol et al. (2021)

$[10,280]$

O'Shaughnessy et al. (2010)

$[0.06,800]$

$[9,115]$

$[0.04,20]$

[1, 160]

[5, 780]

[13.3, 26.7]

$[2,53]$

[9, 115]

78

[4, 37]

20

$[5,230]$

$[209,269]$

[19, 204]

[0.48, 297]

$[6,80]$

$49_{-34}^{+48}$

[58, 6225]

[3.7, 1100]

$[2.2,830]$

[8.7, 498]

$[4,16]$

$[5.7,77]$

$[1.8,128]$

$[10,72]$

$[0.02,0.2]$

[0.0002, 0.016]

$\left[1.9 \times 10^{-4}, 22\right]$

$[345,680]$

$[0.04,0.34]$

$[5.7,57.1]$

[0.01, 0.12]

$\lesssim 0.1$

$[0.009, \lesssim 5.5]$
Mennekens and Vanbeveren (2014)

de Mink and Belczynski (2015)

Dominik et al. (2015)

Ablimit and Maeda (2018)

Giacobbo and Mapelli (2018)

Klencki et al. (2018)

Kruckow et al. (2018)

Mapelli and Giacobbo (2018)

Artale et al. (2019)

Baibhav et al. (2019)

Boco et al. (2019)

Chruslinska et al. (2019)

Eldridge et al. (2019)

Neijssel et al. (2019)

Belczynski et al. (2020)

Giacobbo and Mapelli (2020)

Santoliquido et al. (2020)

Tang et al. (2020)

Zevin et al. (2020)

Broekgaarden et al. (2021a, b)

Ghodla et al. (2021)

Olejak et al. (2021)

Román-Garza et al. (2021)

Santoliquido et al. (2021)

Shao and Li (2021)

Marchant et al. (2017)

Belczynski et al. (2017)

Fragione and Loeb (2019a, 2019b)

Hamers and Thompson (2019)

Trani et al. (2021)

Vynatheya and Hamers (2021)

Clausen et al. (2013)

Arca Sedda (2020b)

Ye et al. (2020) 
Table 2 continued

\begin{tabular}{lll}
\hline Source & Rate $\left[\mathrm{Gpc}^{-3} \mathrm{yr}^{-1}\right]$ & References \\
\hline Nuclear star cluster dynamics, with SMBH & {$[0.02,0.4]$} & Petrovich and Antonini (2017) \\
Nuclear star cluster dynamics, with SMBH & $\gtrsim[2,5]$ & Stephan et al. (2019) \\
Nuclear star cluster dynamics, & $\lesssim 0.01$ & Arca Sedda (2020b) \\
Nuclear star cluster dynamics, with SMBH & $\lesssim 300$ & McKernan et al. (2020) \\
Nuclear star cluster dynamics, with SMBH & $\gtrsim[0.15,0.3]$ & Wang et al. (2021) \\
Young star clusters, starlab & $\lesssim 0.1$ & Ziosi et al. (2014) \\
Young/Open star clusters, Nbody7 & $\lesssim 3 \times 10^{-3}$ & Fragione and Banerjee (2020) \\
Young star clusters, MOBSE & $\lesssim 28$ & Rastello et al. (2020) \\
Young star clusters, MOBSE & $41_{-23}^{+33}$ & Santoliquido et al. (2020) \\
Young star clusters, & {$[0.04,36.6]$} & Arca Sedda (2021) \\
\hline
\end{tabular}

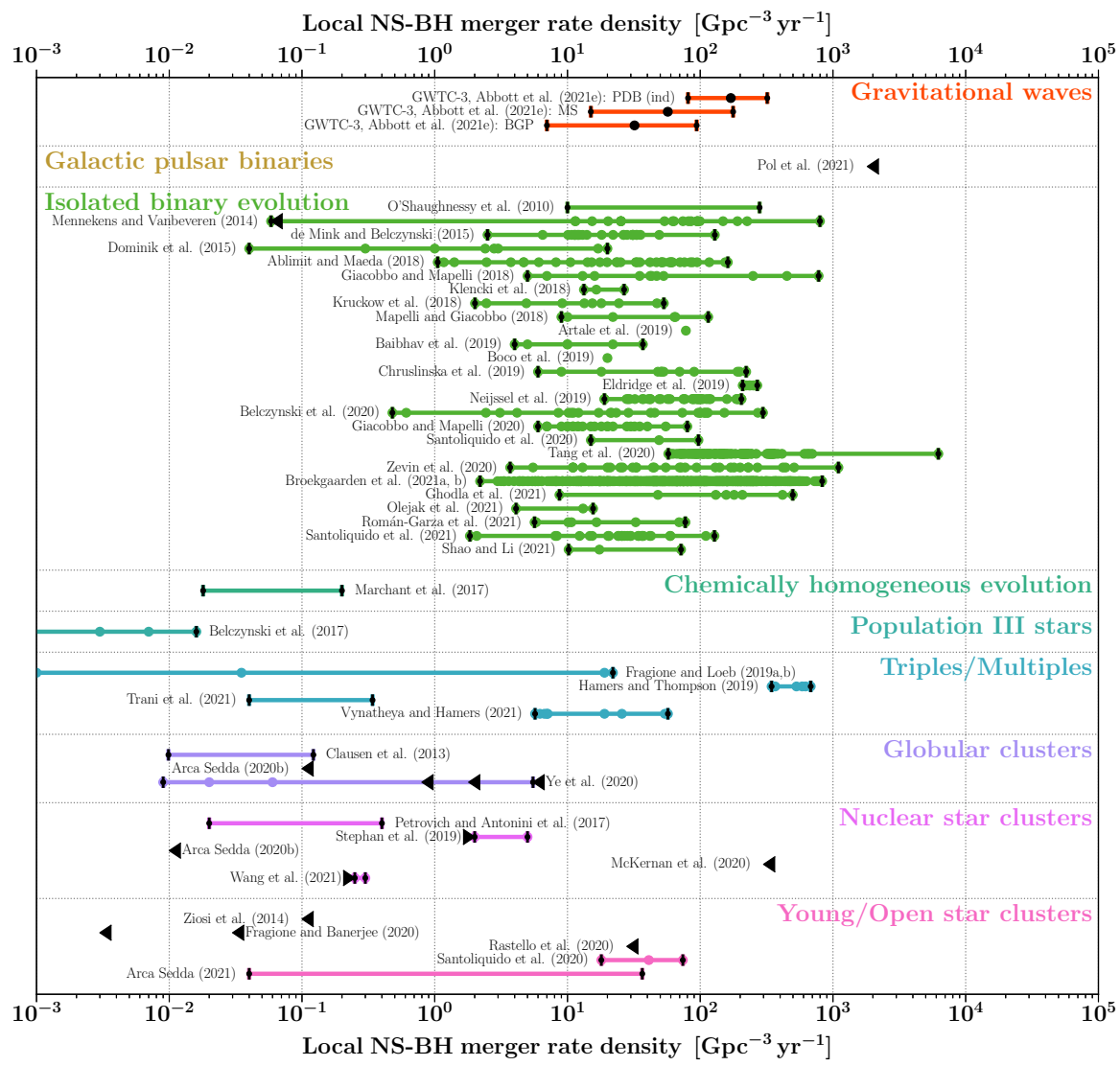

Fig. 2 NS-BH merger rates from Table 2. Notation as in Fig. 1. (Standalone PDF image in this article's Supplementary Material. Source code: GitHub) 
Table 3 Summary of predictions of local BH-BH coalescence rates, part 1: observations and isolated binary evolution models (GitHub and Zenodo)

\begin{tabular}{lll}
\hline Source & Rate & References \\
& {$\left[\mathrm{Gpc}^{-3} \mathrm{yr}^{-1}\right]$} & \\
\hline
\end{tabular}

Observations:

Gravitational-wave observations, PDB (ind)

Gravitational-wave observations, MS

Gravitational-wave observations, BGP

Gravitational-wave observations, $z$-dependent

Models:

Isolated binary population synthesis, StarTrack

Isolated binary population synthesis, Brussels code

Isolated binary population synthesis, StarTrack

Isolated binary population synthesis, StarTrack

Isolated binary population synthesis, BSE

Isolated binary population synthesis, Scenario Machine

Isolated binary population synthesis, MOBSE

Isolated binary population synthesis, BSE

Isolated binary population synthesis, StarTrack

Isolated binary population synthesis, MOBSE

Isolated binary population synthesis, StarTrack

Isolated binary population synthesis, COMBINE

Isolated binary population synthesis, MOBSE

Isolated binary population synthesis, MOBSE

Isolated binary population synthesis, MOBSE

Isolated binary population synthesis, StarTrack

Isolated binary population synthesis, BPASS

Isolated binary population synthesis, COMPAS

Isolated binary population synthesis, SEVN

Isolated binary population synthesis, StarTrack

Isolated binary population synthesis, MOBSE

Isolated binary population synthesis, MOBSE

Isolated binary population synthesis, BPASS

Isolated binary population synthesis, COSMIC

Isolated binary population synthesis, POSYDON

Isolated binary population synthesis, COMPAS,

Isolated binary population synthesis, BPASS

Isolated binary population synthesis, MOBSE

Isolated binary population synthesis, StarTrack

Isolated binary population synthesis, COMPAS

Isolated binary population synthesis, POSYDON

Isolated binary population synthesis, MOBSE

Isolated binary population synthesis, BSE

Chemically homogeneous evolution

Chemically homogeneous evolution, MESA

$\begin{array}{ll}22_{-6}^{+9} & \text { GWTC-3; Abbott et al. (2021e) } \\ 42_{-20}^{+88} & \text { GWTC-3; Abbott et al. (2021e) } \\ 33_{-10}^{+16} & \text { GWTC-3; Abbott et al. (2021e) } \\ 18_{-8}^{+12} & \text { GWTC-3; Abbott et al. (2021e) }\end{array}$

Rate

$[2,40]$
$[\leq 96,1140]$
$[14,2500]$
$[0.5,221]$
850
100

$[20,572]$

[20, 320]

$[32,1072]$

$[43,1500]$

$[89,203]$

$[0.6,109]$

$[146,240]$

142

$[30,60]$

$[12,1072]$

[56, 174].

[59, 1157]

90

[1.24, 1368]

[43, 160]

$50_{-37}^{+71}$

[10, 219]

[84, 6900]

[39, 170]

[3.8, 810]

[31, 873]

[6, 37]

$[18,89]$

[51, 87]

[70, 203]

[10, 105.4]

$[43,76]$

$[2,80]$

$[0.7,16]$
O'Shaughnessy et al. (2010)

Mennekens and Vanbeveren (2014)

de Mink and Belczynski (2015)

Dominik et al. (2015)

Lamberts et al. (2016)

Lipunov et al. (2017)

Mapelli et al. (2017)

Ablimit and Maeda (2018)

Chruslinska et al. (2018)

Giacobbo and Mapelli (2018)

Klencki et al. (2018)

Kruckow et al. (2018)

Mapelli and Giacobbo (2018)

Artale et al. (2019)

Baibhav et al. (2019)

Chruslinska et al. (2019)

Eldridge et al. (2019)

Neijssel et al. (2019)

Spera et al. (2019)

Belczynski et al. (2020)

Giacobbo and Mapelli (2020)

Santoliquido et al. (2020)

Tang et al. (2020)

Zevin et al. (2020)

Bavera et al. (2021)

Broekgaarden et al. (2021a, b)

Ghodla et al. (2021)

Mapelli et al. (2021)

Olejak et al. (2021)

Riley et al. (2021)

Román-Garza et al. (2021)

Santoliquido et al. (2021)

Shao and Li (2021)

Mandel and de Mink (2016)

Marchant et al. (2016) 
Table 3 continued

\begin{tabular}{lll}
\hline Source & $\begin{array}{l}\text { Rate } \\
{\left[\mathrm{Gpc}^{-3} \mathrm{yr}^{-1}\right]}\end{array}$ & References \\
\hline $\begin{array}{l}\text { Chemically homogeneous evolution, MESA } \\
\text { Chemically homogeneous evolution, COMPAS }\end{array}$ & {$[5.8,7]$} & $\begin{array}{l}\text { du Buisson et al. (2020) } \\
\text { Riley et al. (2021) }\end{array}$ \\
$\begin{array}{l}\text { Population III stars } \\
\text { Population III stars, StarTrack }\end{array}$ & {$[12,25]$} & Kinugawa et al. (2014) \\
Population III stars, BSE & {$[0.016,1.9]$} & Belczynski et al. (2017) \\
Population III stars, BSE & {$[0.38,2.9]$} & Hijikawa et al. (2021) \\
Population III stars in Nuclear Star Clusters & {$[0.13,0.66]$} & Kinugawa et al. (2021) \\
Population III stars, BSE & {$[0.02,0.6]$} & Liu and Bromm (2021) \\
\end{tabular}

Table 4 Summary of predictions of local BH-BH coalescence rates, part 2: dynamical formation models and primordial black holes (GitHub and Zenodo)

\begin{tabular}{|c|c|c|}
\hline Source & $\begin{array}{l}\text { Rate } \\
{\left[\mathrm{Gpc}^{-3} \mathrm{yr}^{-1}\right]}\end{array}$ & References \\
\hline Hierarchical triples, Rebound package & {$[0.14,6.3]$} & Silsbee and Tremaine (2017) \\
\hline Hierarchical triples, TRES & {$[0.3,2.5]$} & Antonini et al. (2017) \\
\hline Hierarchical triples & {$[2,25]$} & Rodriguez and Antonini (2018) \\
\hline Hierarchical triples in globular clusters, CMC & {$[\gtrsim 0.35,1]$} & Martinez et al. (2020) \\
\hline Hierarchical triples in young star clusters, OKINAMI, & {$[0.2,1.44]$} & Trani et al. (2021) \\
\hline Hierarchical quadruples, MSE & {$[8.5,39.8]$} & Vynatheya and Hamers (2021) \\
\hline Globular cluster dynamics & {$[7.25,29]$} & Bae et al. (2014) \\
\hline Globular cluster dynamics, CMC & {$[3.8,13]$} & Rodriguez et al. (2015) \\
\hline Globular cluster dynamics & 5 & Antonini and Rasio (2016) \\
\hline Globular cluster dynamics, CMC & {$[2,20]$} & Rodriguez et al. (2016a) \\
\hline Globular cluster dynamics, MOCCA & {$[5.4,30]$} & Askar et al. (2017) \\
\hline Globular cluster dynamics & {$[13,57]$} & Fujii et al. (2017) \\
\hline Globular cluster dynamics, Nbody6 & {$[6.5,26]$} & Park et al. (2017) \\
\hline Globular cluster dynamics & {$[4,60]$} & Fragione and Kocsis (2018) \\
\hline Globular cluster dynamics & {$[0.8,20]$} & Hong et al. (2018) \\
\hline Globular cluster dynamics, CMC & {$[4,18]$} & Rodriguez and Loeb (2018) \\
\hline Globular cluster dynamics & $\approx 6$ & Choksi et al. (2019) \\
\hline Globular cluster dynamics, cBHBd & {$[0.2,50]$} & Antonini and Gieles (2020) \\
\hline Globular cluster dynamics, CMC & {$[9,30]$} & Kremer et al. (2020) \\
\hline Globular cluster dynamics, MOBSE & {$[0.8,7]$} & Mapelli et al. (2021) \\
\hline Nuclear star cluster dynamics, without SMBH & {$[1,10]$} & Miller and Lauburg (2009) \\
\hline Nuclear star cluster dynamics, with SMBH & {$[0.002,0.6]$} & Antonini and Perets (2012) \\
\hline Nuclear star cluster dynamics, without SMBH & 1.5 & Antonini and Rasio (2016) \\
\hline Nuclear star cluster dynamics:, with SMBH & $\sim 1.2$ & Bartos et al. (2017) \\
\hline Nuclear star cluster dynamics, with SMBH & {$[0.6,15]$} & Petrovich and Antonini (2017) \\
\hline Nuclear star cluster dynamics, with SMBH & $\sim 3$ & Stone et al. (2017) \\
\hline Nuclear star cluster dynamics, with SMBH & {$[0.01,0.4]$} & Hamers et al. (2018) \\
\hline Nuclear star cluster dynamics, with SMBH & {$[1,3]$} & Hoang et al. (2018) \\
\hline
\end{tabular}


Table 4 continued

\begin{tabular}{|c|c|c|}
\hline Source & $\begin{array}{l}\text { Rate } \\
{\left[\mathrm{Gpc}^{-3} \mathrm{yr}^{-1}\right]}\end{array}$ & References \\
\hline Nuclear star cluster dynamics, with SMBH & {$[0.002,0.04]$} & Rasskazov and Kocsis (2019) \\
\hline Nuclear star cluster dynamics, with SMBH & $\gtrsim[7,15]$ & Stephan et al. (2019) \\
\hline Nuclear star cluster dynamics with SMBH & {$[3.3,8.6]$} & Arca Sedda (2020a) \\
\hline Nuclear star cluster dynamics with SMBH & {$[0.002,18]$} & Gröbner et al. (2020) \\
\hline Nuclear star cluster dynamics & {$[0.09,10]$} & Mapelli et al. (2021) \\
\hline Nuclear star cluster dynamics, with SMBH & {$[0.02,60]$} & Tagawa et al. (2020) \\
\hline Nuclear star cluster dynamics, with SMBH & {$[0.1,1.6]$} & Yang et al. (2020) \\
\hline Nuclear star cluster dynamics, with SMBH & {$[6,20]$} & Ford and McKernan (2021) \\
\hline Nuclear star cluster dynamics, with SMBH & $\geq[0.3,5]$ & Wang et al. (2021) \\
\hline Young star clusters, starlab & $\lesssim 1.5$ & Ziosi et al. (2014) \\
\hline Young star clusters, starlab & $\gtrsim 1$ & Mapelli (2016) \\
\hline Open star clusters, NBODY7 & $\lesssim 2$ & Rastello et al. (2019) \\
\hline Young star clusters, NBODY6 & $\lesssim[55,110]$ & Di Carlo et al. (2020) \\
\hline Open star clusters, NBODY6 & {$[35,70]$} & Kumamoto et al. (2020) \\
\hline Young star clusters, MOBSE & $64_{-20}^{+34}$ & Santoliquido et al. (2020) \\
\hline Young and open star clusters, NBODY7 & {$[0.5,37.9]$} & Banerjee (2021) \\
\hline Young star clusters, MOBSE & {$[0.1,18]$} & Mapelli et al. (2021) \\
\hline Young star clusters, NBODY6 MOBSE & $88_{-26}^{+34}$ & Rastello et al. (2021) \\
\hline Primordial binaries & {$[0.02, \lesssim 3]$} & Bird et al. (2016) \\
\hline Primordial binaries & $\lesssim\left[0.2,10^{5}\right]$ & Ali-Haïmoud et al. (2017) \\
\hline Primordial binaries & $\lesssim\left[6,10^{4}\right]$ & Raidal et al. (2019) \\
\hline
\end{tabular}

We do not quote predictions for the number of detections per year for a given instrument because such predictions must take into account the variation in both the merger rate and the mass distribution of merging binaries with redshift for detectors sensitive to cosmological distances, and this information is frequently not readily available in the literature. Derivations of how to do such calculations are provided by, e.g., Belczynski et al. (2014) and de Mink and Mandel (2016); both analytical fits (e.g., Fishbach and Holz 2017) and numerical codes (e.g., Gerosa 2017; Team COMPAS: Riley et al. 2021) are available.

\section{Observed rates}

In this section, we discuss the latest observational constraints on compact-object coalescence rates. There are direct observations of compact binary mergers, either through gravitational waves or through electromagnetic transients accompanying such mergers, particularly short gamma-ray bursts. Alternatively, there are observations of binaries that are not yet merging, but are expected to do so as compact objects in the future, such as Galactic double neutron stars and perhaps some high-mass X-ray binaries. 


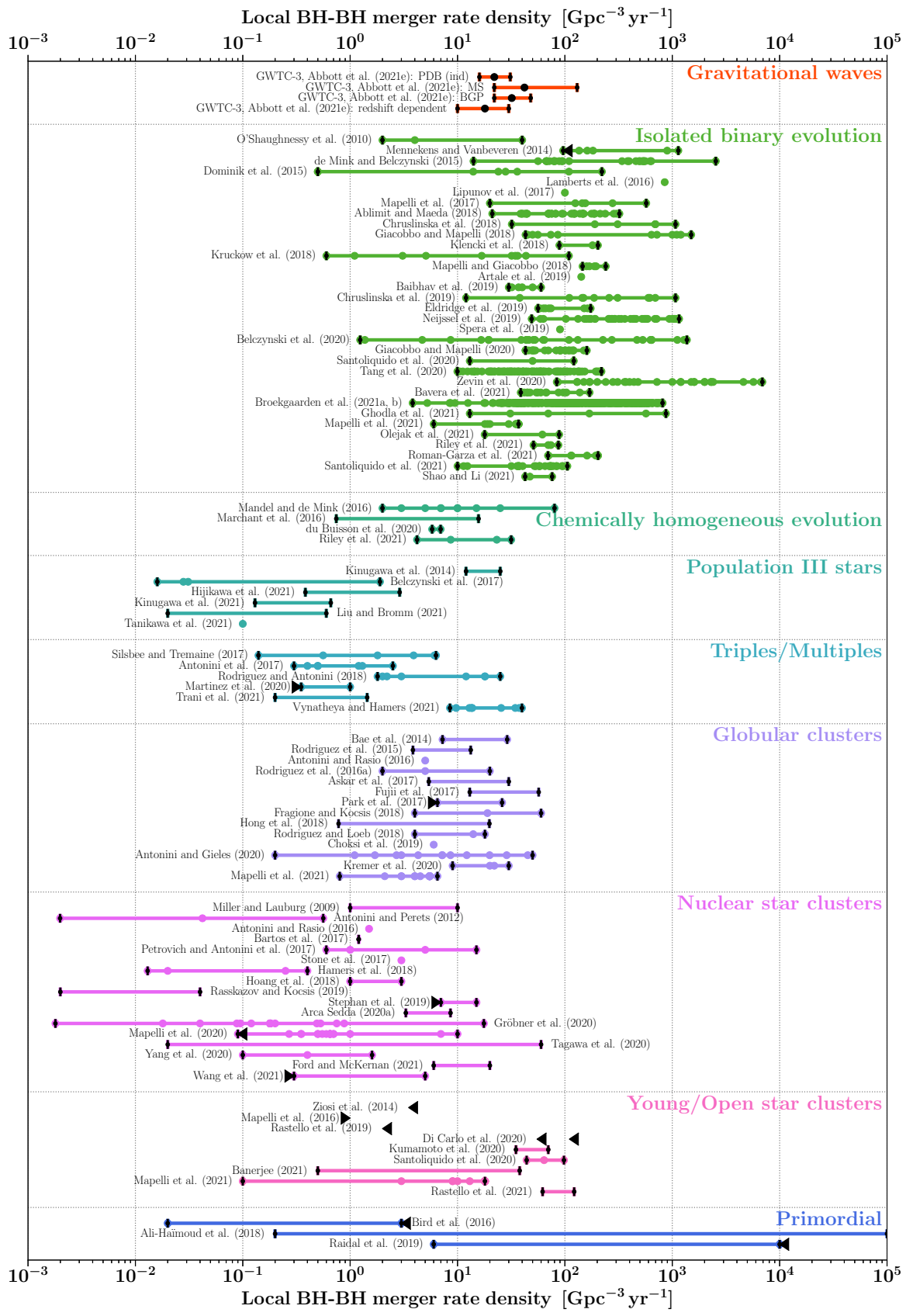

Fig. 3 BH-BH merger rates from Table 3. Notation as in Fig. 1. (Standalone PDF image in this article's Supplementary Material. Source code: GitHub) 
In all cases the key challenge in accurately inferring rates from limited observations comes from significant selection effects, which may be particularly difficult to quantify when the data set is inhomogeneous, and/or when the distribution of the intrinsic loudness of the signals is not known. Moreover, in some cases there can be uncertainty in the interpretation of the signals. Meanwhile, for systems that are not yet merging, there may be uncertainties in their future evolution. We discuss these challenges below.

\subsection{Gravitational waves}

Gravitational-wave data should, at first glance, enable straightforward rate estimates. These are direct observations of compact-object coalescences. The data are homogeneous, with well-understood selection effects which can be modelled by injecting mock signals into the data stream and measuring how many of them will be successfully extracted by the search pipelines. Once the surveyed time $\times$ volume of the instruments is thus estimated, the volumetric merger rate is given by the number of detections in this time $\times$ volume. We might therefore expect that analysing some 70 confident $\mathrm{BH}-\mathrm{BH}$ mergers from the first, second, and third advanced detector observing run in Abbott et al. (2021e) would enable the BH-BH merger rate to be inferred to a fractional accuracy of $1 / \sqrt{70} \approx 12 \%$ based on Poisson statistics.

However, there are several complicating factors to this story, as a result of which the total binary black hole merger rate is still uncertain at the factor of two level. Firstly, the detection rate is a sensitive function of mass, with more massive binaries typically providing higher-amplitude gravitational-wave signals that can be detected through a greater volume. Therefore, inferring the total merger rate relies on simultaneously measuring the mass distribution of merging black holes, which requires many more observations (Abbott et al. 2021e). One way to partially overcome this uncertainty is to focus on observations in a more narrow mass band (Roulet et al. 2020; Abbott et al. 2021c); for example, Roulet et al. (2020) find that the merger rate of $20-30 M_{\odot} \mathrm{BHs}^{2}$ is $1.5-5.3 \mathrm{Gpc}^{-3} \mathrm{yr}^{-1}$, while Abbott et al. (2021c) find a $\mathrm{BH}-\mathrm{BH}$ merger rate in the same mass range of $3.7_{-1.1}^{+1.6}$ or $2.6_{-1.3}^{+1.9}$ $\mathrm{Gpc}^{-3} \mathrm{yr}^{-1}$ depending on the assumed mass distributions. The inferred merger rates reported in the summary tables and figures as "PDB (ind)", "MS", and "BGP" refer to the corresponding mass distribution models described in Abbott et al. (2021e): Power law + dip + break (with independent mass pairings for the two components), Power law + peak multi-source (separate BH mass models in $\mathrm{BH}-\mathrm{BH}$ and NS-BH binaries) and Binned Gaussian process (unmodelled with regularisation).

Secondly, another uncertainty arises from the possibility that the merger rate varies with redshift, for which there is already significant support in the data (Fishbach et al. 2021; Abbott et al. 2021e). Allowing for the variation of the merger rate with redshift (but not yet of the mass distribution with redshift, although some

\footnotetext{
$\overline{{ }^{2} \text { We generally use solar masses }}\left(M_{\odot}\right)$ and solar radii $\left(R_{\odot}\right)$ as mass and distance units.
} 
theoretical models predict this) further shifts the binary black hole merger rate at redshift $z=0$. The " $z$-dependent" rate reported for BH-BH mergers comes from the redshift-dependent merger rate model evaluated at $z=0$ (Abbott et al. 2021e).

Thirdly, details of search methodology and tuning can impact the set of detections (e.g., Abbott et al. 2021b, d), and independent searches over the publicly released LIGO-Virgo data have yielded slightly different source populations (e.g., Zackay et al. 2019; Venumadhav et al. 2020; Nitz et al. 2021a, b). While searches with slightly different sensitivities and different numbers of detections should still yield consistent rate estimates, some fluctuation is to be expected, especially through the impact of different inferred mass distributions as described above.

Fourthly, while the chirp mass $m_{1}^{3 / 5} m_{2}^{3 / 5}\left(m_{1}+m_{2}\right)^{-1 / 5}$ is measured accurately for low-mass binaries through gravitational-wave signals, the mass ratio measurement is much less accurate, making it difficult to distinguish neutron stars from low-mass black holes (see, e.g., Hannam et al. 2013). A measurement of tidal deformation could point unambiguously to a neutron star rather than a black hole, but such measurements require sensitivity at higher frequencies, and only upper limits have been placed so far (Abbott et al. 2017b). Therefore, in the absence of an electromagnetic counterpart, it is not clear how to classify a system such as GW190814 (Abbott et al. 2020d), which consisted of a $\approx 23 M_{\odot}$ BH coalescing with $\mathrm{a} \approx 2.6 M_{\odot}$ compact object that is probably a low-mass black hole but could be a very high-mass neutron star.

Estimates of the NS-NS and NS-BH merger rates are based on a very small number of confident detections, including GW170817 and GW190425 in the former category and GW200105 and GW200115 in the latter category (Abbott et al. 2017b, 2020a, 2021a). Furthermore, these estimates are particularly sensitive to assumptions about the poorly constrained mass distributions in NS-NS and NS-BH binaries.

\subsection{Short gamma-ray bursts and kilonovae}

Mergers of two neutron stars have long been expected to be accompanied by a short burst of spectrally hard gamma radiation accompanying an ultra-relativistic jet, with subsequent synchrotron radiation afterglow from the interaction of the jet with the circumstellar medium (Paczyński and Rhoads 1993; Rosswog and Ramirez-Ruiz 2002; Nakar 2007). Meanwhile, the formation and subsequent radioactive decay of neutron-rich elements through r-process nucleosynthesis was conjectured to power an optical kilonova (Li and Paczyński 1998; Rosswog et al. 1999; Metzger et al. 2010; Kasen et al. 2013). Both expectations were spectacularly confirmed with the detection of a short gamma-ray burst (Abbott et al. 2017a), followed by the observation of a kilonova (Abbott et al. 2017c; Kasen et al. 2017; Metzger 2019), and finally a broad-spectrum (radio through optical to X-ray) afterglow (Mooley et al. 2018; Lyman et al. 2018; Troja et al. 2017) following the NS-NS merger GW17017.

One concern with using short gamma-ray bursts (SGRBs) to infer binary neutron star coalescence rates is the uncertainty in a one-to-one association between these 
events. On the one hand, some SGRBs and perhaps even kilonovae could be associated with NS-BH mergers (Troja et al. 2008; Berger 2014; Li et al. 2017; Gompertz et al. 2020). However, this is likely only possible for those events when the neutron star is tidally disrupted at a sufficient distance from the black hole, requiring the black hole to be low in mass and/or rapidly rotating (Foucart et al. 2018). Therefore, the contribution of NS-BH mergers to SGRBs is likely low (e.g., Drozda et al. 2020). On the other hand, given the challenges in reproducing ultrarelativistic jet formation in numerical merger simulations (Mösta et al. 2020), it cannot be determined with confidence whether all neutron star mergers should be accompanied by SGRBs.

Nevertheless, SGRBs have been used for more than a decade as proxies for the NS-NS merger rate, with Nakar et al. (2006) estimating the merger rate as at least $10 \mathrm{Gpc}^{-3} \mathrm{yr}^{-1}$, but possibly several orders of magnitude higher. The key challenge is the uncertainty in the angular size of the jet: the smaller the jet, the smaller fraction of SGRBs beamed toward Earth, and hence the larger the intrinsic rate based on the observed rate of SGRBs. Fong et al. (2012) used evidence for jet breaks to constrain the angular size of the jet, allowing them to infer an NS-NS merger rate. However, the evidence from GW170817 firmly indicated that the jet has spatial structure, rather than a simple top-hat profile (Troja et al. 2018; Resmi et al. 2018; Lamb et al. 2019). This requires a re-evaluation of earlier models, an ongoing effort (Paul 2018); for example, Margutti and Chornock (2021, see their Fig. 3) estimate the rate of GW170817A-like SGRBs as $\gtrsim 300 \mathrm{Gpc}^{-3} \mathrm{yr}^{-1}$ given the observed off-axis angle under the assumption that other SGRBs would have similar jet profiles.

The first kilonova observation was made in 2013 (Tanvir et al. 2013). While more candidates have been observed in the last few years, they are still difficult to unambiguously distinguish from other transients. At present, their luminosity function is not yet sufficiently well known to infer an accurate rate. However, the absence of transients with a kilonova signal similar to that accompanying GW170187 in data from the Palomar Transient Factor allowed Kasliwal et al. (2017) to place a conservative 3- $\sigma$ upper limit of $800 \mathrm{Gpc}^{-3} \mathrm{yr}^{-1}$. Andreoni et al. (2021, see their Fig. 9) compile a list of optical kilonova surveys with upper limits between 900 and $99000 \mathrm{Gpc}^{-3} \mathrm{yr}^{-1}$.

\subsection{Pulsars in Galactic compact object binaries}

Observations of double compact object binaries prior to merger are the next best thing to direct merger observations for measuring merger rates. Provided that the masses, period or separation, and eccentricity of the binary are known, the time to merger through the emission of gravitational waves can be computed (Peters 1964). Assuming a steady-state configuration, which is a reasonable assumption for our Galaxy with its slowly evolving metallicity and star formation rate, an observed population of pre-merger compact object binaries yields an estimate of the coalescence rate. 
So far, NS-NS binaries are the only double compact objects for which direct observations are available. Nearly 20 such binaries have been observed in the Galaxy through radio pulsar pulsar observations (see, e.g., Tauris et al. 2017; Farrow et al. 2019, for recent compilations). Of course, these are expected to represent a relatively small fraction of all Galactic double neutron stars, with most remaining unobserved because neither neutron star is a pulsar or any pulsars are too dim or beamed away from the Earth. Moreover, pulsars in the tightest binaries are rare because of their rapid inspiral and may be challenging to detect because the pulsations are Doppler shifted by orbital modulation. Therefore, the key challenge in inferring the merger rate of close double neutron stars in the Galaxy from these observations lies in accounting for selection effects, particularly the uncertain luminosity function and beaming fraction of pulsars.

Fortunately, the rapidly growing amount of pulsar data since the pioneering efforts of Phinney (1991) and Narayan et al. (1991) to infer NS-NS merger rates from binary pulsar observations enables increasingly accurate treatments of these selection effects. Much of the recent literature follows the statistical framework of Kim et al. (2003), with the addition of varying models of the luminosity distribution (Kalogera et al. 2004), more sophisticated models of the pulsar beaming fraction which could vary with pulsar age (O'Shaughnessy and Kim 2010), an attempt to account for the observation of both pulsars in the double pulsar J0737-3039 (Kim et al. 2015) and the addition of new systems (with the relatively small number of known Galactic binaries, a single new short-lived double neutron star could impact the rate estimate at the factor of two level, as shown by Kim et al. 2006). We report the rates from a few recent papers to indicate the evolving estimates of the double neutron star merger rate (see Abadie 2010, for a compilation including older results).

There are several complicating factors in extracting coalescence rates from the observed Galactic double neutron stars. B2127+11C and possibly J1807-2500 are known double neutron stars in Galactic globular clusters; these are generally excluded from rate estimates because they are believed to be rare dynamically formed systems (Ye et al. 2020, but see Andrews and Mandel 2019). Another concern is that the detectability of a Galactic double neutron star may correlate with the evolutionary channel and with the system properties, e.g., through the amount of recycling the pulsar experiences. This could lead some sub-populations of pulsarsperhaps the more massive ones like GW190425 (Abbott et al. 2020a; Romero-Shaw et al. 2020; Vigna-Gómez et al. 2021a) - to be missed in Galactic surveys. Moreover, extrapolation from the Milky Way merging double neutron star rate to a volumetric rate depends on the accuracy of estimating the local star formation rate (de Freitas Pacheco et al. 2006) and on the sensitivity of the yield of merging double neutron stars to metallicity.

No Galactic radio pulsars in NS-BH binaries have been detected so far. However, the lack of such observations only weakly constrains the NS-BH merger rate from above. If the heavier $\mathrm{BH}$ forms first, which appears intuitive given the shorter lifetimes of more massive stars, the NS will not be recycled by accretion, which limits the time during which it can be observed as a radio pulsar (Chattopadhyay et al. 2021). Mass transfer can invert the mass ratio, allowing the 
NS to form first from the originally more massive star; however, the formation rate of such systems is likely quite low (Pfahl et al. 2005).

\subsection{High-mass X-ray binaries}

As we will discuss in the next section, most compact object binaries formed through isolated binary evolution are expected to go through a high-mass X-ray binary stage, in which the first companion to form a black hole or neutron star accretes from the wind of the stellar companion. This phase may directly precede the formation of two compact objects, and can therefore be used to constrain compact binary coalescence rates. For example, Bulik et al. (2011) used the extragalactic high-mass BH X-ray binaries IC10 X-1 and NGC300 X-1 to estimate the merger rate of $\mathrm{BH}-\mathrm{BH}$ binaries at $360_{-260}^{+500} \mathrm{Gpc}^{-3} \mathrm{yr}^{-1}$.

However, such estimates suffer from significant uncertainties. Firstly, there are often large systematic errors in the masses and separations of the observed systems; in particular, the masses of IC10 X-1 and NGC300 X-1 may be much lower than in the analysis of Bulik et al. (2011) because orbital velocities are very challenging to measure in the presence of optically thick Wolf-Rayet winds (Laycock et al. 2015). Even well-studied high-mass X-ray binaries such as Cygnus X-1 (Orosz et al. 2011) may require significant corrections to their masses (Miller-Jones et al. 2021), while the uncertain present properties of Cygnus X-3 translate into uncertainty about its future fate (Belczynski et al. 2013). Secondly, the observed systems typically come from a heterogeneous set of observations, complicating the determination of selection effects. Thirdly, the future evolution of such systems is sensitive to assumptions about the dynamical stability of any future mass transfer episodes (van den Heuvel et al. 2017), the amount of mass loss in winds (cf. Belczynski et al. 2011 and Neijssel et al. 2021 for the case of Cygnus X-1), and the remnant mass and natal kick of the remaining supernova.

Inoue et al. (2016) and Finke and Razzaque (2017) use observations of ultraluminous X-ray sources (ULXs) with X-ray luminosities exceeding $10^{39} \mathrm{erg} \mathrm{s}^{-1}$ to estimate $\mathrm{BH}-\mathrm{BH}$ merger rates of $\lesssim 100 \mathrm{Gpc}^{-3} \mathrm{yr}^{-1}$ and $15-400 \mathrm{Gpc}^{-3} \mathrm{yr}^{-1}$, respectively. These estimates, however, are highly uncertain. They rely on the assumption that all ULXs are powered by accretion onto a $\mathrm{BH}$, which is contradicted by observations that at least some ULXs (perhaps all with confidently identified compact objects?) are powered by accretion onto an NS (Bachetti et al. 2014). They also make simplifying assumptions about the future evolutionary history of ULXs.

\subsection{Other observational constraints}

Abadie (2010) suggested that a strict upper limit of $<5 \times 10^{4}$ mergers $\mathrm{Gpc}^{-3} \mathrm{yr}^{-1}$ could be placed on the NS-NS merger rate by assuming that at least one companion must have been formed through a type Ib/Ic supernova (taking an optimistic rate estimate from Cappellaro et al. 1999). However, this upper limit is likely a very significant over-estimate, as most type Ib/Ic supernovae happen in other systems 
than merging binary neutron stars (perhaps even in systems that had already been disrupted, Hirai et al. 2020). At the same time, the type Ib/Ic supernova rate cannot even be considered a strict upper limit, because many merging neutron star binaries could form through a combination of electron-capture supernovae and ultra-stripped supernovae rather than classical core-collapse supernovae (Podsiadlowski et al. 2004; Dall'Osso et al. 2014; Tauris et al. 2015, 2017; Vigna-Gómez et al. 2018), and some compact remnants may form in the absence of any explosions.

Signatures of chemical enrichment associated with binary neutron star mergers, particularly the evolution of r-process nucleosynthesis elements, could serve as a useful constraint on the binary neutron star merger rate (Mennekens and Vanbeveren 2014; Hotokezaka et al. 2015; Beniamini et al. 2016). However, Siegel et al. (2019), Kobayashi et al. (2020) propose that magneto-rotational supernovae could instead be responsible for r-process enrichment.

Compact-object mergers or the subsequent evolution of compact-object merger products have been proposed as a source for some fast radio bursts (Totani 2013; Ravi and Lasky 2014); however, there are likely multiple progenitor channels for fast radio bursts (Pleunis et al. 2021), so these cannot presently be confidently used for merger rate estimates.

\section{Modelled rates}

In this section, we summarise some of the ongoing efforts to model the rates of compact object mergers formed through a variety of evolutionary channels. These channels offer alternative pathways to overcoming a key challenge in gravitationalwave astronomy. Gravitational-wave emission is only efficient in close binaries: in order for a circular binary with equal-mass components of mass $M$ to merge within a time $T$ exclusively through radiation reaction from gravitational-wave emission, the separation $a$ must be smaller than

$$
a \lesssim 3.7 R_{\odot}\left(\frac{M}{M_{\odot}}\right)^{3 / 4}\left(\frac{T}{14 \mathrm{Gyr}}\right)^{1 / 4} .
$$

Thus, to merge within the current age of the Universe, $14 \mathrm{Gyr}$, two $1.4 M_{\odot}$ neutron stars must be fewer than 5 solar radii apart, while even two $35 M_{\odot}$ black holes must be within a quarter of an astronomical unit.

On the other hand, massive stars typically expand to several au (hundreds to thousands of solar radii; 1 au $\approx 215 R_{\odot}$ ) in size during their evolution. Therefore, the key challenge to forming a merging compact-object binary lies in fitting a peg into a hole that is one or two orders of magnitude smaller than the size of the peg. The proposed solutions generally take the form of either relying on stellar evolution and mass transfer to overcome the problem in an isolated binary, or circumventing the problem altogether by relying on dynamics to form binaries with a sufficiently small separation that gravitational-wave emission can drive them to merge. Here we sketch out the key steps in the proposed formation channels, undertake back-of-theenvelope estimates of the rates, following Mandel and Farmer (2018), and describe 
some of the key uncertainties leading to the typically broad range of model predictions.

One particular source of uncertainty that is common to all models described below is the uncertain rate and metallicity of star formation across cosmic history. Even binaries merging in the relatively local Universe (detections through the third LIGO/Virgo observing run had moderate redshifts $z \lesssim 1$, Abbott et al. 2021d) could have formed at high redshift (Belczynski et al. 2016). Therefore, the rate of star formation at higher redshift influences local merger rates. Moreover, the yield of coalescing compact objects per unit star forming mass depends strongly on metallicity (Dominik et al. 2013; Neijssel et al. 2019). This is particularly true for black holes, whose massive progenitors can lose large fractions of their mass in winds in high-metallicity environments, widening the binaries and reducing merger rates. Therefore, the local merger rate is sensitive to the poorly known metallicityspecific star formation rate at high redshifts (Belczynski et al. 2010; Mapelli et al. 2017; Klencki et al. 2018; Chruslinska et al. 2019; Neijssel et al. 2019; Chruslinska and Nelemans 2019; Tang et al. 2020; Briel et al., 2021; Broekgaarden et al. 2021a, b; Santoliquido et al. 2021; van Son et al. 2021).

\subsection{Isolated binary evolution modelling (population synthesis)}

Almost all stars massive enough to form neutron stars and black holes are born in binaries or higher-multiplicity systems (Sana et al. 2012; Moe and Di Stefano 2017). We begin by considering compact-object coalescences in isolated binaries, namely those which do not appreciably interact with their environment. This channel has been studied since the 1970s. Some of the ground-breaking works particularly relevant to the formation of coalescing compact-object binaries include (Tutukov and Yungelson 1973; van den Heuvel and De Loore 1973; De Loore et al. 1975; Flannery and van den Heuvel 1975; Tutukov and Yungelson 1993; Lipunov et al. 1997; Bethe and Brown 1998; Dewi et al. 2006; Nelemans et al. 2001; Voss and Tauris 2003; Kalogera et al. 2007; O'Shaughnessy et al. 2008) (see Mandel and Farmer 2018 for a brief summary of the early history).

\subsubsection{Isolated binary evolution with mass transfer}

In this channel, two massive stars are born in a wide binary, giving them sufficient space to evolve. The binary shrinks as a consequence of mass transfer just when the stars themselves shrink in radius, allowing gravitational waves to take over.

We begin by sketching out a very simplified version of binary evolution in this model. The primary - the initially more massive star-completes core hydrogen fusion first. At the end of the main sequence, its helium-rich core contracts and the hydrogen-rich envelope expands. As the star fills the so-called Roche lobe, tidal gravity from the companion overcomes the self-gravity of the primary, and mass transfer onto the secondary commences. Eventually, the primary leaves behind a naked helium star, which continues nuclear fusion, losing mass through winds, until it collapses into a compact object. The mass loss and/or the natal kick from asymmetric mass ejection accompanying the supernova may unbind the binary. If it 
does not, the binary continues its evolution until the secondary expands and commences mass transfer onto the now compact-object primary. Because of the mass gain of the secondary during the first episode of mass transfer and the mass loss of the primary due to mass transfer, winds, and the supernova, the secondary may be significantly more massive than the primary at this stage. Consequently, mass transfer could harden the binary (van den Heuvel et al. 2017), and could even become dynamically unstable, leading to the formation of a common envelope (Paczyński 1976). Once the common envelope is not co-rotating with the binary, drag forces dissipate orbital energy, which may allow for common-envelope ejection after the binary hardens by two or three orders of magnitude (Ivanova et al. 2013). At this stage, the secondary has lost its envelope to become a naked helium star, so it can fit into the tight binary. (It is also possible that both stars are evolved giants during the first mass transfer interaction, leading to the simultaneous ejection of both envelopes during a single common-envelope event, as described by Dewi et al. 2006.) After more mass loss through winds the secondary may also collapse into a compact object. Assuming the second supernova leaves behind a bound, compact binary, this binary will eventually merge by emitting gravitational waves.

We can crudely estimate the expected merger rate through this channel with a Drake-like equation describing the fraction of all binaries that go on to make merging double compact objects of a particular type (Mandel and Farmer 2018), with chosen values corresponding to binary black hole formation:

$$
\begin{aligned}
f_{\text {DCO }}= & f_{\text {primary }} \times f_{\text {secondary }} \times f_{\text {init sep }} \times f_{\text {survive SN1 }} \times f_{\text {CE }} \times f_{\text {survive SN2 }} \\
& \times f_{\text {merge }} \sim 0.001 \times 0.5 \times 0.5 \times 1 \times 0.1 \times 1 \times 0.2=5 \times 10^{-6} .
\end{aligned}
$$

Assuming a local star formation rate of $\sim 10^{7} M_{\odot} \mathrm{Gpc}^{-3} \mathrm{yr}^{-1}$ (Madau and Dickinson 2014) with an average mass of $\sim M_{\odot}$, a yield of $f_{\mathrm{DCO}}=5 \times 10^{-6}$ would lead to a compact-object merger rate of $\sim 50 \mathrm{Gpc}^{-3} \mathrm{yr}^{-1}$.

Here we describe the terms in this equation, which allows us to highlight the broad range of physics involved and to comment on the key uncertainties. In practice, most modern models rely on some form of population synthesis, where large numbers of stellar binaries are evolved in order to compute the yield, frequently by using simplified recipes to reduce computational cost (Postnov and Yungelson 2014; Han et al. 2020).

Stars with initial mass at zero age on the main sequence of roughly $\gtrsim 20 M_{\odot}$ can form a $\mathrm{BH}$, so the fraction of all stars drawn from the initial mass function that will have mass in this range is $f_{\text {primary }} \approx 0.001$. Meanwhile, for forming an NS, the zeroage main sequence mass should be roughly between 8 and $20 M_{\odot}$, so $f_{\text {primary }} \approx 0.002$. The mass ratio between secondary and primary is roughly uniformly distributed (Sana et al. 2012), so if the primary falls in the mass range of interest, roughly half the secondaries will do so, i.e., $f_{\text {secondary }} \approx 0.5$. The fraction of binaries with initial separations in the range to allow for the first episode of mass transfer from evolved donors is quite large, $f_{\text {initsep }} \approx 0.5$, given the significant amount of expansion during this phase and the uniform in the logarithm distribution of initial separations (Öpik 1924). 
The probabilities of the binary surviving the first and second supernovae without being disrupted, $f_{\text {survive SN1 }}$ and $f_{\text {survive SN2 }}$, are both close to 1 for the heavier black holes that may form through complete collapse with negligible natal kicks (Mirabel 2017, but see Repetto et al. 2012; Mandel 2016; Atri et al. 2019). However, the high typical natal kicks of neutron stars, of order $300 \mathrm{~km} \mathrm{~s}^{-1}$ (Hobbs et al. 2005; Verbunt et al. 2017), can disrupt $\gtrsim 95 \%$ of wide binaries in the first supernova (VignaGómez et al. 2018; Renzo et al. 2019). Reduced kicks in electron-capture supernova (Podsiadlowski et al. 2004; Gessner and Janka 2018) may protect some of the wide binaries from unbinding, increasing $f_{\text {survive SN1 }}$. By the time of the second supernova, the binary will have gone through a common envelope in this channel, hardening it and making disruption less likely; moreover, low-mass helium star progenitors of neutron stars may re-expand after the helium main sequence, engage in another mass transfer episode (case BB mass transfer) and ultimately explode in very weak ultra-stripped supernovae with greatly reduced kicks (Tauris et al. 2015, but see Mandel and Müller 2020). Therefore, it is plausible that $f_{\text {survive SN2 }} \approx 1$, even for double neutron stars, although observed eccentricities of $\sim 0.6$ for a subset of Galactic double neutron stars, including the Hulse-Taylor binary, could point to a subpopulation of systems that avoid ultra-stripping and may be disrupted by the second supernova.

The common-envelope phase is perhaps the least certain one in massive binary evolution (Dominik et al. 2012). There are questions about both the onset of the common-envelope phase (do the mass ratio and the evolutionary state of the donor and accretor make mass transfer dynamically unstable?) and about the ultimate outcome of the evolution (is sufficient energy deposited in the envelope to eject it, or does this episode end in merger?). Typical population synthesis models based on current, very simplified recipes for both the stability mass transfer and the survivability of the common-envelope phase yield $f_{\mathrm{CE}} \approx 0.1$.

The fraction of compact-object binaries that ultimately coalesce in the age of the Universe $f_{\text {merge }}$ is set by the distribution of masses and separations. If the postcommon-envelope binary separation is distributed uniformly in the logarithm, the delay time $\tau$ between formation and merger, which scales with the fourth power of the separation, will also follow a log-uniform distribution, i.e., $p(\tau) \propto 1 / \tau$. In that case, the logarithmic distribution allows for typical $f_{\text {merge }} \approx 0.2$; however, as mentioned above, this can be significantly reduced, e.g., if winds increase the $\mathrm{BH}$ binary separation at high metallicity.

We now describe the key uncertainties in these estimates. Different treatments of some of the physics summarised below are responsible for the broad range of predictions reported in the executive summary.

The first significant uncertainty lies in the distribution of initial conditions: the masses of the two companions, their separations, and the binary eccentricity (Sana et al. 2012; Moe and Di Stefano 2017). These alone can impact the merger rate at the level of a factor of $\sim 6$ due to uncertainty in the initial mass function (Kroupa 2002) and by up to a factor of 2-3 due to uncertainty in the other parameters (de Mink and Belczynski 2015; Klencki et al. 2018). 
The treatment of mass transfer represents a key source of uncertainty in isolated binary evolution (Belczynski et al. 2022). Is the mass transfer dynamically stable? Stability thresholds can be formulated in terms of the mass ratio (Claeys et al. 2014) or the relative radial response of the star and its Roche lobe to mass transfer (Soberman et al. 1997; Ge et al. 2015). The depth of the convective envelope of the donor may play a critical role (Klencki et al. 2021). If the mass transfer is dynamically stable, how conservative is it, i.e., what fraction of mass lost by the donor does the companion accrete (e.g., Kippenhahn and Meyer-Hofmeister 1977), including the case of accretion onto compact objects (e.g., van Son et al. 2020)? And how much angular momentum is carried away by any mass that leaves the system, changing the orbital separation of the binary (e.g., Vinciguerra et al. 2020)? How does mass transfer in eccentric binaries affect their orbit (e.g.,. Sepinsky et al. 2010; Dosopoulou and Kalogera 2016)? If the mass transfer is dynamically unstable, leading to the formation of a common envelope, can the envelope be ejected, and what sets the orbital separation after the envelope ejection? Simple energy scalings are typically used to treat the common-envelope phase of binary evolution (Webbink 1984; de Kool 1990), partly because, despite decades of modelling common envelopes (Ivanova et al. 2013), the first incomplete models of this phase for massive stars are only just appearing (Ricker et al. 2019; Law-Smith et al. 2020; Lau et al. 2021). Last but not least, how does the history of mass transfer impact the final outcomes of stellar evolution, including supernova explosions (e.g., Brown et al. 2001; Schneider et al. 2021)?

A range of uncertainties in single stellar evolution play a key role. How much massive stars expand at various stages in their lives determines the onset and outcomes of mass transfer (e.g., Laplace et al. 2020). Stellar winds affect the final masses and separations (Vink 2017). The efficiency of tidal coupling determines orbital circularisation (Zahn 1977; Hut 1981; Vick and Lai 2018). The amount of mass lost in supernovae and the natal kicks that supernova remnants receive impact binary survival during the stellar explosions (Sipior and Sigurdsson 2002; Fryer et al. 2012; Müller 2020). Even relatively rare supernova variants such as electroncapture supernovae and (pulsational) pair-instability supernovae are important for coalescing compact object formation (e.g., Belczynski et al. 2018b; Stevenson et al. 2019; Farmer et al. 2020).

\subsubsection{Isolated binaries: chemically homogeneous evolution and population III stars}

One way to avoid the problem of fitting very extended stars into a binary tight enough to merge through gravitational waves is to prevent the stars from expanding in the first place. Here we consider two magic wands that might prevent expansion: efficient mixing or initially metal-free composition.

Suppose the stars are sufficiently massive, their metallicity is low enough to suppress strong winds, and they are close enough to be tidally locked in an orbit that enforces rotation at a significant fraction of the break-up velocity. Then the formation of temperature gradients between the poles and equator of the star may lead to large-scale circulation and efficient mixing (Eddington 1925; Sweet 1950; Endal and Sofia 1978). The mixing of helium out of the core into the envelope and 
fresh hydrogen from the envelope into the core allows the star to evolve chemically homogeneously, fusing almost all of the hydrogen into helium on the main sequence (Heger et al. 2000; Maeder and Meynet 2000; Yoon et al. 2006). After the end of hydrogen fusion, the entire star contracts into a naked helium star. Such systems avoid mass transfer after the main sequence, and may form heavy, ultimately coalescing black hole binaries in situ (Mandel and de Mink 2016; Marchant et al. 2016; de Mink and Mandel 2016). The high mass required for chemically homogeneous evolution makes this channel relevant only for the formation of merging black holes and, perhaps, rare NS-BH binaries (Marchant et al. 2017).

We can write a similar Drake's equation to Eq. (2) for the chemically homogeneous evolution channel. Following Mandel and de Mink (2016), the fraction of binaries that form coalescing binary black holes through this channel is

$$
\begin{aligned}
f_{\mathrm{BH}-\mathrm{BH}} & =f_{\text {primary }} \times f_{\text {secondary }} \times f_{\text {init sep }} \times f_{Z} \times f_{\text {merge }} \\
& \sim 0.0002 \times 0.5 \times 0.1 \times 0.1 \times 1=10^{-6} .
\end{aligned}
$$

Only the most massive stars with initial masses above $\sim 50 M_{\odot}$ are likely to experience sufficient mixing to evolve chemically homogeneously, lowering $f_{\text {primary }}$ to $\approx 2 \times 10^{-4}$. A flat mass ratio distribution would again yield $f_{\text {secondary }} \approx 0.5$. The range of initial separations allowing for chemically homogeneous evolution is at most a factor of two: stars that are initially too far apart either avoid tidal locking or do not rotate sufficiently rapidly, while stars that are too close overflow the L2 Lagrange point and promptly merge. If initial separations cover some 5 orders of magnitude and a uniform in the logarithm distribution of separations is assumed, up to $f_{\text {init sep }} \approx 0.1$ of binaries with the right mass range could still experience chemically homogeneous evolution. However, this also requires a sufficiently low metallicity, in part to avoid binary widening through excessive mass loss in winds, necessitating a factor describing the fraction of star formation at metallicities of interest, estimated here as $f_{Z} \approx 0.1$. On the other hand, if a binary evolves chemically homogeneously and forms black holes in situ, it is likely to be sufficiently compact to merge within the age of the Universe through gravitational-wave emission, so $f_{\text {merge }} \approx 1$. The overall yield estimate of $f_{\mathrm{BH}-\mathrm{BH}} \approx 10^{-6}$ would suggest a binary black hole coalescence rate of $\sim 10 \mathrm{Gpc}^{-3} \mathrm{yr}^{-1}$ for this channel.

The chemically homogeneous evolution channel as described above does not involve mass transfer after the main sequence and hence avoids some of uncertainties discussed in the previous subsection. On the other hand, the very possibility of efficient chemical mixing is uncertain. The observational evidence ranges from tentative to contradictory (Almeida et al. 2015; Mandel and de Mink 2016; Abdul-Masih et al. 2021) and theoretical models of mixing differ in the predicted thresholds on its efficiency. Moreover, much of the parameter space in this channel likely requires over-contact binaries (Marchant et al. 2016; du Buisson et al. 2020; Riley et al. 2021), which bring extra modelling challenges and uncertainties. On the other hand, a star could be spun up to rapid rotation and chemically homogeneous evolution by mass accretion from the companion (Cantiello et al. 2007). This mode of quasi chemically homogeneous evolution 
could also make a significant contribution to high-mass $\mathrm{BH}-\mathrm{BH}$ mergers originating in low-metallicity environments (Eldridge and Stanway 2016).

First-generation metal-free stars in the early Universe, known as population III stars, may represent an alternative way to avoid radial expansion after the main sequence. The absence of primordial carbon, nitrogen and oxygen in these stars prevents the $\mathrm{CNO}$ cycle from operating, so that hydrogen fusion can proceed only through the pp-chain reaction. While the core becomes hot enough for helium produced in the core to form carbon through the triple-alpha reaction, eventually allowing CNO cycle hydrogen fusion to take over, this may not happen to the same extent in the hydrogen shell around the helium core after core hydrogen exhaustion. Therefore, population III stars may experience reduced radial expansion relative to more metal-rich stars (Marigo et al. 2001) and can avoid mass transfer and many of the complications of the classical isolated binary evolution channel. Moreover, this means that the hydrogen envelope of such stars remains tightly bound, and is unlikely to be ejected during the stellar collapse as would be the case for population I and II supergiants (Nadezhin 1980; Lovegrove and Woosley 2013; Fernández et al. 2018) allowing the star to retain most of its mass (Kinugawa et al. 2021), akin to chemically homogeneously evolving stars. This mass retention is further aided by the drastically reduced wind-driven mass loss at zero metallicity. Finally, the lack of metals has been conjectured to suppress the fragmentation of a giant molecular cloud during star formation, increasing the typical mass of population III stars and making these prime progenitor candidates for binary black hole coalescences (Belczynski et al. 2004; Kinugawa et al. 2014; Inayoshi et al. 2016, 2017).

The lack of observational evidence and challenges in theoretical models put many of these assumptions in doubt. There are conflicting models for the initial properties of population III binaries (Hirano et al. 2014; Stacy et al. 2016) which could drastically reduce the predicted merger rate (Hartwig et al. 2016; Belczynski et al. 2017), while the possibility of significant stellar expansion could remove this altogether as an independent channel.

\subsection{Dynamical formation}

Dynamical formation broadly encompasses compact object mergers in which gravitational interactions with other stars played a significant role in bringing the two merging objects closer. Dynamical formation can be broadly divided into several categories based on environment. Historically, the focus has been primarily on young star clusters and globular clusters, where dynamical formation has been explored by Sigurdsson and Hernquist (1993), Kulkarni et al. (1993), Portegies Zwart and McMillan (2000), O'Leary et al. (2006), Banerjee et al. (2010), Downing et al. (2011), Morscher et al. (2015) through a mix of numerical gravitational experiments and semi-analytical estimates. We illustrate the expectations for globular cluster dynamical formation with another Drake's equation before discussing other dynamical formation environments: galactic nuclei and hierarchical triple systems. 


\subsubsection{Young star clusters and globular clusters}

The compact objects in the cluster may or may not have started out in the same binary. In the classical version of dynamical formation in a dense stellar environment the black holes, being heavier than other cluster objects, sink toward the centre of the cluster through mass segregation (Spitzer 1969; Binney and Tremaine 2008). Once there, they readily form binaries or substitute into existing binaries through three-body interactions: in a binary-single interaction, the lightest object is most likely to be ejected, while the two heavier objects remain behind in a binary (Sigurdsson and Phinney 1993; Ginat and Perets 2021). If the binary is hard-i.e., if its orbital velocity is larger than the velocity dispersion for the case of comparable component and interloper masses - subsequent interactions will further harden the binary as the energy of the binary is shared with the interlopers. Eventually, the binary may harden enough for gravitational waves to take over, provided the interaction rate is sufficiently high and the binary is not prematurely ejected from the cluster. This suggests that the number of $\mathrm{BH}-\mathrm{BH}$ mergers per globular cluster is:

$$
\begin{aligned}
N_{\mathrm{BH}-\mathrm{BH}, \text { cluster }} & =N_{\text {stars }} \times f_{\mathrm{BH}} \times f_{\text {retain }} \times f_{\text {merge }} \\
& \sim 10^{6} \times 0.001 \times 0.2 \times 1=200 .
\end{aligned}
$$

Here, we focus on $\mathrm{BH}-\mathrm{BHs}$, because neutron stars, being lighter, are preferentially ejected during interactions, reducing their formation rate (Ye et al. 2020). We consider the heaviest globular clusters with $N_{\text {stars }} \approx 10^{6}$ stars. We optimistically assume that all $\gtrsim 20 M_{\odot}$ stars will form a $\mathrm{BH}$, ignoring the likelihood that natal kicks will eject some of the BHs from the cluster, whose escape velocity is only $\sim 50 \mathrm{~km} \mathrm{~s}^{-1}$. This sets $f_{\mathrm{BH}} \approx 0.001$. In order for a binary to merge through the emission of gravitational-waves within $10 \mathrm{Gyr}$, its orbital velocity must be $\sim 500 \mathrm{~km} \mathrm{~s}^{-1}$ (Peters 1964). This is almost two orders of magnitude larger than the typical velocity dispersion of $10 \mathrm{~km} \mathrm{~s}^{-1}$. Tens of strong binary-single scattering interactions, mostly with other black holes, will be necessary to harden one binary by two orders of magnitude (Quinlan 1996). Once the binary becomes sufficiently hard, the lightest of the three interacting objects will typically be ejected from the cluster, depleting the fraction of retained black holes by a factor of a few (Heggie and Hut 2003). We therefore set the fraction of black holes retained for possible mergers to $f_{\text {retain }} \approx 0.2$. Finally, we consider a cluster sufficiently dense (central BH density above $\sim 10^{5} \mathrm{pc}^{-3}$ ) that most of the BHs that avoid being ejected do succeed in merging within $10 \mathrm{Gyr}$, so that $f_{\text {merge }}=1$. Even with these optimistic assumptions, and allowing for a constant merger rate over the 10 Gyr lifetime of globular clusters, whose space density is $\sim 1 \mathrm{Mpc}^{-3}$, we estimate a maximum merger rate of $200 \times 10^{9} \mathrm{Gpc}^{-3} \times 10^{-10} \mathrm{yr}^{-1}=20 \mathrm{Gpc}^{-3} \mathrm{yr}^{-1}$.

This very crude estimate helps highlight some of the issues that may impact the event rates. What is the fraction of compact objects retained in clusters despite natal kicks? What were the initial properties (mass distribution, binary fraction, density, velocity dispersions) of globular clusters, before the $\gtrsim 10 \mathrm{Gyr}$ of evolution typical 
for the locally observed clusters (Fragione and Kocsis 2018)? What fraction of merger products avoid being ejected by gravitational-wave recoil kicks and can be reused for subsequent hierarchical mergers (Rodriguez et al. 2018; Gerosa and Fishbach 2021)? What role does ongoing stellar evolution, including BH growth through tidal disruption of stars and gas accretion (Vesperini et al. 2010), play in dynamical formation? While multiple approaches to modelling cluster gravitational dynamics (e.g., N-body and Monte Carlo models) are expected to yield consistent results, the range of predictions highlighted in the executive summary tables and figures illustrates the impact of different approaches to answering the questions posed in this paragraph.

\subsubsection{Nuclear star clusters}

Dynamical formation has also been explored in the context of nuclear clusters in the centres of galaxies. Miller and Lauburg (2009) and Antonini and Rasio (2016) pointed out that nuclear clusters in the absence of a massive black hole, which are characterised by deeper potentials and hence higher escape velocities than globular clusters, could be promising environments for $\mathrm{BH}-\mathrm{BH}$ dynamical formation. O'Leary et al. (2009), Tsang (2013), Hoang et al. (2020), Rasskazov and Kocsis (2019) explored direct gravitational-wave bremsstrahlung captures from 2-body scattering. Other potential contributions include the deposition of stellar-mass black holes near a massive black hole by the infall of a globular cluster (Arca-Sedda and Gualandris 2018). We quote a selection of the latest predictions for all of these channels in the executive summary.

Meanwhile, Bellovary et al. (2016), Bartos et al. (2017), Stone et al. (2017), McKernan et al. (2018) emphasised the important role that an accretion disk in an active galactic nucleus could play in enhancing the rate of binary black hole mergers. The rich physics of the deep potential well combined with gas interactions can yield new merger channels (Tagawa et al. 2020). Compact objects may be captured by the disk or directly form in the disk; in fact, gas may not even be strictly necessary for this, as vector resonant relaxation against the stellar background could naturally lead massive objects to settle into a dis (Szölgyén and Kocsis 2018). Migration traps have been suggested as breeding grounds for dynamical interactions that will enhance the $\mathrm{BH}+\mathrm{BH}$ merger rate (Bellovary et al. 2016; Yang et al. 2019; Secunda et al. 2019; Peng and Chen 2021), although there is robust debate in the literature about their efficacy (e.g., Pan and Yang 2021). Gas accretion may aid gravitational waves in driving binaries toward merger as well as grow the black hole mass. Meanwhile, the very high escape velocity in the vicinity of a supermassive black hole ensures that recoil kicks are unlikely to eject merger products, so hierarchical mergers should be more common than in other dynamical environments (Yang et al. 2019; Secunda et al. 2020; Tagawa et al. 2021). The rich physics of active galactic nuclei make this channel both promising and complex, with many details such as the feedback from BH-gas interaction and accretion remaining a topic of ongoing work, leading to a broad range of merger rate predictions (Tagawa et al. 2020). 


\subsubsection{Hierarchical 3-body systems}

Another notable dynamical formation channel involves hierarchical triple systems (see Naoz 2016 for a review). In these systems, the transfer of angular momentum back and forth between the inner binary and the wider outer orbit of the third companion can cyclically increase the eccentricity of the inner binary (Lidov 1962; Kozai 1962). Once the eccentricity of the inner binary becomes sufficiently high, energy can be dissipated by gravitational waves or tides. This could drive the inner binary to merger (e.g., Wen 2003; Liu and Lai 2018). This channel may operate in a number of regimes, from isolated stellar triples (Silsbee and Tremaine 2017) to dynamically formed triples in clusters (Antonini et al. 2016) to triples in which the outer companion is a massive black hole in a galactic center (Antonini and Perets 2012; Hoang et al. 2018).

Unlike other dynamical formation scenarios, which are most promising for $\mathrm{BH}-$ BH mergers, hierarchical triples could be important for NS-NS formation, although predictions are sensitive to assumed NS natal kicks (Hamers and Thompson 2019). Extensions to the triple channel include higher multiplicity systems, such as quadruples (Fragione et al. 2020; Hamers et al. 2021; Vynatheya and Hamers 2021). Some authors have associated specific gravitational-wave sources, such as GW190814, with formation in hierarchical triples (e.g., Lu et al. 2021).

The triple channel can also operate in conjunction with other channels. For example, stellar evolution and associated mass loss could force a previously stable stellar triple into instability or drive the inner binary toward merger (Perets and Kratter 2012; Shappee and Thompson 2013; Stephan et al. 2016), while chemically homogenous evolution and triple dynamics could produce sequential BH-BH mergers (Vigna-Gómez et al. 2021b). In fact, it is the combination of triple dynamics with stellar evolution, mass transfer, tides and gravitational waves (as well as the possibility of other dynamical interactions with external perturbers) which makes it particularly challenging to analyse. The uncertain initial conditions, such as a possible correlation between the mutual inclination, separations and eccentricities of the inner and outer binaries (Moe and Di Stefano 2017), query the efficacy of this channel (Toonen et al. 2020) (but see Rose et al. 2019, who find that the observed orbits of massive stars may be consistent with being driven by triple dynamics).

\subsection{Exotica}

So far, we focussed exclusively on black holes of astrophysical origin, rather than primordial black holes forming from the collapse of early-Universe perturbations. The latter are potentially very interesting sources of gravitational waves. Bird et al. (2016), Ali-Haïmoud et al. (2017), Chen and Huang (2018) have argued that a significant fraction of dark matter could be contained in black holes with masses of tens of solar masses, and their mergers could be responsible for some of the gravitational-wave signals observed to date. However, even allowing for a significant amount of mass in primordial black holes, merger rate predictions are sensitive assumptions about the initial distribution of such sources in binaries or 
small clusters (e.g., Ali-Haïmoud et al. 2017; Korol et al. 2020; De Luca et al. 2020a) as well as possible accretion onto primordial black holes (De Luca et al. 2020b). Meanwhile, some of the scenarios proposed for the formation of BH-BH binaries involve physics beyond the standard model (Sakstein et al. 2020) and cosmological coupling (Croker et al. 2021).

The discovery of the BH-BH merger GW190521, which left behind a $\sim 150 M_{\odot}$ remnant (Abbott et al. 2020c), naturally renews interest in the possibility of intermediate mass black hole (IMBH) mergers. IMBH mergers may be a natural consequence of binary or dynamical evolution (e.g., Amaro-Seoane and Santamaría 2010; McKernan et al. 2012; Belczynski et al. 2014), and the gravitational waves from mergers of few hundred solar-mass IMBHs could be detected by current instruments (Veitch et al. 2015; Graff et al. 2015; Abbott et al. 2017d). Alternatively, stellar-mass compact objects could spiral into IMBHs in clusters. These intermediate-mass-ratio inspirals are also potentially detectable as gravitationalwave signals (Mandel et al. 2008; Haster et al. 2016b, a). However, the prevalence of IMBHs remains highly uncertain despite a variety of approaches to their detection (e.g., Pasham et al. 2014; Paynter et al. 2021). The challenges associated with observing IMBHs are discussed by Miller and Colbert (2004) and Greene et al. (2020). Given the many orders of magnitude uncertainties, we resist the temptation to review the merger rates of binaries involving IMBHs. Similarly, we do not specifically discuss hierarchical mergers of stellar-mass black holes or IMBHs as a channel for forming supermassive back holes at high redshifts (e.g., Volonteri 2010; Kroupa et al. 2020). The heaviest merging binaries observed through gravitational waves could represent the tail end of this process of hierarchical massive black-hole formation associated with mergers of ultra-dwarf galaxies (Palmese and Conselice 2021), and many more such mergers may be observable with third-generation gravitational-wave detectors (Gair et al. 2009).

\section{Outlook}

We reviewed the current estimates of compact-binary merger rates, including both direct observational constraints and theoretical models motivated by observations. We highlighted some of the key sources of uncertainty in both observations and models. Of course, these uncertainties can be alternatively viewed as opportunities.

The number of observations is growing rapidly. The number of direct gravitational-wave detections has grown from 0 to 50 between 2015 and 2020 . The range, the number of observed sources, and the measurement accuracy will all increase as the detector sensitivity improves (Abbott et al. 2020b). Eventually, third-generation gravitational-wave instruments will be able to precisely probe the evolving compact binary merger rate and mass distribution across cosmic time (e.g., Kalogera et al. 2019; $\mathrm{Ng}$ et al. 2021). The number and quality of electromagnetic observations are also rapidly growing, again with a very positive outlook, e.g., for kilonovae as new instruments like the Vera C. Rubin Observatory come online (e.g., Chase et al. 2021). 
These improvements in observations are being matched by a growing sophistication in the models. On the one hand, increasingly complex models of binary evolution and dynamics are sensitive to a broad range of uncertain assumptions about initial conditions, stellar evolution, mass loss, mass transfer, supernova kicks, and the cosmic star formation history of the Universe. On the other hand, there is growing evidence that observations of the rates and properties of compact-object mergers, coupled with other observational constraints, will make it possible to resolve these uncertainties and come away with a deep understanding of the physics of compact binary formation (e.g., Mandel and O'Shaughnessy 2010; Stevenson et al. 2015; Barrett et al. 2018; Wong et al. 2021).

While we focussed on overall rate estimates in this review, specific formation channels are likely to carry distinguishable observational signatures. We mention two predictions for merging binary black holes by way of example. Dynamically formed binary black holes should have isotropically oriented spin directions, while $\mathrm{BH}-\mathrm{BH}$ mergers from isolated binary evolution are expected to have spins preferentially aligned with the orbital angular momentum (e.g., Farr et al. 2017; Rodriguez et al. 2016b). Is there already evidence for a separate population of dynamically formed systems based on spin-orbit misalignment angles (Abbott et al. 2021c; Roulet et al. 2021; Galaudage et al. 2021)? Meanwhile, the orbits of most binary black holes are expected to circularise through gravitational-wave emission by the time they approach merger and become detectable with current instruments (Peters 1964). But a $\sim 5 \%$ fraction of binaries that evolve through binary-single dynamical scatterings may buck the trend, becoming captured at very high eccentricities and small separations through gravitational-wave bremsstrahlung during chaotic interactions (Samsing et al. 2014; Rodriguez et al. 2018). Could the tentative evidence for eccentric binary black holes in gravitational-wave data (Romero-Shaw et al. 2021) lead to an inferred measurement of the rate of dynamically formed binaries (Zevin et al. 2021)?

Reviewing such a rapidly developing field presents obvious challenges, and we are aware that we have only shown a selection of highlights. We hope that this is still a useful snapshot of the status of the field against which future rapid progress can be compared. In fact, we find ourselves in the awkward position of wishing for this review to become obsolete soon after it is written, to be updated as the field moves forward!

Supplementary Information The online version contains supplementary material available at https://doi. org/10.1007/s41114-021-00034-3.

Acknowledgements We are grateful to Iminhaji Ablimit, Manuel Arca Sedda, Chris Belczynski, Edo Berger, Alison Farmer, Saavik Ford, Gabriele Franciolini, Hongwei Ge, Adrian Hamers, Carl-Johan Haster, Ryosuke Hirai, Griffin Hosseinzadeh, Stephen Justham, Bence Kocsis, Pavel Kroupa, Boyuan Liu, Wenbin Lu, Michela Mapelli, Barry McKernan, Selma de Mink, Alex Nitz, Mathieu Renzo, Smadar Naoz, Carl Rodriguez, Navin Sridhar, Thomas Tauris, Ed van den Heuvel, Salvatore Vitale, Tom Wagg, Lev Yungelson, Michael Zevin, and members of Team COMPAS and the WERRD group for discussions and suggestions. IM carried out parts of this work at the Aspen Center for Physics, which is supported by National Science Foundation grant PHY-1607611, and at MIAPP. IM acknowledges support from the Australian Research Council Centre of Excellence for Gravitational Wave Discovery (OzGrav), through project number CE17010004. IM is a recipient of the Australian Research Council Future Fellowship FT190100574. 
Open Access This article is licensed under a Creative Commons Attribution 4.0 International License, which permits use, sharing, adaptation, distribution and reproduction in any medium or format, as long as you give appropriate credit to the original author(s) and the source, provide a link to the Creative Commons licence, and indicate if changes were made. The images or other third party material in this article are included in the article's Creative Commons licence, unless indicated otherwise in a credit line to the material. If material is not included in the article's Creative Commons licence and your intended use is not permitted by statutory regulation or exceeds the permitted use, you will need to obtain permission directly from the copyright holder. To view a copy of this licence, visit http:// creativecommons.org/licenses/by/4.0/.

Data availability The collated data and code to reproduce all figures in this review are publicly available through Broekgaarden and Mandel (2021).

\section{References}

Aasi J, Abbott BP, Abbott R, Abbott T, Abernathy MR, Ackley K, Adams C, Adams T, Addesso P et al (2015) Advanced LIGO. Class Quantum Grav 32(7):074001. https://doi.org/10.1088/0264-9381/32/ 7/074001. arXiv:1411.4547 [gr-qc]

Abadie $\mathbf{J}$ et al (2010) Predictions for the rates of compact binary coalescences observable by groundbased gravitational-wave detectors. Class Quantum Grav 27(17):173001. https://doi.org/10.1088/ 0264-9381/27/17/173001. arXiv:1003.2480 [astro-ph.HE]

Abbott BP, Abbott R, Abbott TD, Abernathy MR, Acernese F, Ackley K, Adams C, Adams T, Addesso P, Adhikari RX et al (2016) GW150914: implications for the stochastic gravitational-wave background from binary black holes. Phys Rev Lett 116(13):131102. https://doi.org/10.1103/PhysRevLett.116. 131102. arXiv:1602.03847 [gr-qc]

Abbott BP, Abbott R, Abbott TD, Abernathy MR, Acernese F, Ackley K, Adams C, Adams T, Addesso P, Adhikari RX et al (2016) Observation of gravitational waves from a binary black hole merger. Phys Rev Lett 116(6):061102. https://doi.org/10.1103/PhysRevLett.116.061102. arXiv:1602.03837 [grqc]

Abbott BP, Abbott R, Abbott TD, Acernese F, Ackley K, Adams C, Adams T, Addesso P, Adhikari RX, Adya VB et al (2017) Gravitational waves and gamma-rays from a binary neutron star merger: GW170817 and GRB 170817A. Astrophys J Lett 848:L13. https://doi.org/10.3847/2041-8213/ aa920c. arXiv: 1710.05834 [astro-ph.HE]

Abbott BP, Abbott R, Abbott TD, Acernese F, Ackley K, Adams C, Adams T, Addesso P, Adhikari RX, Adya VB et al (2017) GW170817: observation of gravitational waves from a binary neutron star inspiral. Phys Rev Lett 119(16):161101. https://doi.org/10.1103/PhysRevLett.119.161101. arXiv: 1710.05832 [gr-qc]

Abbott BP, Abbott R, Abbott TD, Acernese F, Ackley K, Adams C, Adams T, Addesso P, Adhikari RX, Adya VB et al (2017) Multi-messenger observations of a binary neutron star merger. Astrophys J Lett 848:L12. https://doi.org/10.3847/2041-8213/aa91c9. arXiv:1710.05833 [astro-ph.HE]

Abbott BP, Abbott R, Abbott TD, Acernese F, Ackley K, Adams C, Adams T, Addesso P, Adhikari RX, Adya VB et al (2017) Search for intermediate mass black hole binaries in the first observing run of Advanced LIGO. Phys Rev D 96(2):022001. https://doi.org/10.1103/PhysRevD.96.022001. arXiv: 1704.04628 [gr-qc]

Abbott BP, Abbott R, Abbott TD, Abraham S, Acernese F, Ackley K, Adams C et al (2019) Binary black hole population properties inferred from the first and second observing runs of advanced ligo and advanced virgo. Astrophys J Lett 882(2):L24. https://doi.org/10.3847/2041-8213/ab3800. arXiv: 1811.12940 [astro-ph.HE]

Abbott BP, Abbott R, Abbott TD, Abraham S, Acernese F, Ackley K, Adams C, Adhikari RX, Adya VB, Affeldt C et al (2020) GW190425: observation of a compact binary coalescence with total mass $\sim 3.4 \mathrm{M}_{\odot}$. Astrophys J Lett 892(1):L3. https://doi.org/10.3847/2041-8213/ab75f5. arXiv:2001. 01761 [astro-ph.HE] 
Abbott BP, Abbott R, Abbott TD, Abraham S, Acernese F, Ackley K, Adams C, Adya VB, Affeldt C, Agathos $\mathrm{M}$ et al (2020) Prospects for observing and localizing gravitational-wave transients with advanced LIGO, advanced Virgo and KAGRA. Living Rev Relativ 23:3. https://doi.org/10.1007/ s41114-020-00026-9

Abbott BP et al (2019) GWTC-1: a gravitational-wave transient catalog of compact binary mergers observed by LIGO and Virgo during the first and second observing runs. Phys Rev X 9(3):031040. https://doi.org/10.1103/PhysRevX.9.031040. arXiv:1811.12907 [astro-ph.HE]

Abbott R, Abbott TD, Abraham S, Acernese F et al (2020) GW190521: a binary black hole merger with a total mass of 150 Msun. Phys Rev Lett 125(10):101102. https://doi.org/10.1103/PhysRevLett.125. 101102. arXiv:2009.01075 [gr-qc]

Abbott R, Abbott TD, Abraham S, Acernese F et al (2020) GW190814: gravitational waves from the coalescence of a 23 solar mass black hole with a 2.6 solar mass compact object. Astrophys J Lett 896(2):L44. https://doi.org/10.3847/2041-8213/ab960f

Abbott R, Abbott TD, Abraham S, Acernese F, Ackley K et al (2021) Observation of gravitational waves from two neutron star-black hole coalescences. Astrophys J Lett 915(1):L5. https://doi.org/10.3847/ 2041-8213/ac082e. arXiv:2106.15163 [astro-ph.HE]

Abbott R, Abbott TD, Abraham S, Acernese F, et al. (2021b) GWTC-2.1: deep extended catalog of compact binary coalescences observed by LIGO and Virgo during the first half of the third observing run. arXiv e-prints arXiv:2108.01045 [gr-qc]

Abbott R, Abbott TD, Abraham S, Acernese F et al (2021) Population properties of compact objects from the second LIGO-Virgo gravitational-wave transient catalog. Astrophys J Lett 913(1):L7. https://doi. org/10.3847/2041-8213/abe949. arXiv:2010.14533 [astro-ph.HE]

Abbott R, Abbott TD, Acernese F, Ackley K, Adams C, Adhikari N, et al. (2021d) GWTC-3: compact binary coalescences observed by LIGO and Virgo during the second part of the third observing run. arXiv e-prints arXiv:2111.03606 [gr-qc]

Abbott R, Abbott TD, Acernese F, Ackley K, Adams C, Adhikari N, et al. (2021e) The population of merging compact binaries inferred using gravitational waves through GWTC-3. arXiv e-prints arXiv:2111.03634 [astro-ph.HE]

Abdul-Masih M, Sana H, Hawcroft C, Almeida LA, Brands SA, de Mink SE, Justham S, Langer N, Mahy L, Marchant P, Menon A, Puls J, Sundqvist J (2021) Constraining the overcontact phase in massive binary evolution. I. Mixing in V382 Cyg, VFTS 352, and OGLE SMC-SC10 108086. Astron Astrophys 651:A96. https://doi.org/10.1051/0004-6361/202040195. arXiv:2104.07621 [astroph.SR]

Ablimit I, Maeda K (2018) Monte Carlo population synthesis on massive star binaries: astrophysical implications for gravitational-wave sources. Astrophys J 866(2):151. https://doi.org/10.3847/15384357/aae378. arXiv:1710.05504 [astro-ph.HE]

Adhikari RX, Ajith P, Chen Y, Clark JA, Dergachev V, Fotopoulos NV, Gossan SE, Mandel I, Okounkova M, Raymond V, Read JS (2019) Astrophysical science metrics for next-generation gravitational-wave detectors. Class Quantum Grav 36(24):245010. https://doi.org/10.1088/13616382/ab3cff. arXiv:1905.02842 [astro-ph.HE]

Ali-Haïmoud Y, Kovetz ED, Kamionkowski M (2017) Merger rate of primordial black-hole binaries. Phys Rev D 96(12):123523. https://doi.org/10.1103/PhysRevD.96.123523. arXiv:1709.06576 [astroph.CO]

Almeida LA, Sana H, de Mink SE, Tramper F, Soszyński I, Langer N, Barbá RH, Cantiello M, Damineli A, de Koter A, Garcia M, Gräfener G, Herrero A, Howarth I, Maíz Apellániz J, Norman C, RamírezAgudelo OH, Vink JS (2015) Discovery of the massive overcontact binary VFTS352: evidence for enhanced internal mixing. Astrophys J 812:102. https://doi.org/10.1088/0004-637X/812/2/102. arXiv:1509.08940 [astro-ph.SR]

Amaro-Seoane P, Santamaría L (2010) Detection of IMBHs with ground-based gravitational wave observatories: a biography of a binary of black holes, from birth to death. Astrophys J 722:1197-1206. https://doi.org/10.1088/0004-637X/722/2/1197. arXiv:0910.0254

Amaro-Seoane P, Audley H, Babak S, Baker J, Barausse E, Bender P, Berti E, Binetruy P, et al. (2017) Laser interferometer space antenna. ArXiv e-prints arXiv:1702.00786 [astro-ph.IM]

Andreoni I, Coughlin MW, Kool EC, Kasliwal MM, Kumar H, Bhalerao V, Sagués Carracedo A et al (2021) Fast-transient searches in real time with ZTFReST: identification of three optically discovered gamma-ray burst afterglows and new constraints on the kilonova rate. Astrophys $\mathbf{J}$ 918(2):63. https://doi.org/10.3847/1538-4357/ac0bc7. arXiv:2104.06352 [astro-ph.HE] 
Andrews JJ, Mandel I (2019) Double neutron star populations and formation channels. Astrophys J Lett 880:L8. https://doi.org/10.3847/2041-8213/ab2ed1. arXiv:1904.12745 [astro-ph.HE]

Antonini F, Gieles M (2020) Merger rate of black hole binaries from globular clusters: theoretical error bars and comparison to gravitational wave data from GWTC-2. Phys Rev D 102(12):123016. https:// doi.org/10.1103/PhysRevD.102.123016. arXiv:2009.01861 [astro-ph.HE]

Antonini F, Perets HB (2012) Secular evolution of compact binaries near massive black holes: gravitational wave sources and other exotica. Astrophys J 757(1):27. https://doi.org/10.1088/0004637X/757/1/27. arXiv:1203.2938 [astro-ph.GA]

Antonini F, Rasio FA (2016) Merging black hole binaries in galactic nuclei: implications for advancedLIGO detections. Astrophys J 831:187. https://doi.org/10.3847/0004-637X/831/2/187. arXiv:1606. 04889 [astro-ph.HE]

Antonini F, Chatterjee S, Rodriguez CL, Morscher M, Pattabiraman B, Kalogera V, Rasio FA (2016) Black hole mergers and blue stragglers from hierarchical triples formed in globular clusters. Astrophys J 816:65. https://doi.org/10.3847/0004-637X/816/2/65. arXiv:1509.05080

Antonini F, Toonen S, Hamers AS (2017) Binary black hole mergers from field triples: properties, rates, and the impact of stellar evolution. Astrophys J 841(2):77. https://doi.org/10.3847/1538-4357/ aa6f5e. arXiv:1703.06614 [astro-ph.GA]

Arca Sedda M (2020) Birth, life, and death of black hole binaries around supermassive black holes: dynamical evolution of gravitational wave sources. Astrophys J 891(1):47. https://doi.org/10.3847/ 1538-4357/ab723b. arXiv:2002.04037 [astro-ph.GA]

Arca Sedda M (2020) Dissecting the properties of neutron star-black hole mergers originating in dense star clusters. Commun Phys 3(1):43. https://doi.org/10.1038/s42005-020-0310-x. arXiv:2003.02279 [astro-ph.GA]

Arca Sedda M (2021) Dynamical formation of the GW190814 merger. Astrophys J Lett 908(2):L38. https://doi.org/10.3847/2041-8213/abdfcd. arXiv:2102.03364 [astro-ph.HE]

Arca-Sedda M, Gualandris A (2018) Gravitational wave sources from inspiralling globular clusters in the Galactic Centre and similar environments. Mon Not R Astron Soc 477(4):4423-4442. https://doi. org/10.1093/mnras/sty922. arXiv:1804.06116 [astro-ph.GA]

Artale MC, Mapelli M, Giacobbo N, Sabha NB, Spera M, Santoliquido F, Bressan A (2019) Host galaxies of merging compact objects: mass, star formation rate, metallicity, and colours. Mon Not R Astron Soc 487:1675-1688. https://doi.org/10.1093/mnras/stz1382. arXiv:1903.00083

Askar A, Szkudlarek M, Gondek-Rosińska D, Giersz M, Bulik T (2017) MOCCA-SURVEY Database - I. Coalescing binary black holes originating from globular clusters. Mon Not R Astron Soc 464:L36L40. https://doi.org/10.1093/mnrasl/slw177. arXiv:1608.02520 [astro-ph.HE]

Atri P, Miller-Jones JCA, Bahramian A, Plotkin RM, Jonker PG, Nelemans G et al (2019) Potential kick velocity distribution of black hole X-ray binaries and implications for natal kicks. Mon Not R Astron Soc 489(3):3116-3134. https://doi.org/10.1093/mnras/stz2335. arXiv:1908.07199 [astro-ph.HE]

Bachetti M, Harrison FA, Walton DJ, Grefenstette BW, Chakrabarty D, Fürst F et al (2014) An ultraluminous X-ray source powered by an accreting neutron star. Nature 514(7521):202-204. https://doi.org/10.1038/nature13791. arXiv:1410.3590 [astro-ph.HE]

Bae YB, Kim C, Lee HM (2014) Compact binaries ejected from globular clusters as gravitational wave sources. Mon Not R Astron Soc 440:2714-2725. https://doi.org/10.1093/mnras/stu381. arXiv:1308. 1641 [astro-ph.HE]

Baibhav V, Berti E, Gerosa D, Mapelli M, Giacobbo N, Bouffanais Y, Di Carlo UN (2019) Gravitationalwave detection rates for compact binaries formed in isolation: LIGO/Virgo O3 and beyond. Phys Rev D 100(6):064060. https://doi.org/10.1103/PhysRevD.100.064060. arXiv:1906.04197 [gr-qc]

Banerjee S (2021) Stellar-mass black holes in young massive and open stellar clusters - V. Comparisons with LIGO-Virgo merger rate densities. Mon Not R Astron Soc 503(3):3371-3385. https://doi.org/ 10.1093/mnras/stab591. arXiv:2011.07000 [astro-ph.HE]

Banerjee S, Baumgardt H, Kroupa P (2010) Stellar-mass black holes in star clusters: implications for gravitational wave radiation. Mon Not R Astron Soc 402:371-380. https://doi.org/10.1111/j.13652966.2009.15880.x. arXiv:0910.3954

Barrett JW, Gaebel SM, Neijssel CJ, Vigna-Gómez A, Stevenson S, Berry CPL, Farr WM, Mandel I (2018) Accuracy of inference on the physics of binary evolution from gravitational-wave observations. Mon Not R Astron Soc 477:4685-4695. https://doi.org/10.1093/mnras/sty908. arXiv: 1711.06287 [astro-ph.HE] 
Bartos I, Kocsis B, Haiman Z, Márka S (2017) Rapid and bright stellar-mass binary black hole mergers in active galactic nuclei. Astrophys J 835:165. https://doi.org/10.3847/1538-4357/835/2/165. arXiv: 1602.03831 [astro-ph.HE]

Bavera SS, Fragos T, Zevin M, Berry CPL, Marchant P, Andrews JJ, Coughlin S, Dotter A, Kovlakas K, Misra D, Serra-Perez JG, Qin Y, Rocha KA, Román-Garza J, Tran NH, Zapartas E (2021) The impact of mass-transfer physics on the observable properties of field binary black hole populations. Astron Astrophys 647:A153. https://doi.org/10.1051/0004-6361/202039804. arXiv:2010.16333 [astro-ph.HE]

Belczynski K, Bulik T, Rudak B (2004) The first stellar binary black holes: the strongest gravitational wave burst sources. Astrophys J Lett 608:L45-L48. https://doi.org/10.1086/422172. arXiv:0403361 [astro-ph]

Belczynski K, Dominik M, Bulik T, O’Shaughnessy R, Fryer C, Holz DE (2010) The effect of metallicity on the detection prospects for gravitational waves. Astrophys J Lett 715:L138-L141. https://doi.org/ 10.1088/2041-8205/715/2/L138. arXiv:1004.0386 [astro-ph.HE]

Belczynski K, Bulik T, Bailyn C (2011) The fate of Cyg X-1: an empirical lower limit on black-holeneutron-star merger rate. Astrophys J Lett 742(1):L2. https://doi.org/10.1088/2041-8205/742/1/L2. arXiv:1107.4106 [astro-ph.GA]

Belczynski K, Bulik T, Mandel I, Sathyaprakash BS, Zdziarski AA, Mikołajewska J (2013) Cyg X-3: a galactic double black hole or black-hole-neutron-star progenitor. Astrophys J 764:96. https://doi.org/ 10.1088/0004-637X/764/1/96. arXiv:1209.2658 [astro-ph.HE]

Belczynski K, Buonanno A, Cantiello M, Fryer CL, Holz DE, Mandel I, Miller MC, Walczak M (2014) The formation and gravitational-wave detection of massive stellar black hole binaries. Astrophys $\mathrm{J}$ 789:120. https://doi.org/10.1088/0004-637X/789/2/120. arXiv:1403.0677 [astro-ph.HE]

Belczynski K, Holz DE, Bulik T, O'Shaughnessy R (2016) The first gravitational-wave source from the isolated evolution of two stars in the 40-100 solar mass range. Nature 534:512-515. https://doi.org/ 10.1038/nature18322. arXiv:1602.04531 [astro-ph.HE]

Belczynski K, Ryu T, Perna R, Berti E, Tanaka TL, Bulik T (2017) On the likelihood of detecting gravitational waves from Population III compact object binaries. Mon Not $\mathrm{R}$ Astron Soc 471(4):4702-4721. https://doi.org/10.1093/mnras/stx1759. arXiv:1612.01524 [astro-ph.HE]

Belczynski K, Askar A, Arca-Sedda M, Chruslinska M, Donnari M, Giersz M, Benacquista M, Spurzem R, Jin D, Wiktorowicz G, Belloni D (2018) The origin of the first neutron star-neutron star merger. Astron Astrophys 615:A91. https://doi.org/10.1051/0004-6361/201732428. arXiv:1712.00632 [astro-ph.HE]

Belczynski K, Bulik T, Olejak A, Chruslinska M, Singh N, Pol N, Zdunik L, O’Shaughnessy R, McLaughlin M, Lorimer D, Korobkin O, van den Heuvel EPJ, Davies MB, Holz DE (2018b) Binary neutron star formation and the origin of GW170817. arXiv e-prints arXiv:1812.10065 [astro-ph.HE]

Belczynski K, Klencki J, Fields CE, Olejak A, Berti E, Meynet G, Fryer CL, Holz DE, O’Shaughnessy R et al (2020) Evolutionary roads leading to low effective spins, high black hole masses, and O1/O2 rates for LIGO/Virgo binary black holes. Astron Astrophys 636:A104. https://doi.org/10.1051/00046361/201936528. arXiv:1706.07053 [astro-ph.HE]

Belczynski K, Romagnolo A, Olejak A, Klencki J, Chattopadhyay D, Stevenson S, Miller MC, Lasota JP, Crowther PA (2022) The uncertain future of massive binaries obscures the origin of LIGO/Virgo sources. Astrophys J 925:69. https://doi.org/10.3847/1538-4357/ac375a. arXiv:2108.10885 [astroph.HE]

Bellovary JM, Mac Low MM, McKernan B, Ford KES (2016) Migration traps in disks around supermassive black holes. Astrophys J Lett 819:L17. https://doi.org/10.3847/2041-8205/819/2/L17. arXiv: 1511.00005

Beniamini P, Hotokezaka K, Piran T (2016) r-process production sites as inferred from eu abundances in Dwarf galaxies. Astrophys J 832:149. https://doi.org/10.3847/0004-637X/832/2/149. arXiv:1608. 08650 [astro-ph.HE]

Berger E (2014) Short-duration gamma-ray bursts. Annu Rev Astron Astrophys 52:43-105. https://doi. org/10.1146/annurev-astro-081913-035926. arXiv:1311.2603 [astro-ph.HE]

Bethe HA, Brown GE (1998) Evolution of binary compact objects that merge. Astrophys J 506:780-789. https://doi.org/10.1086/306265. arXiv:9802084 [astro-ph]

Binney J, Tremaine S (2008) Galactic dynamics. Princeton series in astrophysics, 2nd edn. Princeton University Press, Princeton 
Bird S, Cholis I, Muñoz JB, Ali-Haïmoud Y, Kamionkowski M, Kovetz ED, Raccanelli A, Riess AG (2016) Did LIGO detect dark matter? Phys Rev Lett 116(20):201301. https://doi.org/10.1103/ PhysRevLett.116.201301. arXiv:1603.00464

Boco L, Lapi A, Goswami S, Perrotta F, Baccigalupi C, Danese L (2019) Merging rates of compact binaries in galaxies: perspectives for gravitational wave detections. Astrophys J 881(2):157. https:// doi.org/10.3847/1538-4357/ab328e. arXiv:1907.06841 [astro-ph.GA]

Briel MM, Eldridge JJ, Stanway ER, Stevance HF, Chrimes AA (2021) Estimating transient rates fromcosmological simulations and BPASS. arXiv e-prints arXiv:2111.08124 [astro-ph.CO]

Broekgaarden FS, Mandel I (2021) Data set from: rates of compact object coalescences. https://doi.org/ 10.5281 /zenodo.5847743, Version 4 has additional rates from recent papers and is updated to include the GWTC-3 results 11/19/21. This is the version that was used for the re-submitted paper. Version 3 has additional rates that we added after receiving useful feedback from the community (post ArXiv submission). Version 2 has updated and additional rates that we added. Version 1 of the data that was used for the first ArXiv submission

Broekgaarden FS, Berger E, Neijssel CJ, Vigna-Gómez A, Chattopadhyay D, Stevenson S, Chruslinska M, Justham S, de Mink SE, Mandel I (2021a) Impact of massive binary star and cosmic evolution on gravitational wave observations I: black hole-neutron star mergers. Mon Not R Astron Soc 508(4):5028-5063. https://doi.org/10.1093/mnras/stab2716. arXiv:2103.02608 [astro-ph.HE]

Broekgaarden FS, Berger E, Stevenson S, Justham S, Mandel I, Chruslinska M, van Son LAC, Wagg T,Vigna-Gómez A, de Mink SE, Chattopadhyay D, Neijssel CJ (2021b) Impact of Massive Binary Star andCosmic Evolution on Gravitational Wave Observations II: Double Compact Object Rates and Properties. arXive-prints arXiv:2112.05763 [astro-ph.HE]

Brown GE, Heger A, Langer N, Lee CH, Wellstein S, Bethe HA (2001) Formation of high mass X-ray black hole binaries. New Astron 6(7):457-470. https://doi.org/10.1016/S1384-1076(01)00077-X. arXiv:0102379 [astro-ph]

Bulik T, Belczynski K, Prestwich A (2011) IC10 X-1/NGC300 X-1: the very immediate progenitors of BH-BH binaries. Astrophys J 730:140. https://doi.org/10.1088/0004-637X/730/2/140. arXiv:0803. 3516

Cantiello M, Yoon SC, Langer N, Livio M (2007) Binary star progenitors of long gamma-ray bursts. Astron Astrophys 465:L29-L33. https://doi.org/10.1051/0004-6361:20077115. arXiv:0702540 [astro-ph]

Cappellaro E, Evans R, Turatto M (1999) A new determination of supernova rates and a comparison with indicators for galactic star formation. Astron Astrophys 351:459-466 arXiv:9904225 [astro-ph]

Chase EA, O'Connor B, Fryer CL, Troja E, Korobkin O, Wollaeger RT, Ristic M, Fontes CJ, Hungerford AL, Herring AM (2021) Kilonova detectability with wide-field instruments. arXiv e-prints arXiv: 2105.12268 [astro-ph.HE]

Chattopadhyay D, Stevenson S, Hurley JR, Bailes M, Broekgaarden F (2021) Modelling neutron starblack hole binaries: future pulsar surveys and gravitational wave detectors. Mon Not R Astron Soc 504(3):3682-3710. https://doi.org/10.1093/mnras/stab973. arXiv:2011.13503 [astro-ph.HE]

Chen ZC, Huang QG (2018) Merger rate distribution of primordial black hole binaries. Astrophys J 864(1):61. https://doi.org/10.3847/1538-4357/aad6e2. arXiv:1801.10327 [astro-ph.CO]

Choksi N, Volonteri M, Colpi M, Gnedin OY, Li H (2019) The star clusters that make black hole binaries across cosmic time. Astrophys J 873(1):100. https://doi.org/10.3847/1538-4357/aaffde. arXiv:1809. 01164 [astro-ph.GA]

Chruslinska M, Nelemans G (2019) Metallicity of stars formed throughout the cosmic history based on the observational properties of star forming galaxies. Mon Not R Astron Soc https://doi.org/10.1093/ mnras/stz2057. arXiv:1907.11243

Chruslinska M, Belczynski K, Klencki J, Benacquista M (2018) Double neutron stars: merger rates revisited. Mon Not R Astron Soc 474(3):2937-2958. https://doi.org/10.1093/mnras/stx2923. arXiv: 1708.07885 [astro-ph.HE]

Chruslinska M, Nelemans G, Belczynski K (2019) The influence of the distribution of cosmic star formation at different metallicities on the properties of merging double compact objects. Mon Not $\mathrm{R}$ Astron Soc 482:5012-5017. https://doi.org/10.1093/mnras/sty3087. arXiv:1811.03565 [astroph.HE]

Chu Q, Yu S, Lu Y (2021) Formation and evolution of binary neutron stars: mergers and their host galaxies. Mon Not R Astron Soc https://doi.org/10.1093/mnras/stab2882. arXiv:2110.04687 [astroph.GA] 
Claeys JSW, Pols OR, Izzard RG, Vink J, Verbunt FWM (2014) Theoretical uncertainties of the Type Ia supernova rate. Astron Astrophys 563:A83. https://doi.org/10.1051/0004-6361/201322714. arXiv: 1401.2895 [astro-ph.SR]

Clark JPA, van den Heuvel EPJ, Sutantyo W (1979) Formation of neutron star binaries and their importance for gravitational radiation. Astron Astrophys 72:120-128

Clausen D, Sigurdsson S, Chernoff DF (2013) Black hole-neutron star mergers in globular clusters. Mon Not R Astron Soc 428(4):3618-3629. https://doi.org/10.1093/mnras/sts295. arXiv:1210.8153 [astroph.HE]

Côté B, Fryer CL, Belczynski K, Korobkin O, Chruślińska M, Vassh N, Mumpower MR, Lippuner J, Sprouse TM, Surman R, Wollaeger R (2018) The origin of r-process elements in the milky way. Astrophys J 855(2):99. https://doi.org/10.3847/1538-4357/aaad67. arXiv:1710.05875 [astro-ph.GA]

Coward DM, Howell EJ, Piran T, Stratta G, Branchesi M, Bromberg O, Gendre B, Burman RR, Guetta D (2012) The Swift short gamma-ray burst rate density: implications for binary neutron star merger rates. Mon Not R Astron Soc 425(4):2668-2673. https://doi.org/10.1111/j.1365-2966.2012.21604.x. arXiv:1202.2179 [astro-ph.CO]

Croker KS, Zevin M, Farrah D, Nishimura KA, Tarlé G (2021) Cosmologically coupled compact objects: a single-parameter model for LIGO-Virgo mass and redshift distributions. Astrophys $\mathrm{J}$ Lett 921(2):L22. https://doi.org/10.3847/2041-8213/ac2fad. arXiv:2109.08146 [gr-qc]

Dall'Osso S, Piran T, Shaviv N (2014) Binary pulsar J0737-3039 - evidence for a new core collapse and neutron star formation mechanism. Mon Not R Astron Soc 438(2):1005-1013. https://doi.org/10. 1093/mnras/stt2188. arXiv:1308.0944 [astro-ph.HE]

de Freitas Pacheco JA, Regimbau T, Vincent S, Spallicci A (2006) Expected coalescence rates of Ns-Ns binaries for laser beam interferometers. Int J Mod Phys D 15(2):235-249. https://doi.org/10.1142/ S0218271806007699. arXiv:0510727 [astro-ph]

de Kool M (1990) Common envelope evolution and double cores of planetary nebulae. Astrophys J 358:189. https://doi.org/10.1086/168974

De Loore C, De Greve JP, de Cuyper JP (1975) Evolution of massive close binaries. II - The POST X-ray binary stage: Origin of run-away and binary pulsars. Astrophys Space Sci 36:219-225. https://doi. org/10.1007/BF00681952

De Luca V, Desjacques V, Franciolini G, Riotto A (2020a) The clustering evolution of primordial black holes. J Cosmol Astropart Phys 11:028. https://doi.org/10.1088/1475-7516/2020/11/028. arXiv: 2009.04731 [astro-ph.CO]

De Luca V, Franciolini G, Pani P, Riotto A (2020b) Primordial black holes confront LIGO/Virgo data: current situation. J Cosmol Astropart Phys 6:044. https://doi.org/10.1088/1475-7516/2020/06/044. arXiv:2005.05641 [astro-ph.CO]

de Mink SE, Belczynski K (2015) Merger rates of double neutron stars and stellar origin black holes: the impact of initial conditions on binary evolution predictions. Astrophys J 814:58. https://doi.org/10. 1088/0004-637X/814/1/58. arXiv:1506.03573 [astro-ph.HE]

de Mink SE, Mandel I (2016) The chemically homogeneous evolutionary channel for binary black hole mergers: rates and properties of gravitational-wave events detectable by advanced LIGO. Mon Not R Astron Soc 460:3545-3553. https://doi.org/10.1093/mnras/stw1219. arXiv:1603.02291 [astroph.HE]

Della Valle M, Guetta D, Cappellaro E, Amati L, Botticella MT, Branchesi M, Brocato E, Izzo L, PerezTorres MA, Stratta G (2018) GW170817: implications for the local kilonova rate and for surveys from ground-based facilities. Mon Not R Astron Soc 481(4):4355-4360. https://doi.org/10.1093/ mnras/sty2541. arXiv:1809.04295 [astro-ph.HE]

Dewi JDM, Podsiadlowski P, Sena A (2006) Double-core evolution and the formation of neutron star binaries with compact companions. Mon Not R Astron Soc 368:1742-1748. https://doi.org/10.1111/ j.1365-2966.2006.10233.x. arXiv:0602510 [astro-ph]

Di Carlo UN, Mapelli M, Giacobbo N, Spera M, Bouffanais Y, Rastello S, Santoliquido F, Pasquato M, Ballone A, Trani AA, Torniamenti S, Haardt F (2020) Binary black holes in young star clusters: the impact of metallicity. Mon Not R Astron Soc 498(1):495-506. https://doi.org/10.1093/mnras/ staa2286. arXiv:2004.09525 [astro-ph.HE]

Dichiara S, Troja E, O’Connor B, Marshall FE, Beniamini P, Cannizzo JK, Lien AY, Sakamoto T (2020) Short gamma-ray bursts within 200 Mpc. Mon Not R Astron Soc 492(4):5011-5022. https://doi.org/ 10.1093/mnras/staa124. arXiv:1912.08698 [astro-ph.HE] 
Doctor Z, Kessler R, Chen HY, Farr B, Finley DA, Foley RJ, Goldstein DA, Holz DE, Kim AG, Morganson E, Sako M et al (2017) A search for kilonovae in the dark energy survey. Astrophys J 837(1):57. https://doi.org/10.3847/1538-4357/aa5d09. arXiv:1611.08052 [astro-ph.HE]

Dominik M, Belczynski K, Fryer C, Holz DE, Berti E, Bulik T, Mandel I, O’Shaughnessy R (2012) Double compact objects. I. The Significance of the Common Envelope on Merger Rates. Astrophys J 759:52. https://doi.org/10.1088/0004-637X/759/1/52. arXiv:1202.4901 [astro-ph.HE]

Dominik M, Belczynski K, Fryer C, Holz DE, Berti E, Bulik T, Mandel I, O’Shaughnessy R (2013) Double compact objects. II. Cosmological merger rates. Astrophys J 779:72. https://doi.org/10.1088/ 0004-637X/779/1/72. arXiv:1308.1546 [astro-ph.HE]

Dominik M, Berti E, O’Shaughnessy R, Mandel I, Belczynski K, Fryer C, Holz DE, Bulik T, Pannarale F (2015) Double compact objects III: gravitational-wave detection rates. Astrophys J 806:263. https:// doi.org/10.1088/0004-637X/806/2/263. arXiv:1405.7016 [astro-ph.HE]

Dosopoulou F, Kalogera V (2016) Orbital evolution of mass-transferring eccentric binary systems. II. Secular evolution. Astrophys J 825(1):71. https://doi.org/10.3847/0004-637X/825/1/71. arXiv:1603. 06593 [astro-ph.SR]

Downing JMB, Benacquista MJ, Giersz M, Spurzem R (2011) Compact binaries in star clusters - II. Escapers and detection rates. Mon Not R Astron Soc 416:133-147. https://doi.org/10.1111/j.13652966.2011.19023.x. arXiv:1008.5060

Drozda P, Belczynski K, O’Shaughnessy R, Bulik T, Fryer CL (2020) Black hole - neutron star mergers: the first mass gap and kilonovae. arXiv e-prints arXiv:2009.06655 [astro-ph.HE]

du Buisson L, Marchant P, Podsiadlowski P, Kobayashi C, Abdalla FB, Taylor P, Mandel I, de Mink SE, Moriya TJ, Langer N (2020) Cosmic rates of black hole mergers and pair-instability supernovae from chemically homogeneous binary evolution. Mon Not R Astron Soc 499(4):5941-5959. https:// doi.org/10.1093/mnras/staa3225. arXiv:2002.11630 [astro-ph.HE]

Dvorkin I, Vangioni E, Silk J, Uzan JP, Olive KA (2016) Metallicity-constrained merger rates of binary black holes and the stochastic gravitational wave background. Mon Not $\mathrm{R}$ Astron Soc 461(4):3877-3885. https://doi.org/10.1093/mnras/stw1477. arXiv:1604.04288 [astro-ph.HE]

Eddington AS (1925) Circulating currents in rotating stars. Observatory 48:73-75

Eldridge JJ, Stanway ER (2016) BPASS predictions for binary black hole mergers. Mon Not R Astron Soc 462:3302-3313. https://doi.org/10.1093/mnras/stw1772. arXiv:1602.03790 [astro-ph.HE]

Eldridge JJ, Stanway ER, Tang PN (2019) A consistent estimate for gravitational wave and electromagnetic transient rates. Mon Not R Astron Soc 482(1):870-880. https://doi.org/10.1093/ mnras/sty2714. arXiv:1807.07659 [astro-ph.HE]

Endal AS, Sofia S (1978) The evolution of rotating stars. II - Calculations with time-dependent redistribution of angular momentum for 7- and 10-solar-mass stars. Astrophys J 220:279-290. https://doi.org/10.1086/155904

Farmer R, Renzo M, de Mink SE, Fishbach M, Justham S (2020) Constraints from gravitational-wave detections of binary black hole mergers on the ${ }^{12} \mathrm{C}(\alpha, \gamma)^{16} \mathrm{O}$ rate. Astrophys J Lett 902(2):L36. https://doi.org/10.3847/2041-8213/abbadd. arXiv:2006.06678 [astro-ph.HE]

Farr WM, Stevenson S, Miller MC, Mandel I, Farr B, Vecchio A (2017) Distinguishing spin-aligned and isotropic black hole populations with gravitational waves. Nature 548:426-429. https://doi.org/10. 1038/nature23453. arXiv:1706.01385 [astro-ph.HE]

Farrow N, Zhu XJ, Thrane E (2019) The mass distribution of galactic double neutron stars. Astrophys J 876(1):18. https://doi.org/10.3847/1538-4357/ab12e3. arXiv:1902.03300 [astro-ph.HE]

Fernández R, Quataert E, Kashiyama K, Coughlin ER (2018) Mass ejection in failed supernovae: variation with stellar progenitor. Mon Not R Astron Soc 476(2):2366-2383. https://doi.org/10.1093/ mnras/sty306. arXiv:1710.01735 [astro-ph.HE]

Finke JD, Razzaque S (2017) The binary black hole merger rate from ultraluminous X-ray source progenitors. Mon Not R Astron Soc 472(3):3683-3691. https://doi.org/10.1093/mnras/stx2248. arXiv:1708.09001 [astro-ph.HE]

Fishbach M, Holz DE (2017) Where are LIGOs big black holes? Astrophys J Lett 851:L25. https://doi. org/10.3847/2041-8213/aa9bf6. arXiv:1709.08584 [astro-ph.HE]

Fishbach M, Doctor Z, Callister T, Edelman B, Ye J, Essick R, Farr WM, Farr B, Holz DE (2021) When are LIGO/Virgos big black hole mergers? Astrophys J 912(2):98. https://doi.org/10.3847/15384357/abee11. arXiv:2101.07699 [astro-ph.HE]

Flannery BP, van den Heuvel EPJ (1975) On the origin of the binary pulsar PSR $1913+16$. Astron Astrophys 39:61-67 
Fong W, Berger E, Margutti R, Zauderer BA, Troja E, Czekala I, Chornock R, Gehrels N, Sakamoto T, Fox DB, Podsiadlowski P (2012) A Jet break in the X-ray light curve of short GRB 111020A: implications for energetics and rates. Astrophys J 756:189. https://doi.org/10.1088/0004-637X/756/ 2/189. arXiv:1204.5475 [astro-ph.HE]

Fong W, Berger E, Margutti R, Zauderer BA (2015) A decade of short-duration gamma-ray burst broadband afterglows: energetics, circumburst densities, and jet opening angles. Astrophys J 815:102. https://doi.org/10.1088/0004-637X/815/2/102. arXiv:1509.02922 [astro-ph.HE]

Ford KES, McKernan B (2021) Binary black hole merger rates in AGN disks versus nuclear star clusters: loud beats quiet. arXiv e-prints arXiv:2109.03212 [astro-ph.HE]

Foucart F, Hinderer T, Nissanke S (2018) Remnant baryon mass in neutron star-black hole mergers: predictions for binary neutron star mimickers and rapidly spinning black holes. Phys Rev D 98(8):081501. https://doi.org/10.1103/PhysRevD.98.081501. arXiv:1807.00011 [astro-ph.HE]

Fragione G, Banerjee S (2020) Demographics of neutron stars in young massive and open clusters. Astrophys J Lett 901(1):L16. https://doi.org/10.3847/2041-8213/abb671. arXiv:2006.06702 [astroph.GA]

Fragione G, Kocsis B (2018) Black hole mergers from an evolving population of globular clusters. Phys Rev Lett 121(16):161103. https://doi.org/10.1103/PhysRevLett.121.161103. arXiv:1806.02351 [astro-ph.GA]

Fragione G, Loeb A (2019) Black hole-neutron star mergers from triples. Mon Not R Astron Soc 486(3):4443-4450. https://doi.org/10.1093/mnras/stz1131. arXiv:1903.10511 [astro-ph.GA]

Fragione G, Loeb A (2019) Black hole-neutron star mergers from triples - II. The role of metallicity and spin-orbit misalignment. Mon Not R Astron Soc 490(4):4991-5001. https://doi.org/10.1093/mnras/ stz2902. arXiv:1907.08614 [astro-ph.GA]

Fragione G, Loeb A, Rasio FA (2020) Merging black holes in the low-mass and high-mass gaps from $2+$ 2 quadruple systems. Astrophys J Lett 895(1):L15. https://doi.org/10.3847/2041-8213/ab9093. arXiv:2002.11278 [astro-ph.GA]

Fryer CL, Belczynski K, Wiktorowicz G, Dominik M, Kalogera V, Holz DE (2012) Compact remnant mass function: dependence on the explosion mechanism and metallicity. Astrophys J 749:91. https:// doi.org/10.1088/0004-637X/749/1/91. arXiv:1110.1726 [astro-ph.SR]

Fujii MS, Tanikawa A, Makino J (2017) The detection rates of merging binary black holes originating from star clusters and their mass function. Publ Astron Soc Japan 69(6):94. https://doi.org/10.1093/ pasj/psx108. arXiv:1709.02058 [astro-ph.HE]

Gair JR, Mandel I, Sesana A, Vecchio A (2009) Probing seed black holes using future gravitational-wave detectors. Class Quantum Grav 26(20):204009. https://doi.org/10.1088/0264-9381/26/20/204009. arXiv:0907.3292

Galaudage S, Talbot C, Nagar T, Jain D, Thrane E, Mandel I (2021) Building better spin models for merging binary black holes: evidence for nonspinning and rapidly spinning nearly aligned subpopulations. Astrophys J Lett 921(1):L15. https://doi.org/10.3847/2041-8213/ac2f3c. arXiv: 2109.02424 [gr-qc]

Ge H, Webbink RF, Chen X, Han Z (2015) Adiabatic mass loss in binary stars. II. From zero-age main sequence to the base of the Giant Branch. Astrophys J 812(1):40. https://doi.org/10.1088/0004637X/812/1/40. arXiv:1507.04843 [astro-ph.SR]

Gerosa D (2017) Dgerosa/Gwdet: V0.1. https://doi.org/10.5281/zenodo.889966

Gerosa D, Fishbach M (2021) Hierarchical mergers of stellar-mass black holes and their gravitationalwave signatures. Nature Astron 5:749-760. https://doi.org/10.1038/s41550-021-01398-w. arXiv: 2105.03439 [astro-ph.HE]

Gessner A, Janka HT (2018) Hydrodynamical neutron-star kicks in electron-capture supernovae and implications for the CRAB supernova. Astrophys J 865(1):61. https://doi.org/10.3847/1538-4357/ aadbae. arXiv:1802.05274 [astro-ph.HE]

Ghodla S, van Zeist WGJ, Eldridge JJ, Stevance HF, Stanway ER (2021) Forward modelling the LIGO/ VIRGO O3a GW transient mass distributions with BPASS. arXiv e-prints arXiv:2105.05783 [astroph.HE]

Giacobbo N, Mapelli M (2018) The progenitors of compact-object binaries: impact of metallicity, common envelope and natal kicks. Mon Not R Astron Soc 480(2):2011-2030. https://doi.org/10. 1093/mnras/sty1999. arXiv:1806.00001 [astro-ph.HE]

Giacobbo N, Mapelli M (2020) Revising natal kick prescriptions in population synthesis simulations. Astrophys J 891(2):141. https://doi.org/10.3847/1538-4357/ab7335. arXiv:1909.06385 [astroph.HE] 
Ginat YB, Perets HB (2021) Analytical, statistical approximate solution of dissipative and nondissipative binary-single stellar encounters. Phys Rev X 11(3):031020. https://doi.org/10.1103/PhysRevX.11. 031020. arXiv:2011.00010 [astro-ph.GA]

Gompertz BP, Levan AJ, Tanvir NR (2020) A search for neutron star-black hole binary mergers in the short gamma-ray burst population. Astrophys J 895(1):58. https://doi.org/10.3847/1538-4357/ ab8d24. arXiv:2001.08706 [astro-ph.HE]

Graff PB, Buonanno A, Sathyaprakash BS (2015) Missing link: Bayesian detection and measurement of intermediate-mass black-hole binaries. Phys Rev D 92(2):022002. https://doi.org/10.1103/ PhysRevD.92.022002. arXiv:1504.04766 [gr-qc]

Greene JE, Strader J, Ho LC (2020) Intermediate-mass black holes. Annu Rev Astron Astrophys 58:257-312. https://doi.org/10.1146/annurev-astro-032620-021835. arXiv:1911.09678 [astroph.GA]

Gröbner M, Ishibashi W, Tiwari S, Haney M, Jetzer P (2020) Binary black hole mergers in AGN accretion discs: gravitational wave rate density estimates. Astron Astrophys 638:A119. https://doi. org/10.1051/0004-6361/202037681. arXiv:2005.03571 [astro-ph.GA]

Grunthal K, Kramer M, Desvignes G (2021) Revisiting the Galactic Double Neutron Star merger and LIGO detection rates. Mon Not R Astron Soc https://doi.org/10.1093/mnras/stab2198. arXiv:2107. 13307 [astro-ph.HE]

Hamers AS, Thompson TA (2019) Double neutron star mergers from hierarchical triple-star systems. Astrophys J 883(1):23. https://doi.org/10.3847/1538-4357/ab3b06. arXiv:1907.08297 [astro-ph.HE]

Hamers AS, Bar-Or B, Petrovich C, Antonini F (2018) The impact of vector resonant relaxation on the evolution of binaries near a massive black hole: implications for gravitational-wave sources. Astrophys J 865(1):2. https://doi.org/10.3847/1538-4357/aadae2. arXiv:1805.10313 [astro-ph.HE]

Hamers AS, Fragione G, Neunteufel P, Kocsis B (2021) First and second-generation black hole and neutron star mergers in 2+2 quadruples: population statistics. Mon Not R Astron Soc https://oi.org/ 10.1093/mnras/stab2136. arXiv:2103.03782 [astro-ph.HE]

Han ZW, Ge HW, Chen XF, Chen HL (2020) Binary population synthesis. Res Astron Astrophys 20(10):161. https://doi.org/10.1088/1674-4527/20/10/161. arXiv:2009.08611 [astro-ph.SR]

Hannam M, Brown DA, Fairhurst S, Fryer CL, Harry IW (2013) When can gravitational-wave observations distinguish between black holes and neutron stars? Astrophys J Lett 766:L14. https:// doi.org/10.1088/2041-8205/766/1/L14. arXiv:1301.5616 [gr-qc]

Hartwig T, Volonteri M, Bromm V, Klessen RS, Barausse E, Magg M, Stacy A (2016) Gravitational waves from the remnants of the first stars. Mon Not R Astron Soc 460(1):L74-L78. https://doi.org/ 10.1093/mnrasl/slw074. arXiv:1603.05655 [astro-ph.GA]

Haster CJ, Antonini F, Kalogera V, Mandel I (2016) N-Body dynamics of intermediate mass-ratio inspirals in star clusters. Astrophys J 832:192. https://doi.org/10.3847/0004-637X/832/2/192. arXiv: 1606.07097 [astro-ph.HE]

Haster CJ, Wang Z, Berry CPL, Stevenson S, Veitch J, Mandel I (2016) Inference on gravitational waves from coalescences of stellar-mass compact objects and intermediate-mass black holes. Mon Not R Astron Soc 457:4499-4506. https://doi.org/10.1093/mnras/stw233. arXiv:1511.01431 [astro-ph.HE]

Heger A, Langer N, Woosley SE (2000) Presupernova evolution of rotating massive stars. I. Numerical Method and Evolution of the Internal Stellar Structure. Astrophys J 528:368-396. https://doi.org/10. 1086/308158. arXiv:9904132 [astro-ph]

Heggie D, Hut P (2003) The gravitational million-body problem: a multidisciplinary approach to star cluster dynamics. Cambridge University Press, Cambridge

Hijikawa K, Tanikawa A, Kinugawa T, Yoshida T, Umeda H (2021) On the population III binary black hole mergers beyond the pair-instability mass gap. Mon Not R Astron Soc https://doi.org/10.1093/ mnrasl/slab052. arXiv:2104.13384 [astro-ph.HE]

Hirai R, Sato T, Podsiadlowski P, Vigna-Gómez A, Mandel I (2020) Formation pathway for lonely stripped-envelope supernova progenitors: implications for Cassiopeia A. Mon Not R Astron Soc 499(1):1154-1171. https://doi.org/10.1093/mnras/staa2898. arXiv:2008.05076 [astro-ph.SR]

Hirano S, Hosokawa T, Yoshida N, Umeda H, Omukai K, Chiaki G, Yorke HW (2014) One hundred first stars: protostellar evolution and the final masses. Astrophys J 781(2):60. https://doi.org/10.1088/ 0004-637X/781/2/60. arXiv:1308.4456 [astro-ph.CO]

Hoang BM, Naoz S, Kocsis B, Rasio FA, Dosopoulou F (2018) Black hole mergers in galactic nuclei induced by the eccentric Kozai-Lidov effect. Astrophys J 856:140. https://doi.org/10.3847/15384357/aaafce. arXiv:1706.09896 [astro-ph.HE] 
Hoang BM, Naoz S, Kremer K (2020) Neutron star-black hole mergers from gravitational-wave captures. Astrophys J 903(1):8. https://doi.org/10.3847/1538-4357/abb66a. arXiv:2007.08531 [astro-ph.HE]

Hobbs G, Lorimer DR, Lyne AG, Kramer M (2005) A statistical study of 233 pulsar proper motions. Mon Not R Astron Soc 360:974-992. https://doi.org/10.1111/j.1365-2966.2005.09087.x. arXiv:0504584 [astro-ph]

Hong J, Vesperini E, Askar A, Giersz M, Szkudlarek M, Bulik T (2018) Binary black hole mergers from globular clusters: the impact of globular cluster properties. Mon Not $\mathrm{R}$ Astron Soc 480(4):5645-5656. https://doi.org/10.1093/mnras/sty2211. arXiv:1808.04514 [astro-ph.HE]

Hotokezaka K, Piran T, Paul M (2015) Short-lived ${ }^{244} \mathrm{Pu}$ points to compact binary mergers as sites for heavy r-process nucleosynthesis. Nature Phys 11:1042. https://doi.org/10.1038/nphys3574. arXiv: 1510.00711 [astro-ph.HE]

Hulse RA, Taylor JH (1975) Discovery of a pulsar in a binary system. Astrophys J Lett 195:L51-L53. https://doi.org/10.1086/181708

Hut P (1981) Tidal evolution in close binary systems. Astron Astrophys 99:126-140

Inayoshi K, Kashiyama K, Visbal E, Haiman Z (2016) Gravitational wave background from Population III binary black holes consistent with cosmic reionization. Mon Not $\mathrm{R}$ Astron Soc 461(3):2722-2727. https://doi.org/10.1093/mnras/stw1431. arXiv:1603.06921 [astro-ph.GA]

Inayoshi K, Hirai R, Kinugawa T, Hotokezaka K (2017) Formation pathway of Population III coalescing binary black holes through stable mass transfer. Mon Not R Astron Soc 468(4):5020-5032. https:// doi.org/10.1093/mnras/stx757. arXiv:1701.04823 [astro-ph.HE]

Inoue Y, Tanaka YT, Isobe N (2016) Binary black hole merger rates inferred from luminosity function of ultra-luminous X-ray sources. Mon Not R Astron Soc 461(4):4329-4334. https://doi.org/10.1093/ mnras/stw1637. arXiv:1602.05554 [astro-ph.HE]

Ivanova N, Justham S, Chen X, De Marco O, Fryer CL, Gaburov E, Ge H, Glebbeek E, Han Z, Li XD, Lu G, Marsh T, Podsiadlowski P, Potter A, Soker N, Taam R, Tauris TM, van den Heuvel EPJ, Webbink RF (2013) Common envelope evolution: where we stand and how we can move forward. Astron Astrophys Rev 21:59. https://doi.org/10.1007/s00159-013-0059-2. arXiv:1209.4302 [astroph.HE]

Jin ZP, Fan YZ, Wei DM (2016) An r-process macronova/kilonova in GRB 060614: evidence for the merger of a neutron star-black hole binary. EPJ Web Conf 109:08002. https://doi.org/10.1051/ epjconf/201610908002. arXiv:1512.04192 [astro-ph.HE]

Jin ZP, Li X, Wang H, Wang YZ, He HN, Yuan Q, Zhang FW, Zou YC, Fan YZ, Wei DM (2018) Short GRBs: opening angles, local neutron star merger rate, and off-axis events for GRB/GW association. Astrophys J 857(2):128. https://doi.org/10.3847/1538-4357/aab76d. arXiv:1708.07008 [astroph.HE]

Kalogera V, Kim C, Lorimer DR, Burgay M, D’Amico N, Possenti A, Manchester RN, Lyne AG, Joshi BC, McLaughlin MA, Kramer M, Sarkissian JM, Camilo F (2004) The cosmic coalescence rates for double neutron star binaries. Astrophys J 601:L179-L182. Erratum-ibid. 614 (2004) L137

Kalogera V, Belczynski K, Kim C, O’Shaughnessy R, Willems B (2007) Formation of double compact objects. Phys Rep 442:75-108. https://doi.org/10.1016/j.physrep.2007.02.008. arXiv:0612144 [astro-ph]

Kalogera V, Berry CP, Colpi M, Fairhurst S, Justham S, Mandel I, Mangiagli A, Mapelli M, Mills C, Sathyaprakash BS, Schneider R, Tauris T, Valiante R (2019) Deeper, wider, sharper: nextgeneration ground-based gravitational-wave observations of binary black holes. arXiv e-prints arXiv:1903.09220 [astro-ph.HE]

Kasen D, Badnell NR, Barnes J (2013) Opacities and spectra of the r-process ejecta from neutron star mergers. Astrophys J 774(1):25. https://doi.org/10.1088/0004-637X/774/1/25. arXiv:1303.5788 [astro-ph.HE]

Kasen D, Metzger B, Barnes J, Quataert E, Ramirez-Ruiz E (2017) Origin of the heavy elements in binary neutron-star mergers from a gravitational-wave event. Nature 551(7678):80-84. https://doi.org/10. 1038/nature24453. arXiv:1710.05463 [astro-ph.HE]

Kasliwal MM, Nakar E, Singer LP, Kaplan DL, Cook DO, Van Sistine A, Lau RM, Fremling C, Gottlieb O et al (2017) Illuminating gravitational waves: a concordant picture of photons from a neutron star merger. Science 358(6370):1559-1565. https://doi.org/10.1126/science.aap9455. arXiv:1710.05436 [astro-ph.HE]

Kasliwal MM, Anand S, Ahumada T, Stein R, Carracedo AS, Andreoni I, Coughlin MW, Singer LP et al (2020) Kilonova luminosity function constraints based on Zwicky transient facility searches for 13 
neutron star merger triggers during O3. Astrophys J 905(2):145. https://doi.org/10.3847/1538-4357/ abc335. arXiv:2006.11306 [astro-ph.HE]

Kim C, Kalogera V, Lorimer DR (2003) The probability distribution of binary pulsar coalescence rates. I. Double neutron star systems in the galactic field. Astrophys J 584:985-995. https://doi.org/10. 1086/345740. arXiv:0207408 [astro-ph]

Kim C, Kalogera V, Lorimer DR (2006) Effect of PSR J0737-3039 on the DNS merger rate and implications for GW detection. In: A life with stars. arXiv e-prints arXiv:astro-ph/0608280

Kim C, Perera BBP, McLaughlin MA (2015) Implications of PSR J0737-3039B for the Galactic NS-NS binary merger rate. Mon Not R Astron Soc 448(1):928-938. https://doi.org/10.1093/mnras/stu2729. arXiv:1308.4676 [astro-ph.SR]

Kinugawa T, Inayoshi K, Hotokezaka K, Nakauchi D, Nakamura T (2014) Possible indirect confirmation of the existence of Pop III massive stars by gravitational wave. Mon Not R Astron Soc 442:2963-2992. https://doi.org/10.1093/mnras/stu1022. arXiv:1402.6672 [astro-ph.HE]

Kinugawa T, Nakamura T, Nakano H (2021) Formation of binary black holes similar to GW190521 with a total mass of $150 \mathrm{M}$ solar masses from population III binary star evolution. Mon Not R Astron Soc 501(1):L49-L53. https://doi.org/10.1093/mnrasl/slaa191. arXiv:2009.06922 [astro-ph.HE]

Kippenhahn R, Meyer-Hofmeister E (1977) On the radii of accreting main sequence stars. Astron Astrophys 54:539-542

Klencki J, Moe M, Gladysz W, Chruslinska M, Holz DE, Belczynski K (2018) Impact of inter-correlated initial binary parameters on double black hole and neutron star mergers. Astron Astrophys 619:A77. https://doi.org/10.1051/0004-6361/201833025. arXiv:1808.07889 [astro-ph.HE]

Klencki J, Nelemans G, Istrate AG, Chruslinska M (2021) It has to be cool: Supergiant progenitors of binary black hole mergers from common-envelope evolution. Astron Astrophys 645:A54. https:// doi.org/10.1051/0004-6361/202038707. arXiv:2006.11286 [astro-ph.SR]

Kobayashi C, Karakas AI, Lugaro M (2020) The origin of elements from carbon to uranium. Astrophys J 900(2):179. https://doi.org/10.3847/1538-4357/abae65. arXiv:2008.04660 [astro-ph.GA]

Kopparapu RK, Hanna CR, Kalogera V, O’Shaughnessy R, Gonzalez G, Brady PR, Fairhurst S (2008) Host galaxies catalog used in LIGO searches for compact binary coalescence events. Astrophys J 675(2):1459-1467. https://doi.org/10.1086/527348

Korol V, Mandel I, Miller MC, Church RP, Davies MB (2020) Merger rates in primordial black hole clusters without initial binaries. Mon Not R Astron Soc 496(1):994-1000. https://doi.org/10.1093/ mnras/staa1644. arXiv:1911.03483 [astro-ph.HE]

Kozai Y (1962) Secular perturbations of asteroids with high inclination and eccentricity. Astron J 67:591. https://doi.org/10.1086/108790

Kremer K, Ye CS, Rui NZ, Weatherford NC, Chatterjee S, Fragione G, Rodriguez CL, Spera M, Rasio FA (2020) Modeling dense star clusters in the milky way and beyond with the CMC cluster catalog. Astrophys J Suppl 247(2):48. https://doi.org/10.3847/1538-4365/ab7919. arXiv:1911.00018 [astroph.HE]

Kroupa P (2002) The initial mass function of stars: evidence for uniformity in variable systems. Science 295:82-91. https://doi.org/10.1126/science.1067524. arXiv:0201098 [astro-ph]

Kroupa P, Subr L, Jerabkova T, Wang L (2020) Very high redshift quasars and the rapid emergence of supermassive black holes. Mon Not R Astron Soc 498(4):5652-5683. https://doi.org/10.1093/mnras/ staa2276. arXiv:2007.14402 [astro-ph.GA]

Kruckow MU, Tauris TM, Langer N, Kramer M, Izzard RG (2018) Progenitors of gravitational wave mergers: binary evolution with the stellar grid-based code COMBINE. Mon Not R Astron Soc 481:1908-1949. https://doi.org/10.1093/mnras/sty2190. arXiv:1801.05433 [astro-ph.SR]

Kulkarni SR, Hut P, McMillan S (1993) Stellar black holes in globular clusters. Nature 364:421-423. https://doi.org/10.1038/364421a0

Kumamoto J, Fujii MS, Tanikawa A (2020) Merger rate density of binary black holes formed in open clusters. Mon Not R Astron Soc 495(4):4268-4278. https://doi.org/10.1093/mnras/staa1440. arXiv: 2001.10690 [astro-ph.HE]

Lamb GP, Lyman JD, Levan AJ, Tanvir NR, Kangas T, Fruchter AS, Gompertz B, Hjorth J, Mandel I, Oates SR, Steeghs D, Wiersema K (2019) The optical afterglow of GW170817 at one year postmerger. Astrophys J Lett 870:L15. https://doi.org/10.3847/2041-8213/aaf96b. arXiv:1811.11491 [astro-ph.HE]

Lamberts A, Garrison-Kimmel S, Clausen DR, Hopkins PF (2016) When and where did GW150914 form? Mon Not R Astron Soc 463:L31-L35. https://doi.org/10.1093/mnrasl/slw152. arXiv:1605. 08783 [astro-ph.HE] 
Laplace E, Götberg Y, de Mink SE, Justham S, Farmer R (2020) The expansion of stripped-envelope stars: consequences for supernovae and gravitational-wave progenitors. Astron Astrophys 637:A6. https://doi.org/10.1051/0004-6361/201937300. arXiv:2003.01120 [astro-ph.SR]

Lau MYM, Hirai R, González-Bolívar M, Price DJ, De Marco O, Mandel I (2021) Common envelopes in massive stars: the role of radiation pressure and recombination energy in ejecting red supergiant envelopes. arXiv e-prints arXiv:2111.00923 [astro-ph.SR]

Law-Smith JAP, Everson RW, Ramirez-Ruiz E, de Mink SE, van Son LAC, Götberg Y, Zellmann S, Vigna-Gómez A, Renzo M, Wu S, Schrøder SL, Foley RJ, Hutchinson-Smith T (2020) Successful common envelope ejection and binary neutron star formation in 3D hydrodynamics. arXiv e-prints arXiv:2011.06630 [astro-ph.HE]

Laycock SGT, Maccarone TJ, Christodoulou DM (2015) Revisiting the dynamical case for a massive black hole in IC10 X-1. Mon Not R Astron Soc 452:L31-L35. https://doi.org/10.1093/mnrasl/ slv082. arXiv:1506.03882 [astro-ph.HE]

Lee WH, Ramirez-Ruiz E, van de Ven G (2010) Short gamma-ray bursts from dynamically assembled compact binaries in globular clusters: pathways, rates, hydrodynamics, and cosmological setting. Astrophys J 720(1):953-975. https://doi.org/10.1088/0004-637X/720/1/953. arXiv:0909.2884 [astro-ph.HE]

Li LX, Paczyński B (1998) Transient events from neutron star mergers. Astrophys J Lett 507(1):L59L62. https://doi.org/10.1086/311680. arXiv:9807272 [astro-ph]

Li X, Hu YM, Jin ZP, Fan YZ, Wei DM (2017) Neutron star-black hole coalescence rate inferred from macronova observations. Astrophys J Lett 844(2):L22. https://doi.org/10.3847/2041-8213/aa7fb2. arXiv:1611.01760 [astro-ph.HE]

Lidov ML (1962) The evolution of orbits of artificial satellites of planets under the action of gravitational perturbations of external bodies. Planet Space Sci 9:719-759. https://doi.org/10.1016/00320633(62)90129-0

Lipunov VM, Pruzhinskaya MV (2014) Scenario Machine: fast radio bursts, short gamma-ray burst, dark energy and Laser Interferometer Gravitational-wave Observatory silence. Mon Not R Astron Soc 440(2):1193-1199. https://doi.org/10.1093/mnras/stu313. arXiv:1312.3143 [astro-ph.HE]

Lipunov VM, Postnov KA, Prokhorov ME (1997) Formation and coalescence of relativistic binary stars: the effect of kick velocity. Mon Not R Astron Soc 288:245-259 arXiv:9702060 [astro-ph]

Lipunov VM, Kornilov V, Gorbovskoy E, Tiurina N, Balanutsa P, Kuznetsov A (2017) The first gravitational-wave burst GW150914, as predicted by the scenario machine. New Astron 51:122-127. https://doi.org/10.1016/j.newast.2016.08.017. arXiv:1605.01604 [astro-ph.HE]

Liu B, Bromm V (2021) Gravitational waves from the remnants of the first stars in nuclear star clusters. Mon Not R Astron Soc 506(4):5451-5467. https://doi.org/10.1093/mnras/stab2028. arXiv:2106. 02244 [astro-ph.GA]

Liu B, Lai D (2018) Black hole and neutron star binary mergers in triple systems: merger fraction and spin-orbit misalignment. Astrophys J 863(1):68. https://doi.org/10.3847/1538-4357/aad09f. arXiv: 1805.03202 [astro-ph.HE]

Lovegrove E, Woosley SE (2013) Very low energy supernovae from neutrino mass loss. Astrophys J 769(2):109. https://doi.org/10.1088/0004-637X/769/2/109. arXiv:1303.5055 [astro-ph.HE]

Lu W, Beniamini P, Bonnerot C (2021) On the formation of GW190814. Mon Not R Astron Soc 500(2):1817-1832. https://doi.org/10.1093/mnras/staa3372. arXiv:2009.10082 [astro-ph.HE]

Lyman JD, Lamb GP, Levan AJ, Mandel I, Tanvir NR, Kobayashi S, Gompertz B, Hjorth J et al (2018) The optical afterglow of the short gamma-ray burst associated with GW170817. Nat Astron 2:751-754. https://doi.org/10.1038/s41550-018-0511-3. arXiv:1801.02669 [astro-ph.HE]

Madau P, Dickinson M (2014) Cosmic star-formation history. Annu Rev Astron Astrophys 52:415-486. https://doi.org/10.1146/annurev-astro-081811-125615. arXiv:1403.0007

Maeder A, Meynet G (2000) The evolution of rotating stars. Annu Rev Astron Astrophys 38:143-190. https://doi.org/10.1146/annurev.astro.38.1.143. arXiv:0004204 [astro-ph]

Mandel I (2016) Estimates of black hole natal kick velocities from observations of low-mass X-ray binaries. Mon Not R Astron Soc 456:578-581. https://doi.org/10.1093/mnras/stv2733. arXiv:1510. 03871 [astro-ph.HE]

Mandel I, de Mink SE (2016) Merging binary black holes formed through chemically homogeneous evolution in short-period stellar binaries. Mon Not R Astron Soc 458:2634-2647. https://doi.org/10. 1093/mnras/stw379. arXiv:1601.00007 [astro-ph.HE]

Mandel I, Farmer A (2018) Merging stellar-mass binary black holes. ArXiv e-prints arXiv:1806.05820 [astro-ph.HE] 
Mandel I, Müller B (2020) Simple recipes for compact remnant masses and natal kicks. Mon Not R Astron Soc 499(3):3214-3221. https://doi.org/10.1093/mnras/staa3043. arXiv:2006.08360 [astroph.HE]

Mandel I, O'Shaughnessy R (2010) Compact binary coalescences in the band of ground-based gravitational-wave detectors. Class Quantum Grav 27(11):114007. https://doi.org/10.1088/02649381/27/11/114007. arXiv:0912.1074

Mandel I, Brown DA, Gair JR, Miller MC (2008) Rates and characteristics of intermediate mass ratio inspirals detectable by advanced LIGO. Astrophys J 681:1431-1447. https://doi.org/10.1086/ 588246. arXiv:0705.0285

Mandhai S, Tanvir N, Lamb G, Levan A, Tsang D (2018) The rate of short-duration gamma-ray bursts in the local universe. Galaxies 6(4):130. https://doi.org/10.3390/galaxies6040130. arXiv:1812.00507 [astro-ph.HE]

Mapelli M (2016) Massive black hole binaries from runaway collisions: the impact of metallicity. Mon Not R Astron Soc 459:3432-3446. https://doi.org/10.1093/mnras/stw869. arXiv:1604.03559

Mapelli M (2021) Formation channels of single and binary stellar-mass black holes. In: Bambi C, Katsanevas S, Kokkotas KD (eds) Handbook of gravitational wave astronomy. Springer, Singapore. https://doi.org/10.1007/978-981-15-4702-7_16-1. arXiv:2106.00699 [astro-ph.HE]

Mapelli M, Giacobbo N (2018) The cosmic merger rate of neutron stars and black holes. Mon Not R Astron Soc 479(4):4391-4398. https://doi.org/10.1093/mnras/sty1613. arXiv:1806.04866 [astroph.HE]

Mapelli M, Giacobbo N, Ripamonti E, Spera M (2017) The cosmic merger rate of stellar black hole binaries from the Illustris simulation. Mon Not R Astron Soc 472(2):2422-2435. https://doi.org/10. 1093/mnras/stx2123. arXiv:1708.05722 [astro-ph.GA]

Mapelli M, Santoliquido F, Bouffanais Y, Arca Sedda MA, Artale MC, Ballone A (2021) Mass and rate of hierarchical black hole mergers in young, globular and nuclear star clusters. Symmetry 13(9):1678. https://doi.org/10.3390/sym13091678. arXiv:2007.15022 [astro-ph.HE]

Marchant P, Langer N, Podsiadlowski P, Tauris TM, Moriya TJ (2016) A new route towards merging massive black holes. Astron Astrophys 588:A50. https://doi.org/10.1051/0004-6361/201628133. arXiv:1601.03718 [astro-ph.SR]

Marchant P, Langer N, Podsiadlowski P, Tauris TM, de Mink S, Mandel I, Moriya TJ (2017) Ultraluminous X-ray sources and neutron-star-black-hole mergers from very massive close binaries at low metallicity. Astron Astrophys 604:A55. https://doi.org/10.1051/0004-6361/201630188. arXiv: 1705.04734 [astro-ph.HE]

Margutti R, Chornock R (2021) First multimessenger observations of a neutron star merger. Annu Rev Astron Astrophys 59:155-202. https://doi.org/10.1146/annurev-astro-112420-030742. arXiv:2012. 04810 [astro-ph.HE]

Marigo P, Girardi L, Chiosi C, Wood PR (2001) Zero-metallicity stars. I. Evolution at constant mass. Astron Astrophys 371:152-173. https://doi.org/10.1051/0004-6361:20010309. arXiv:0102253 [astro-ph]

Martinez MAS, Fragione G, Kremer K, Chatterjee S, Rodriguez CL, Samsing J, Ye CS, Weatherford NC, Zevin M, Naoz S, Rasio FA (2020) Black hole mergers from hierarchical triples in dense star clusters. Astrophys J 903(1):67. https://doi.org/10.3847/1538-4357/abba25. arXiv:2009.08468 [astro-ph.GA]

McKernan B, Ford KES, Lyra W, Perets HB (2012) Intermediate mass black holes in AGN discs - I. Production and growth. Mon Not R Astron Soc 425:460-469. https://doi.org/10.1111/j.1365-2966. 2012.21486.x. arXiv:1206.2309

McKernan B, Ford KES, Bellovary J, Leigh NWC, Haiman Z, Kocsis B, Lyra W, Mac Low MM, Metzger B, O’Dowd M, Endlich S, Rosen DJ (2018) Constraining stellar-mass black hole mergers in AGN disks detectable with LIGO. Astrophys J 866(1):66. https://doi.org/10.3847/1538-4357/ aadae5. arXiv:1702.07818 [astro-ph.HE]

McKernan B, Ford KES, O’Shaughnessy R (2020) Black hole, neutron star, and white dwarf merger rates in AGN discs. Mon Not R Astron Soc 498(3):4088-4094. https://doi.org/10.1093/mnras/staa2681. arXiv:2002.00046 [astro-ph.HE]

Mennekens N, Vanbeveren D (2014) Massive double compact object mergers: gravitational wave sources and r-process element production sites. Astron Astrophys 564:A134. https://doi.org/10.1051/00046361/201322198. arXiv:1307.0959 [astro-ph.SR]

Metzger BD (2019) Kilonovae. Living Rev Relativ 23:1. https://doi.org/10.1007/s41114-019-0024-0. arXiv:1910.01617 [astro-ph.HE] 
Metzger BD, Martínez-Pinedo G, Darbha S, Quataert E, Arcones A, Kasen D, Thomas R, Nugent P, Panov IV, Zinner NT (2010) Electromagnetic counterparts of compact object mergers powered by the radioactive decay of r-process nuclei. Mon Not R Astron Soc 406:2650-2662. https://doi.org/10. 1111/j.1365-2966.2010.16864.x. arXiv:1001.5029 [astro-ph.HE]

Miller MC, Colbert EJM (2004) Intermediate-mass black holes. Int J Mod Phys D 13:1-64. https://doi. org/10.1142/S0218271804004426. arXiv:0308402 [astro-ph]

Miller MC, Lauburg VM (2009) Mergers of stellar-mass black holes in nuclear star clusters. Astrophys J 692:917-923. https://doi.org/10.1088/0004-637X/692/1/917. arXiv:0804.2783

Miller-Jones JCA, Bahramian A, Orosz JA, Mandel I, Gou L, Maccarone TJ, Neijssel CJ, Zhao X, Ziółkowski J, Reid MJ, Uttley P, Zheng X, Byun DY, Dodson R, Grinberg V, Jung T, Kim JS, Marcote B, Markoff S, Rioja MJ, Rushton AP, Russell DM, Sivakoff GR, Tetarenko AJ, Tudose V, Wilms J (2021) Cygnus X-1 contains a 21-solar mass black hole-Implications for massive star winds. Science 371(6533):1046-1049. https://doi.org/10.1126/science.abb3363. arXiv:2102.09091 [astro-ph.HE]

Mirabel F (2017) The formation of stellar black holes. New Astron Rev 78:1-15. https://doi.org/10.1016/ j.newar.2017.04.002

Mochkovitch R, Daigne F, Duque R, Zitouni H (2021) Prospects for kilonova signals in the gravitationalwave era. Astron Astrophys 651:A83. https://doi.org/10.1051/0004-6361/202140689. arXiv:2103. 00943 [astro-ph.HE]

Moe M, Di Stefano R (2017) Mind your Ps and Qs: the interrelation between period (P) and mass-ratio (Q) distributions of binary stars. Astrophys J Suppl 230:15. https://doi.org/10.3847/1538-4365/ aa6fb6. arXiv:1606.05347 [astro-ph.SR]

Mooley KP, Nakar E, Hotokezaka K, Hallinan G, Corsi A, Frail DA, Horesh A, Murphy T, Lenc E, Kaplan DL, De K, Dobie D, Chandra P, Deller A, Gottlieb O, Kasliwal MM, Kulkarni SR, Myers ST, Nissanke S, Piran T, Lynch C, Bhalerao V, Bourke S, Bannister KW, Singer LP (2018) A mildly relativistic wide-angle outflow in the neutron-star merger event GW170817. Nature 554(7691):207-210. https://doi.org/10.1038/nature25452. arXiv:1711.11573 [astro-ph.HE]

Morscher M, Pattabiraman B, Rodriguez C, Rasio FA, Umbreit S (2015) The dynamical evolution of stellar black holes in globular clusters. Astrophys J 800:9. https://doi.org/10.1088/0004-637X/800/1/ 9. arXiv:1409.0866

Mösta P, Radice D, Haas R, Schnetter E, Bernuzzi S (2020) A magnetar engine for short GRBs and kilonovae. Astrophys J Lett 901(2):L37. https://doi.org/10.3847/2041-8213/abb6ef. arXiv:2003. 06043 [astro-ph.HE]

Müller B (2020) Hydrodynamics of core-collapse supernovae and their progenitors. Living Rev Comput Astrophys 6:3. https://doi.org/10.1007/s41115-020-0008-5. arXiv:2006.05083 [astro-ph.SR]

Nadezhin DK (1980) Some secondary indications of gravitational collapse. Astrophys Space Sci 69(1):115-125. https://doi.org/10.1007/BF00638971

Nakar E (2007) Short-hard gamma-ray bursts. Phys Rep 442:166-236. https://doi.org/10.1016/j.physrep. 2007.02.005. arXiv:0701748 [astro-ph]

Nakar E, Gal-Yam A, Fox DB (2006) The local rate and the progenitor lifetimes of short-hard gamma-ray bursts: synthesis and predictions for LIGO. Astrophys J 650:281-290 arXiv:0511254 [astro-ph]

Naoz S (2016) The eccentric Kozai-Lidov effect and its applications. Annu Rev Astron Astrophys 54:441-489. https://doi.org/10.1146/annurev-astro-081915-023315. arXiv:1601.07175 [astroph.EP]

Narayan R, Piran T, Shemi A (1991) Neutron star and black hole binaries in the Galaxy. Astrophys J Lett 379:L17-L20. https://doi.org/10.1086/186143

Neijssel CJ, Vigna-Gómez A, Stevenson S, Barrett JW, Gaebel SM, Broekgaarden FS, de Mink SE, Szécsi D, Vinciguerra S, Mandel I (2019) The effect of the metallicity-specific star formation history on double compact object mergers. Mon Not R Astron Soc 490(3):3740-3759. https://doi.org/10. 1093/mnras/stz2840. arXiv:1906.08136 [astro-ph.SR]

Neijssel CJ, Vinciguerra S, Vigna-Gómez A, Hirai R, Miller-Jones JCA, Bahramian A, Maccarone TJ, Mandel I (2021) Wind mass-loss rates of stripped stars inferred from Cygnus X-1. Astrophys J 908(2):118. https://doi.org/10.3847/1538-4357/abde4a. arXiv:2102.09092 [astro-ph.SR]

Nelemans G, Yungelson LR, Portegies Zwart SF (2001) The gravitational wave signal from the Galactic disk population of binaries containing two compact objects. Astron Astrophys 375:890-898. https:// doi.org/10.1051/0004-6361:20010683. arXiv:0105221 [astro-ph] 
Ng KKY, Vitale S, Farr WM, Rodriguez CL (2021) Probing multiple populations of compact binaries with third-generation gravitational-wave detectors. Astrophys J Lett 913(1):L5. https://doi.org/10. 3847/2041-8213/abf8be. arXiv:2012.09876 [astro-ph.CO]

Nitz AH, Capano CD, Kumar S, Wang YF, Kastha S, Schäfer M, Dhurkunde R, Cabero M (2021a) 3-OGC: catalog of gravitational waves from compact-binary mergers. Astrophys J 922:76. https:// doi.org/10.3847/1538-4357/ac1c03. arXiv:2105.09151 [astro-ph.HE]

Nitz AH, Kumar S, Wang YF, Kastha S, Wu S, Schäfer M, Dhurkunde R, Capano CD (2021b) 4-OGC: catalogof gravitational waves from compact-binary mergers. arXiv eprints arXiv:2112.06878 [astroph.HE]

O'Leary RM, Rasio FA, Fregeau JM, Ivanova N, O'Shaughnessy R (2006) Binary mergers and growth of black holes in dense star clusters. Astrophys J 637(2):937-951. https://doi.org/10.1086/498446. arXiv:0508224 [astro-ph]

O'Leary RM, Kocsis B, Loeb A (2009) Gravitational waves from scattering of stellar-mass black holes in galactic nuclei. Mon Not R Astron Soc 395:2127-2146. https://doi.org/10.1111/j.1365-2966.2009. 14653.x. arXiv:0807.2638

Olejak A, Belczynski K, Ivanova N (2021) Impact of common envelope development criteria on the formation of LIGO/Virgo sources. Astron Astrophys 651:A100. https://doi.org/10.1051/0004-6361/ 202140520. arXiv:2102.05649 [astro-ph.HE]

Öpik E (1924) Statistical studies of double stars: on the distribution of relative luminosities and distances of double stars in the Harvard revised photometry north of declination -31 degrees. Publ Obs Astron Univ Tartu 25(6):1-167. http://muuseum.to.ee/Main/HTML/vol_xxv_-_1924.htm

Orosz JA, McClintock JE, Aufdenberg JP, Remillard RA, Reid MJ, Narayan R, Gou L (2011) The mass of the black hole in Cygnus X-1. Astrophys J 742(2):84. https://doi.org/10.1088/0004-637X/742/2/ 84. arXiv:1106.3689 [astro-ph.HE]

O’Shaughnessy R, Kim C (2010) Pulsar binary birthrates with spin-opening angle correlations. Astrophys J 715:230-241. https://doi.org/10.1088/0004-637X/715/1/230. arXiv:0908.2049 [astro-ph.GA]

O’Shaughnessy R, Kim C, Kalogera V, Belczynski K (2008) Constraining population synthesis models via empirical binary compact object merger and supernova rates. Astrophys J 672:479-488. https:// doi.org/10.1086/523620

O’Shaughnessy R, Kalogera V, Belczynski K (2010) Binary compact object coalescence rates: the role of elliptical galaxies. Astrophys J 716:615-633. https://doi.org/10.1088/0004-637X/716/1/615. arXiv: 0908.3635 [astro-ph.CO]

Paczyński B (1976) Common envelope binaries. In: Eggleton P, Mitton S, Whelan J (eds) Structure and evolution of close binary systems. IAU symposium, vol 73. D. Reidel, Dordrecht, p 75. https://doi. org/10.1017/S0074180900011864

Paczyński B, Rhoads JE (1993) Radio transients from gamma-ray bursters. Astrophys J Lett 418:L5. https://doi.org/10.1086/187102. arXiv:9307024 [astro-ph]

Palmese A, Conselice CJ (2021) GW190521 from the merger of ultradwarf galaxies. Phys Rev Lett 126(18):181103. https://doi.org/10.1103/PhysRevLett.126.181103. arXiv:2009.10688 [astro-ph.GA]

Pan Z, Yang H (2021) Formation rate of extreme mass ratio inspirals in active galactic nuclei. Phys Rev D 103(10):103018. https://doi.org/10.1103/PhysRevD.103.103018. arXiv:2101.09146 [astro-ph.HE]

Park D, Kim C, Lee HM, Bae YB, Belczynski K (2017) Black hole binaries dynamically formed in globular clusters. Mon Not R Astron Soc 469:4665-4674. https://doi.org/10.1093/mnras/stx1015. arXiv:1703.01568 [astro-ph.HE]

Pasham DR, Strohmayer TE, Mushotzky RF (2014) A 400-solar-mass black hole in the galaxy M82. Nature 513:74-76. https://doi.org/10.1038/nature13710

Paul D (2018) Binary neutron star merger rate via the luminosity function of short gamma-ray bursts. Mon Not R Astron Soc 477(4):4275-4284. https://doi.org/10.1093/mnras/sty840. arXiv:1710.05620 [astro-ph.HE]

Paynter J, Webster R, Thrane E (2021) Evidence for an intermediate-mass black hole from a gravitationally lensed gamma-ray burst. Nature Astron https://doi.org/10.1038/s41550-021-01307-1. arXiv:2103.15414 [astro-ph.HE]

Peng P, Chen X (2021) The last migration trap of compact objects in AGN accretion disc. Mon Not R Astron Soc 505(1):1324-1333. https://doi.org/10.1093/mnras/stab1419. arXiv:2104.07685 [astroph.HE]

Perets HB, Kratter KM (2012) The triple evolution dynamical instability: stellar collisions in the field and the formation of exotic binaries. Astrophys J 760:99. https://doi.org/10.1088/0004-637X/760/2/99. arXiv:1203.2914 [astro-ph.SR] 
Peters PC (1964) Gravitational radiation and the motion of two point masses. Phys Rev 136:1224-1232. https://doi.org/10.1103/PhysRev.136.B1224

Petrillo CE, Dietz A, Cavaglià M (2013) Compact object coalescence rate estimation from short gammaray burst observations. Astrophys J 767(2):140. https://doi.org/10.1088/0004-637X/767/2/140. arXiv:1202.0804 [astro-ph.CO]

Petrovich C, Antonini F (2017) Greatly enhanced merger rates of compact-object binaries in nonspherical nuclear star clusters. Astrophys J 846(2):146. https://doi.org/10.3847/1538-4357/aa8628. arXiv:1705.05848 [astro-ph.HE]

Pfahl E, Podsiadlowski P, Rappaport S (2005) Relativistic binary pulsars with black hole companions. Astrophys J 628:343-352. https://doi.org/10.1086/430515. arXiv:0502122

Phinney ES (1991) The rate of neutron star binary mergers in the universe: minimal predictions for gravity wave detector. Astrophys J 380:L17

Pleunis Z, Good DC, Kaspi VM, Mckinven R, Ransom SM, Scholz P, Bandura K, Bhardwaj M, Boyle PJ, Brar C, Cassanelli T, Chawla P, Fengqiu, Dong, Fonseca E, Gaensler BM, Josephy A, Kaczmarek JF, Leung C, Lin HH, Masui KW, Mena-Parra J, Michilli D, Ng C, Patel C, Rafiei-Ravandi M, Rahman M, Sanghavi P, Shin K, Smith KM, Stairs IH, Tendulkar SP (2021) Fast radio burst morphology in the first CHIME/FRB Catalog. Astrophys J 923:1. https://doi.org/10.3847/15384357/ac33ac. arXiv:2106.04356 [astro-ph.HE]

Podsiadlowski P, Langer N, Poelarends AJT, Rappaport S, Heger A, Pfahl E (2004) The effects of binary evolution on the dynamics of core collapse and neutron star kicks. Astrophys J 612(2):1044-1051. https://doi.org/10.1086/421713. arXiv:0309588 [astro-ph]

Pol N, McLaughlin M, Lorimer DR (2020) An updated galactic double neutron star merger rate based on radio pulsar populations. Res Notes AAS 4(2):22. https://doi.org/10.3847/2515-5172/ab7307. arXiv: 2002.10225 [astro-ph.HE]

Pol N, McLaughlin M, Lorimer D (2021) Insights into the Milky Way pulsar-black hole population using radio and gravitational wave observations. arXiv e-prints arXiv:2109.04512 [astro-ph.HE]

Portegies Zwart SF, McMillan SLW (2000) Black hole mergers in the universe. Astrophys J Lett 528(1):L17-L20. https://doi.org/10.1086/312422. arXiv:9910061 [astro-ph]

Postnov KA, Yungelson LR (2014) The evolution of compact binary star systems. Living Rev Relativ 17:3. https://doi.org/10.12942//rr-2014-3. arXiv:1403.4754 [astro-ph.HE]

Quinlan GD (1996) The dynamical evolution of massive black hole binaries I. Hardening in a fixed stellar background. New Astron 1:35-56. https://doi.org/10.1016/S1384-1076(96)00003-6. arXiv:9601092

Raidal M, Spethmann C, Vaskonen V, Veermäe H (2019) Formation and evolution of primordial black hole binaries in the early universe. J Cosmol Astropart Phys 2:018. https://doi.org/10.1088/14757516/2019/02/018. arXiv:1812.01930 [astro-ph.CO]

Rasskazov A, Kocsis B (2019) The rate of stellar mass black hole scattering in galactic nuclei. Astrophys J 881(1):20. https://doi.org/10.3847/1538-4357/ab2c74. arXiv:1902.03242 [astro-ph.HE]

Rastello S, Amaro-Seoane P, Arca-Sedda M, Capuzzo-Dolcetta R, Fragione G, Tosta e Melo I (2019) Stellar black hole binary mergers in open clusters. Mon Not R Astron Soc 483(1):1233-1246. https://doi.org/10.1093/mnras/sty3193. arXiv:1811.10628 [astro-ph.GA]

Rastello S, Mapelli M, Di Carlo UN, Giacobbo N, Santoliquido F, Spera M, Ballone A, Iorio G (2020) Dynamics of black hole-neutron star binaries in young star clusters. Mon Not R Astron Soc 497(2):1563-1570. https://doi.org/10.1093/mnras/staa2018. arXiv:2003.02277 [astro-ph.HE]

Rastello S, Mapelli M, Di Carlo UN, Iorio G, Ballone A, Giacobbo N, Santoliquido F, Torniamenti S (2021) Dynamics of binary black holes in low-mass young star clusters. Mon Not R Astron Soc 507(3):3612-3625. https://doi.org/10.1093/mnras/stab2355. arXiv:2105.01669 [astro-ph.GA]

Ravi V, Lasky PD (2014) The birth of black holes: neutron star collapse times, gamma-ray bursts and fast radio bursts. Mon Not R Astron Soc 441(3):2433-2439. https://doi.org/10.1093/mnras/stu720. arXiv:1403.6327 [astro-ph.HE]

Reitze D, Adhikari RX, Ballmer S, Barish B, Barsotti L, Billingsley G, Brown DA, Chen Y, Coyne D, Eisenstein R, Evans M, Fritschel P, Hall ED, Lazzarini A, Lovelace G, Read J, Sathyaprakash BS, Shoemaker D, Smith J, Torrie C, Vitale S, Weiss R, Wipf C, Zucker M (2019) Cosmic explorer: the U.S. contribution to gravitational-wave astronomy beyond LIGO. Bulletin AAS, 51(7):035. https:// baas.aas.org/pub/2020n7i035. arXiv:1907.04833 [astro-ph.IM]

Renzo M, Zapartas E, de Mink SE, Götberg Y, Justham S, Farmer RJ, Izzard RG, Toonen S, Sana H (2019) Massive runaway and walkaway stars. A study of the kinematical imprints of the physical processes governing the evolution and explosion of their binary progenitors. Astron Astrophys 624:A66. https://doi.org/10.1051/0004-6361/201833297. arXiv:1804.09164 [astro-ph.SR] 
Repetto S, Davies MB, Sigurdsson S (2012) Investigating stellar-mass black hole kicks. Mon Not R Astron Soc 425:2799-2809. https://doi.org/10.1111/j.1365-2966.2012.21549.x. arXiv:1203.3077 [astro-ph.GA]

Resmi L, Schulze S, Ishwara-Chandra CH, Misra K, Buchner J, De Pasquale M, Sánchez-Ramírez R, Klose S, Kim S, Tanvir NR, O'Brien PT (2018) Low-frequency view of GW170817/GRB 170817A with the giant metrewave radio telescope. Astrophys J 867(1):57. https://doi.org/10.3847/15384357/aae1a6. arXiv:1803.02768 [astro-ph.HE]

Ricker PM, Timmes FX, Taam RE, Webbink RF (2019) Common envelope evolution of massive stars. In: Oskinova LM, Bozzo E, Bulik T, Gies DR (eds) IAU Symposium. IAU symposium, vol 346, pp 449-454. https://doi.org/10.1017/S1743921318007433. arXiv:1811.03656 [astro-ph.SR]

Riley J, Mandel I, Marchant P, Butler E, Nathaniel K, Neijssel C, Shortt S, Vigna-Gómez A (2021) Chemically homogeneous evolution: a rapid population synthesis approach. Mon Not R Astron Soc 505(1):663-676. https://doi.org/10.1093/mnras/stab1291. arXiv:2010.00002 [astro-ph.SR]

Rodriguez CL, Antonini F (2018) A triple origin for the heavy and low-spin binary black holes detected by LIGO/VIRGO. Astrophys J 863(1):7. https://doi.org/10.3847/1538-4357/aacea4. arXiv:1805. 08212 [astro-ph.HE]

Rodriguez CL, Loeb A (2018) Redshift evolution of the black hole merger rate from globular clusters. Astrophys J Lett 866(1):L5. https://doi.org/10.3847/2041-8213/aae377. arXiv:1809.01152 [astroph.HE]

Rodriguez CL, Morscher M, Pattabiraman B, Chatterjee S, Haster CJ, Rasio FA (2015) Binary black hole mergers from globular clusters: implications for advanced LIGO. Phys Rev Lett 115(5):051101. https://doi.org/10.1103/PhysRevLett.115.051101. arXiv:1505.00792 [astro-ph.HE]

Rodriguez CL, Chatterjee S, Rasio FA (2016) Binary black hole mergers from globular clusters: masses, merger rates, and the impact of stellar evolution. Phys Rev D 93(8):084029. https://doi.org/10.1103/ PhysRevD.93.084029. arXiv:1602.02444 [astro-ph.HE]

Rodriguez CL, Zevin M, Pankow C, Kalogera V, Rasio FA (2016) Illuminating black hole binary formation channels with spins in advanced LIGO. Astrophys J Lett 832(1):L2. https://doi.org/10. 3847/2041-8205/832/1/L2. arXiv:1609.05916 [astro-ph.HE]

Rodriguez CL, Amaro-Seoane P, Chatterjee S, Rasio FA (2018) Post-Newtonian dynamics in dense star clusters: highly eccentric, highly spinning, and repeated binary black hole mergers. Phys Rev Lett 120(15):151101. https://doi.org/10.1103/PhysRevLett.120.151101. arXiv:1712.04937 [astro-ph.HE]

Román-Garza J, Bavera SS, Fragos T, Zapartas E, Misra D, Andrews J, Coughlin S, Dotter A, Kovlakas K, Serra JG, Qin Y, Rocha KA, Tran NH (2021) The role of core-collapse physics in the observability of black hole neutron star mergers as multimessenger sources. Astrophys $\mathrm{J}$ Lett 912(2):L23. https://doi.org/10.3847/2041-8213/abf42c. arXiv:2012.02274 [astro-ph.HE]

Romero-Shaw I, Lasky PD, Thrane E (2021) Signs of eccentricity in two gravitational-wave signals may indicate a subpopulation of dynamically assembled binary black holes. Astrophys J Lett 921(2):L31. https://doi.org/10.3847/2041-8213/ac3138. arXiv:2108.01284 [astro-ph.HE]

Romero-Shaw IM, Farrow N, Stevenson S, Thrane E, Zhu XJ (2020) On the origin of GW190425. Mon Not R Astron Soc 496(1):L64-L69. https://doi.org/10.1093/mnrasl/slaa084. arXiv:2001.06492 [astro-ph.HE]

Rose SC, Naoz S, Geller AM (2019) Companion-driven evolution of massive stellar binaries. Mon Not R Astron Soc 488(2):2480-2492. https://doi.org/10.1093/mnras/stz1846. arXiv:1903.12185 [astroph.SR]

Rosswog S, Ramirez-Ruiz E (2002) Jets, winds and bursts from coalescing neutron stars. Mon Not R Astron Soc 336(1):L7-L11. https://doi.org/10.1046/j.1365-8711.2002.05898.x. arXiv:0207576 [astro-ph]

Rosswog S, Liebendörfer M, Thielemann FK, Davies MB, Benz W, Piran T (1999) Mass ejection in neutron star mergers. Astron Astrophys 341:499-526 arXiv:9811367 [astro-ph]

Roulet J, Venumadhav T, Zackay B, Dai L, Zaldarriaga M (2020) Binary black hole mergers from LIGO/ Virgo $\mathrm{O} 1$ and O2: Population inference combining confident and marginal events. Phys Rev D 102(12):123022. https://doi.org/10.1103/PhysRevD.102.123022. arXiv:2008.07014 [astro-ph.HE]

Roulet J, Chia HS, Olsen S, Dai L, Venumadhav T, Zackay B, Zaldarriaga M (2021) Distribution of effective spins and masses of binary black holes from the LIGO and Virgo O1-O3a observing runs. Phys Rev D 104(8):083010. https://doi.org/10.1103/PhysRevD.104.083010. arXiv:2105.10580 [astro-ph.HE] 
Sakstein J, Croon D, McDermott SD, Straight MC, Baxter EJ (2020) Beyond the standard model explanations of GW190521. Phys Rev Lett 125(26):261105. https://doi.org/10.1103/PhysRevLett. 125.261105. arXiv:2009.01213 [gr-qc]

Samsing J, MacLeod M, Ramirez-Ruiz E (2014) The formation of eccentric compact binary inspirals and the role of gravitational wave emission in binary-single stellar encounters. Astrophys J 784:71. https://doi.org/10.1088/0004-637X/784/1/71. arXiv:1308.2964 [astro-ph.HE]

Sana H, de Mink SE, de Koter A, Langer N, Evans CJ, Gieles M, Gosset E, Izzard RG, Le Bouquin JB, Schneider FRN (2012) Binary interaction dominates the evolution of massive stars. Science 337:444. https://doi.org/10.1126/science.1223344. arXiv:1207.6397 [astro-ph.SR]

Santoliquido F, Mapelli M, Bouffanais Y, Giacobbo N, Di Carlo UN, Rastello S, Artale MC, Ballone A (2020) The cosmic merger rate density evolution of compact binaries formed in young star clusters and in isolated binaries. Astrophys J 898(2):152. https://doi.org/10.3847/1538-4357/ab9b78. arXiv: 2004.09533 [astro-ph.HE]

Santoliquido F, Mapelli M, Giacobbo N, Bouffanais Y, Artale MC (2021) The cosmic merger rate density of compact objects: impact of star formation, metallicity, initial mass function, and binary evolution. Mon Not R Astron Soc 502(4):4877-4889. https://doi.org/10.1093/mnras/stab280. arXiv:2009. 03911 [astro-ph.HE]

Schneider FRN, Podsiadlowski P, Müller B (2021) Pre-supernova evolution, compact-object masses, and explosion properties of stripped binary stars. Astron Astrophys 645:A5. https://doi.org/10.1051/ 0004-6361/202039219. arXiv:2008.08599 [astro-ph.SR]

Secunda A, Bellovary J, Mac Low MM, Ford KES, McKernan B, Leigh NWC, Lyra W, Sándor Z (2019) Orbital migration of interacting stellar mass black holes in disks around supermassive black holes. Astrophys J 878(2):85. https://doi.org/10.3847/1538-4357/ab20ca. arXiv:1807.02859 [astro-ph.HE]

Secunda A, Bellovary J, Mac Low MM, Ford KES, McKernan B, Leigh NWC, Lyra W, Sándor Z, Adorno JI (2020) Orbital migration of interacting stellar mass black holes in disks around supermassive black holes. II. Spins and incoming objects. Astrophys J 903(2):133. https://doi.org/ 10.3847/1538-4357/abbc1d. arXiv:2004.11936 [astro-ph.HE]

Sepinsky JF, Willems B, Kalogera V, Rasio FA (2010) Interacting binaries with eccentric orbits. III. Orbital evolution due to direct impact and self-accretion. Astrophys J 724(1):546-558. https://doi. org/10.1088/0004-637X/724/1/546. arXiv:1005.0625 [astro-ph.SR]

Shao Y, Li XD (2021) Population synthesis of black hole binaries with compact star companions. Astrophys J 920:81. https://doi.org/10.3847/1538-4357/ac173e. arXiv:2107.03565 [astro-ph.HE]

Shappee BJ, Thompson TA (2013) The mass-loss-induced eccentric Kozai mechanism: a new channel for the production of close compact object-stellar binaries. Astrophys J 766(1):64. https://doi.org/10. 1088/0004-637X/766/1/64. arXiv:1204.1053 [astro-ph.SR]

Siegel DM, Barnes J, Metzger BD (2019) Collapsars as a major source of r-process elements. Nature 569(7755):241-244. https://doi.org/10.1038/s41586-019-1136-0. arXiv:1810.00098 [astro-ph.HE]

Sigurdsson S, Hernquist L (1993) Primordial black holes in globular clusters. Nature 364:423-425. https://doi.org/10.1038/364423a0

Sigurdsson S, Phinney ES (1993) Binary-single star interactions in globular clusters. Astrophys J 415:631. https://doi.org/10.1086/173190

Silsbee K, Tremaine S (2017) Lidov-Kozai cycles with gravitational radiation: merging black holes in isolated triple systems. Astrophys J 836:39. https://doi.org/10.3847/1538-4357/aa5729. arXiv:1608. 07642 [astro-ph.HE]

Sipior MS, Sigurdsson S (2002) Nova Scorpii and coalescing low-mass black hole binaries as LIGO sources. Astrophys J 572(2):962-970. https://doi.org/10.1086/340370. arXiv:0202385 [astro-ph]

Smarr LL, Blandford R (1976) The binary pulsar-physical processes, possible companions, and evolutionary histories. Astrophys J 207:574-588. https://doi.org/10.1086/154524

Smartt SJ, Chen TW, Jerkstrand A, Coughlin M, Kankare E, Sim SA, Fraser M, Inserra C, Maguire K, Chambers KC et al (2017) A kilonova as the electromagnetic counterpart to a gravitational-wave source. Nature 551(7678):75-79. https://doi.org/10.1038/nature24303. arXiv:1710.05841 [astroph.HE]

Soberman GE, Phinney ES, van den Heuvel EPJ (1997) Stability criteria for mass transfer in binary stellar evolution. Astron Astrophys 327:620-635 arXiv:9703016 [astro-ph]

Spera M, Mapelli M, Giacobbo N, Trani AA, Bressan A, Costa G (2019) Merging black hole binaries with the SEVN code. Mon Not R Astron Soc 485(1):889-907. https://doi.org/10.1093/mnras/stz359. arXiv:1809.04605 [astro-ph.HE] 
Spitzer L Jr (1969) Equipartition and the formation of compact nuclei in spherical stellar systems. Astrophys J Lett 158:L139. https://doi.org/10.1086/180451

Stacy A, Bromm V, Lee AT (2016) Building up the Population III initial mass function from cosmological initial conditions. Mon Not R Astron Soc 462(2):1307-1328. https://doi.org/10.1093/ mnras/stw1728. arXiv:1603.09475 [astro-ph.GA]

Stephan AP, Naoz S, Ghez AM, Witzel G, Sitarski BN, Do T, Kocsis B (2016) Merging binaries in the Galactic Center: the eccentric Kozai-Lidov mechanism with stellar evolution. Mon Not R Astron Soc 460(4):3494-3504. https://doi.org/10.1093/mnras/stw1220. arXiv:1603.02709 [astro-ph.SR]

Stephan AP, Naoz S, Ghez AM, Morris MR, Ciurlo A, Do T, Breivik K, Coughlin S, Rodriguez CL (2019) The fate of binaries in the galactic center: the mundane and the exotic. Astrophys $\mathrm{J}$ 878(1):58. https://doi.org/10.3847/1538-4357/ab1e4d. arXiv:1903.00010 [astro-ph.SR]

Stevenson S, Ohme F, Fairhurst S (2015) Distinguishing compact binary population synthesis models using gravitational wave observations of coalescing binary black holes. Astrophys J 810:58. https:// doi.org/10.1088/0004-637X/810/1/58. arXiv:1504.07802 [astro-ph.HE]

Stevenson S, Sampson M, Powell J, Vigna-Gómez A, Neijssel CJ, Szécsi D, Mandel I (2019) The impact of pair-instability mass loss on the binary black hole mass distribution. Astrophys J 882(2):121. https://doi.org/10.3847/1538-4357/ab3981. arXiv:1904.02821 [astro-ph.HE]

Stone NC, Metzger BD, Haiman Z (2017) Assisted inspirals of stellar mass black holes embedded in AGN discs: solving the final au problem. Mon Not R Astron Soc 464:946-954. https://doi.org/10. 1093/mnras/stw2260. arXiv:1602.04226

Sweet PA (1950) The importance of rotation in stellar evolution. Mon Not R Astron Soc 110:548. https:// doi.org/10.1093/mnras/110.6.548

Szölgyén Á, Kocsis B (2018) Black hole disks in galactic nuclei. Phys Rev Lett 121(10):101101. https:// doi.org/10.1103/PhysRevLett.121.101101. arXiv:1803.07090 [astro-ph.GA]

Tagawa H, Haiman Z, Kocsis B (2020) Formation and evolution of compact-object binaries in AGN disks. Astrophys J 898(1):25. https://doi.org/10.3847/1538-4357/ab9b8c. arXiv:1912.08218 [astroph.GA]

Tagawa H, Kocsis B, Haiman Z, Bartos I, Omukai K, Samsing J (2021) Mass-gap mergers in active galactic nuclei. Astrophys J 908(2):194. https://doi.org/10.3847/1538-4357/abd555. arXiv:2012. 00011 [astro-ph.HE]

Tang PN, Eldridge JJ, Stanway ER, Bray JC (2020) Dependence of gravitational wave transient rates on cosmic star formation and metallicity evolution history. Mon Not R Astron Soc 493(1):L6-L10. https://doi.org/10.1093/mnrasl/slz183. arXiv:1912.04474 [astro-ph.GA]

Tanikawa A, Susa H, Yoshida T, Trani AA, Kinugawa T (2021) Merger rate density of population III binary black holes below, above, and in the pair-instability mass gap. Astrophys J 910(1):30. https:// doi.org/10.3847/1538-4357/abe40d. arXiv:2008.01890 [astro-ph.HE]

Tanvir NR, Levan AJ, Fruchter AS, Hjorth J, Hounsell RA, Wiersema K, Tunnicliffe RL (2013) A kilonova associated with the short-duration $\gamma$-ray burst GRB130603B. Nature 500:547-549. https:// doi.org/10.1038/nature12505. arXiv:1306.4971 [astro-ph.HE]

Tauris TM, Langer N, Podsiadlowski P (2015) Ultra-stripped supernovae: progenitors and fate. Mon Not R Astron Soc 451:2123-2144. https://doi.org/10.1093/mnras/stv990. arXiv:1505.00270 [astroph.SR]

Tauris TM, Kramer M, Freire PCC, Wex N, Janka HT, Langer N, Podsiadlowski P, Bozzo E, Chaty S, Kruckow MU, van den Heuvel EPJ, Antoniadis J, Breton RP, Champion DJ (2017) Formation of double neutron star systems. Astrophys J 846:170. https://doi.org/10.3847/1538-4357/aa7e89. arXiv: 1706.09438 [astro-ph.HE]

Taylor M, Cinabro D, Dilday B, Galbany L, Gupta RR, Kessler R, Marriner J, Nichol RC, Richmond M, Schneider DP, Sollerman J (2014) The core collapse supernova rate from the SDSS-II supernova survey. Astrophys J 792(2):135. https://doi.org/10.1088/0004-637X/792/2/135. arXiv:1407.0999 [astro-ph.SR]

Team COMPAS: Riley J, Agrawal P, Barrett JW, Boyett KNK, Broekgaarden FS, Chattopadhyay D, Gaebel SM, Gittins F, Hirai R, Howitt G, Justham S, Khandelwal L, Kummer F, Lau MYM, Mandel I, de Mink SE, Neijssel C, Riley T, van Son L, Stevenson S, Vigna-Gomez A, Vinciguerra S, Wagg T, Willcox R (2021) Rapid stellar and binary population synthesis with COMPAS. arXiv e-prints arXiv:2109.10352 [astro-ph.IM]

Toonen S, Portegies Zwart S, Hamers AS, Bandopadhyay D (2020) The evolution of stellar triples. The most common evolutionary pathways. Astron Astrophys 640:A16. https://doi.org/10.1051/00046361/201936835. arXiv:2004.07848 [astro-ph.SR] 
Totani T (2013) Cosmological fast radio bursts from binary neutron star mergers. Publ Astron Soc Japan 65:L12. https://doi.org/10.1093/pasj/65.5.L12. arXiv:1307.4985 [astro-ph.HE]

Trani AA, Rastello S, Di Carlo UN, Santoliquido F, Tanikawa A, Mapelli M (2021) Compact object mergers in hierarchical triples from low-mass young star clusters. arXiv e-prints arXiv:2111.06388 [astro-ph.HE]

Troja E, King AR, O'Brien PT, Lyons N, Cusumano G (2008) Different progenitors of short hard gammaray bursts. Mon Not R Astron Soc 385(1):L10-L14. https://doi.org/10.1111/j.1745-3933.2007. 00421.x. arXiv:0711.3034 [astro-ph]

Troja E, Piro L, van Eerten H, Wollaeger RT, Im M, Fox OD, Butler NR, Cenko SB, Sakamoto T, Fryer CL, Ricci R, Lien A, Ryan RE, Korobkin O, Lee SK, Burgess JM, Lee WH, Watson AM, Choi C, Covino S, D’Avanzo P, Fontes CJ, González JB, Khandrika HG, Kim J, Kim SL, Lee CU, Lee HM, Kutyrev A, Lim G, Sánchez-Ramírez R, Veilleux S, Wieringa MH, Yoon Y (2017) The X-ray counterpart to the gravitational-wave event GW170817. Nature 551:71-74. https://doi.org/10.1038/ nature24290. arXiv:1710.05433 [astro-ph.HE]

Troja E, Piro L, Ryan G, van Eerten H, Ricci R, Wieringa MH, Lotti S, Sakamoto T, Cenko SB (2018) The outflow structure of GW170817 from late-time broad-band observations. Mon Not R Astron Soc 478(1):L18-L23. https://doi.org/10.1093/mnrasl/sly061. arXiv:1801.06516 [astro-ph.HE]

Tsang D (2013) Shattering flares during close encounters of neutron stars. Astrophys J 777:103. https:// doi.org/10.1088/0004-637X/777/2/103. arXiv:1307.3554 [astro-ph.HE]

Tutukov A, Yungelson L (1973) Evolution of massive close binaries. Nauchn Inform 27:70

Tutukov AV, Yungelson LR (1993) The merger rate of neutron star and black hole binaries. Mon Not R Astron Soc 260:675-678. https://doi.org/10.1093/mnras/260.3.675

van den Heuvel EPJ (1976) Late stages of close binary systems. In: Eggleton P, Mitton S, Whelan J (eds) Structure and evolution of close binary systems. IAU symposium, vol 73. D. Reidel, Dordrecht, pp 35-61. https://doi.org/10.1017/S0074180900011839

van den Heuvel EPJ, De Loore C (1973) The nature of X-ray binaries III. Evolution of massive close binaries with one collapsed component - with a possible application to Cygnus X-3. Astron Astrophys 25:387-395

van den Heuvel EPJ, Portegies Zwart SF, de Mink SE (2017) Forming short-period Wolf-Rayet X-ray binaries and double black holes through stable mass transfer. Mon Not $\mathrm{R}$ Astron Soc 471:4256-4264. https://doi.org/10.1093/mnras/stx1430. arXiv:1701.02355 [astro-ph.SR]

van Son LAC, De Mink SE, Broekgaarden FS, Renzo M, Justham S, Laplace E, Morán-Fraile J, Hendriks DD, Farmer R (2020) Polluting the pair-instability mass gap for binary black holes through supereddington accretion in isolated binaries. Astrophys J 897(1):100. https://doi.org/10.3847/1538-4357/ ab9809. arXiv:2004.05187 [astro-ph.HE]

van Son LAC, de Mink SE, Callister T, Justham S, Renzo M, Wagg T, Broekgaarden FS, Kummer F, Pakmor R, Mandel I (2021) The redshift evolution of the binary black hole merger rate: a weighty matter. arXiv e-prints arXiv:2110.01634 [astro-ph.HE]

Veitch J, Pürrer M, Mandel I (2015) Measuring intermediate-mass black-hole binaries with advanced gravitational wave detectors. Phys Rev Lett 115(14):141101. https://doi.org/10.1103/PhysRevLett. 115.141101. arXiv:1503.05953 [astro-ph.HE]

Venumadhav T, Zackay B, Roulet J, Dai L, Zaldarriaga M (2020) New binary black hole mergers in the second observing run of advanced LIGO and advanced virgo. Phys Rev D 101(8):083030. https:// doi.org/10.1103/PhysRevD.101.083030. arXiv:1904.07214 [astro-ph.HE]

Verbunt F, Igoshev A, Cator E (2017) The observed velocity distribution of young pulsars. Astron Astrophys 608:A57. https://doi.org/10.1051/0004-6361/201731518. arXiv:1708.08281 [astroph.HE]

Vesperini E, McMillan SLW, D'Ercole A, D'Antona F (2010) Intermediate-mass black holes in early globular clusters. Astrophys J Lett 713:L41-L44. https://doi.org/10.1088/2041-8205/713/1/L41. arXiv:1003.3470 [astro-ph.GA]

Vick M, Lai D (2018) Dynamical tides in highly eccentric binaries: chaos, dissipation, and quasi-steady state. Mon Not R Astron Soc 476(1):482-495. https://doi.org/10.1093/mnras/sty225. arXiv:1708. 09392 [astro-ph.SR]

Vigna-Gómez A, Neijssel CJ, Stevenson S, Barrett JW, Belczynski K, Justham S, de Mink SE, Müller B, Podsiadlowski P, Renzo M, Szécsi D, Mandel I (2018) On the formation history of Galactic double neutron stars. Mon Not R Astron Soc 481:4009-4029. https://doi.org/10.1093/mnras/sty2463. arXiv: 1805.07974 [astro-ph.SR] 
Vigna-Gómez A, Schrøder SL, Ramirez-Ruiz E, Aguilera-Dena DR, Batta A, Langer N, Wilcox R (2021a) Fallback supernova assembly of heavy binary neutron stars and light black hole-neutron star pairs and the common stellar ancestry of GW190425 and GW200115. Astrophys J Lett 920:L17. https://doi.org/10.3847/2041-8213/ac2903. arXiv:2106.12381 [astro-ph.HE]

Vigna-Gómez A, Toonen S, Ramirez-Ruiz E, Leigh NWC, Riley J, Haster CJ (2021) Massive stellar triples leading to sequential binary black hole mergers in the field. Astrophys J Lett 907(1):L19. https://doi.org/10.3847/2041-8213/abd5b7. arXiv:2010.13669 [astro-ph.HE]

Vinciguerra S, Neijssel CJ, Vigna-Gómez A, Mandel I, Podsiadlowski P, Maccarone TJ, Nicholl M, Kingdon S, Perry A, Salemi F (2020) Be X-ray binaries in the SMC as indicators of mass-transfer efficiency. Mon Not R Astron Soc 498(4):4705-4720. https://doi.org/10.1093/mnras/staa2177. arXiv:2003.00195 [astro-ph.HE]

Vink JS (2017) Mass loss and stellar superwinds. Philos Trans R Soc Lond Ser A 375(2105):20160269. https://doi.org/10.1098/rsta.2016.0269. arXiv:1610.00578 [astro-ph.SR]

Volonteri M (2010) Formation of supermassive black holes. Astron Astrophys Rev 18:279-315. https:// doi.org/10.1007/s00159-010-0029-x. arXiv:1003.4404

Voss R, Tauris TM (2003) Galactic distribution of merging neutron stars and black holes - prospects for short gamma-ray burst progenitors and LIGO/VIRGO. Mon Not R Astron Soc 342(4):1169-1184. https://doi.org/10.1046/j.1365-8711.2003.06616.x. arXiv:0303227 [astro-ph]

Vynatheya P, Hamers AS (2021) How important is secular evolution for black hole and neutron star mergers in 2+2 and 3+1 quadruple-star systems? arXiv e-prints arXiv:2110.14680 [astro-ph.HE]

Wang H, Stephan AP, Naoz S, Hoang BM, Breivik K (2021) Gravitational-wave signatures from compact object binaries in the galactic center. Astrophys J 917(2):76. https://doi.org/10.3847/1538-4357/ ac088d. arXiv:2010.15841 [astro-ph.HE]

Webbink RF (1984) Double white dwarfs as progenitors of R Coronae Borealis stars and Type I supernovae. Astrophys J 277:355-360. https://doi.org/10.1086/161701

Wen L (2003) On the eccentricity distribution of coalescing black hole binaries driven by the Kozai mechanism in globular clusters. Astrophys J 598:419-430. https://doi.org/10.1086/378794. arXiv: 0211492 [astro-ph]

Wong KWK, Breivik K, Kremer K, Callister T (2021) Joint constraints on the field-cluster mixing fraction, common envelope efficiency, and globular cluster radii from a population of binary hole mergers via deep learning. Phys Rev D 103(8):083021. https://doi.org/10.1103/PhysRevD.103. 083021. arXiv:2011.03564 [astro-ph.HE]

Wysocki D, Gerosa D, O’Shaughnessy R, Belczynski K, Gladysz W, Berti E, Kesden M, Holz DE (2018) Explaining LIGO's observations via isolated binary evolution with natal kicks. Phys Rev D 97(4):043014. https://doi.org/10.1103/PhysRevD.97.043014. arXiv:1709.01943 [astro-ph.HE]

Yang S, Valenti S, Cappellaro E, Sand DJ, Tartaglia L, Corsi A, Reichart DE, Haislip J, Kouprianov V (2017) An empirical limit on the kilonova rate from the DLT40 one day cadence supernova survey. Astrophys J Lett 851(2):L48. https://doi.org/10.3847/2041-8213/aaa07d. arXiv:1710.05864 [astroph.HE]

Yang Y, Bartos I, Gayathri V, Ford KES, Haiman Z, Klimenko S, Kocsis B, Márka S, Márka Z, McKernan B, O’Shaughnessy R (2019) Hierarchical black hole mergers in active galactic nuclei. Phys Rev Lett 123(18):181101. https://doi.org/10.1103/PhysRevLett.123.181101. arXiv:1906.09281 [astro-ph.HE]

Yang Y, Bartos I, Haiman Z, Kocsis B, Márka S, Tagawa H (2020) Cosmic evolution of stellar-mass black hole merger rate in active galactic nuclei. Astrophys J 896(2):138. https://doi.org/10.3847/ 1538-4357/ab91b4. arXiv:2003.08564 [astro-ph.HE]

Ye CS, Fong Wf, Kremer K, Rodriguez CL, Chatterjee S, Fragione G, Rasio FA (2020) On the rate of neutron star binary mergers from globular clusters. Astrophys J Lett 888(1):L10. https://doi.org/10. 3847/2041-8213/ab5dc5. arXiv:1910.10740 [astro-ph.HE]

Yoon SC, Langer N, Norman C (2006) Single star progenitors of long gamma-ray bursts. I. Model grids and redshift dependent GRB rate. Astron Astrophys 460:199-208. https://doi.org/10.1051/00046361:20065912. arXiv:0606637 [astro-ph]

Zackay B, Venumadhav T, Dai L, Roulet J, Zaldarriaga M (2019) Highly spinning and aligned binary black hole merger in the Advanced LIGO first observing run. Phys Rev D 100(2):023007. https:// doi.org/10.1103/PhysRevD.100.023007. arXiv:1902.10331 [astro-ph.HE]

Zahn JP (1977) Tidal friction in close binary stars. Astron Astrophys 57:383-394 
Zevin M, Spera M, Berry CPL, Kalogera V (2020) Exploring the lower mass gap and unequal mass regime in compact binary evolution. Astrophys J Lett 899(1):L1. https://doi.org/10.3847/2041-8213/ aba74e. arXiv:2006.14573 [astro-ph.HE]

Zevin M, Romero-Shaw IM, Kremer K, Thrane E, Lasky PD (2021) Implications of eccentric observations on binary black hole formation channels. Astrophys J Lett 921:L43. https://doi.org/10. 3847/2041-8213/ac32dc. arXiv:2106.09042 [astro-ph.HE]

Zhang B (2020) Fast radio bursts from interacting binary neutron star systems. Astrophys J Lett 890(2):L24. https://doi.org/10.3847/2041-8213/ab7244. arXiv:2002.00335 [astro-ph.HE]

Zhang BB, Zhang B, Sun H, Lei WH, Gao H, Li Y, Shao L, Zhao Y, Hu YD, Lü HJ, Wu XF, Fan XL, Wang G, Castro-Tirado AJ, Zhang S, Yu BY, Cao YY, Liang EW (2018) A peculiar low-luminosity short gamma-ray burst from a double neutron star merger progenitor. Nature Commun 9:447. https:// doi.org/10.1038/s41467-018-02847-3. arXiv:1710.05851 [astro-ph.HE]

Ziosi BM, Mapelli M, Branchesi M, Tormen G (2014) Dynamics of stellar black holes in young star clusters with different metallicities - II. Black hole-black hole binaries. Mon Not R Astron Soc 441:3703-3717. https://doi.org/10.1093/mnras/stu824. arXiv:1404.7147

Publisher's Note Springer Nature remains neutral with regard to jurisdictional claims in published maps and institutional affiliations. 